\title{
Distribution and efficacy of chemotherapeutics in the treatment of preclinical brain metastases of breast cancer
}

Tori Terrell Hall

Follow this and additional works at: https://researchrepository.wvu.edu/etd

\section{Recommended Citation}

Hall, Tori Terrell, "Distribution and efficacy of chemotherapeutics in the treatment of preclinical brain metastases of breast cancer" (2016). Graduate Theses, Dissertations, and Problem Reports. 5739. https://researchrepository.wvu.edu/etd/5739

This Dissertation is protected by copyright and/or related rights. It has been brought to you by the The Research Repository @ WVU with permission from the rights-holder(s). You are free to use this Dissertation in any way that is permitted by the copyright and related rights legislation that applies to your use. For other uses you must obtain permission from the rights-holder(s) directly, unless additional rights are indicated by a Creative Commons license in the record and/ or on the work itself. This Dissertation has been accepted for inclusion in WVU Graduate Theses, Dissertations, and Problem Reports collection by an authorized administrator of The Research Repository @ WVU.

For more information, please contact researchrepository@mail.wvu.edu. 


\title{
DISTRIBUTION AND EFFICACY OF CHEMOTHERAPEUTICS IN THE TREATMENT OF PRECLINICAL BRAIN METASTASES OF BREAST CANCER
}

\author{
TORI TERRELL HALL, B.S.
}

\author{
Dissertation submitted to the School of Pharmacy \\ at West Virginia University \\ in partial fulfillment of the requirements \\ for the degree of
Doctor of Philosophy
in \\ Pharmaceutical and Pharmacological Sciences
}

\author{
Paul R. Lockman, Ph.D., Committee Chairperson \\ Patrick Callery, Ph.D. \\ William Petros, Pharm.D. \\ Jason D. Huber, Ph.D. \\ Taura L. Barr, RN, Ph.D. \\ Department of Pharmaceutical Sciences \\ Morgantown, West Virginia \\ 2016
}

Keywords: blood-brain barrier, blood-tumor barrier, microfluidic device, in vitro, in vivo

Copyright 2016 Tori Terrell Hall 
ProQuest Number: 10246949

All rights reserved

INFORMATION TO ALL USERS

The quality of this reproduction is dependent upon the quality of the copy submitted.

In the unlikely event that the author did not send a complete manuscript and there are missing pages, these will be noted. Also, if material had to be removed, a note will indicate the deletion.

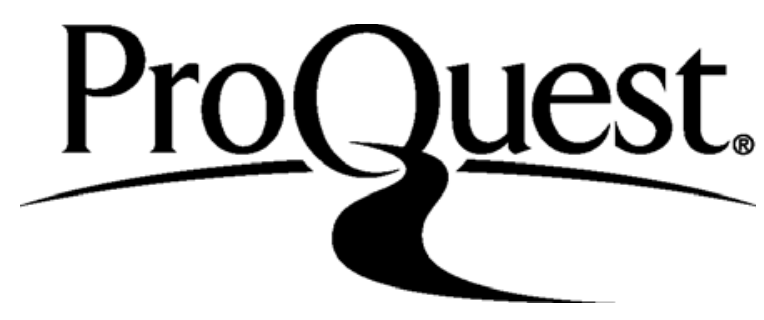

ProQuest 10246949

Published by ProQuest LLC (2016). Copyright of the Dissertation is held by the Author.

All rights reserved.

This work is protected against unauthorized copying under Title 17, United States Code Microform Edition @) ProQuest LLC.

ProQuest LLC.

789 East Eisenhower Parkway

P.O. Box 1346

Ann Arbor, Ml 48106 - 1346 


\section{ABSTRACT \\ DISTRIBUTION AND EFFICACY OF CHEMOTHERAPEUTICS IN THE TREATMENT OF PRECLINICAL BRAIN METASTASES OF BREAST CANCER}

\section{Tori Terrell Hall}

Brain metastases are a critical, life-threatening problem for women with advanced metastatic breast cancer. Approximately $80 \%$ of women with disseminated central lesions are unable to survive the first year after diagnosis. Despite the breakdown of the bloodbrain barrier, chemotherapeutics have limited penetration and distribution into brain metastases and are unable to induce cytotoxicity in the tumor. Limiting the development of new treatments for brain metastases of breast cancer, there are no commercially available in vitro models available that accurately model, and mimic the functionality of, the in vivo blood-tumor barrier (BTB). In an attempt to address the aforementioned problem, the following connected, but independent aims were proposed and completed in a novel microfluidic device: (1) Determine the permeability of three passive markers and one subject to efflux, in blood-brain barrier (BBB) and BTB models (2) Determine if trastuzumab crosses the $\mathrm{BBB}$ and $\mathrm{BTB}$ barrier in both in vivo and in vitro models (3) Evaluate if the microfluidic BBB and BTB models are relevant and comparable to current in vivo models. Further, based on the data presented herein, additional questions and trials have evolved into an evolution of the current microfluidic chip, discussed in the final chapter. This dissertation incorporates multiple innovative and complex experiments, which suggest that the current microfluidic chip accurately portrays the BBB and BTB when compared to the in vivo barriers, and is a readily available and rapid throughput model for all cancer, as well as BBB, researchers. 


\section{Dedication}

I would like to dedicate this work to Roxie Mae, Jaxson Don, Amelia Jo, and our future little(s) that have yet to arrive. I entered graduate school to become the best version of me I could be, and you guys push me towards that every single day. You are the sunshine of my life, and the joys of my heart. I love each one of you more than words could ever express, and I thank God for bestowing upon me the honor of being your momma. 


\section{Acknowledgements}

First and foremost, I would like to sincerely thank my mentor and advisor, Dr. Paul Lockman. Dr. Lockman, I am so thankful for the risk you took on me when you offered me a position in your lab back in 2012 . The ability to work with you over the last five years has been such an incredible honor, and advanced my scientific career in insurmountable ways. I want to thank you for allowing me to work on such a dynamic and unique project, and for not allowing me to give up in the summer of 2015, when I was pregnant with the twins and completely burnt out. The wisdom, mentorship, guidance, and inspiration you provided to me on a daily basis allowed me the room to improve and discover universal truths, as well as helped establish a solid foundation for my professional and scientific development. Thank you for the countless times you allowed me to sit in your office and cry and always making time to listen with your roll of paper towels tucked away. I know I would not have been able to navigate graduate school, each of my projects, two cross country moves, two pregnancies, three kids, and establishing (and maintaining) my family, without the unyielding tenderness, support, understanding and unprecedented patience you provided me over the last five years. The relationship I have with you and Julie is one that I will forever cherish, and I look forward to the continuation of that friendship as I take the next step of this journey.

Next I would like to acknowledge and thank each of my committee members for their willingness to be a part of my committee and for their contribution to my experiences throughout graduate school. Each of you have provided influential suggestions that have helped guide each of the projects included in this dissertation, as 
well as my overall progression as a professional. Dr. Petros, thank you all for the challenging questions, numerous discussions regarding translational research, and how each of my projects would successfully translate to the clinic. The passion you exert for patients has truly inspired me, and the support and faith you have invested within me has encouraged me to uphold excellence in every aspect of my life and career. Dr. Callery, thank you for providing me with such a strong platform of encouragement, and for the many discussions we had in your office about school, work, life and such; there is no doubt that your support has been a significant aid in my time at WVU. Dr. Huber, thank you for your valuable contributions and suggestions regarding the blood-brain barrier and permeability; your knowledge has undoubtedly helped form and aid in this dissertation. Dr. Barr, thank you for your support, encouragement, open door, and willing availability to sit and talk with me, regardless of the topic; your wisdom, knowledge, and guidance on navigating motherhood, work, and happiness has been an invaluable source throughout the last two years and I truly appreciate your insight.

To my lab mates, Dr. Chris Adkins, Afroz Mohammad, and Neal Shah, there is no doubt that I have gained immense knowledge from each of you and your willingness to aid in different parts of these dissertation. Chris, thank you for accepting me into the lab (almost) 5 years ago. I could not have asked for a better senior graduate student to have learned techniques, and lab demeanor from. You set an excellent example for Afroz and I, and I hope to have passed along the foundation of the lab in such a way that benefits Dr. Lockman to the max, as you did to me. Afroz, I will always cherish the last 4 years and the friendship we share. Thank you for always being willing to drop whatever you 
were doing at a moment's notice, to come and assist in whatever I was doing, or needing help with (even if it meant you and Neal staying up in the lab until 11 PM with me doing ENDLESS serial dilutions of radiation!!). You are the epitome of what one would hope to have in a lab mate and, most of all, a friend. You will always have a place to stay in Texas $:$ Neal, thank you for your sense of humor, your desire to learn, and your camaraderie. You were alongside Afroz and I in many of the crazy things we endured (at least for the last year and a half or so) and I thank you for your organization, functionality, and crazy taste in music. It definitely made those long hours in the IVIS more enjoyable. Thank you all for truly making the lab, and West Virginia, feel like family.

I would also like to thank Dr. Mohamed Nounou for his immeasurable guidance, the foundation of my my cell culture knowledge, and the countless phone calls and emails helping me solve whatever technical issue I may have been having. Nounou, thank you and Fatema, for your aid in the trastuzumab project. You will always be a part of my family. To Jessica Griffith and Emma Dolan, thank you both for your hard work and your assistance throughout your time in the lab. The contributions you both made have impacted my work, and my life, in more ways than one. To Dr. Julie Lockman, thank you for the fantastic job you did at teaching pharmacology at WTAMU. Had I not taken that course with you, I truly don't believe I would be where I am today. Thank you for your passion for teaching, for loving me (and my kids) as fiercely as you have over the last 8 years, and for the many lunch dates we had leading up to my joining Dr. Lockman's lab. Your friendship helped me through a really rough time in my life, and I sincerely thank 
you for that. To Dr. Karen Martin, Dr. Amanda Ammer, Sarah McLaughlin, and Emily Ellis, thank you for the morning coffee talks, the uncountable amounts of belly laughs, and for the unyielding support in the animal and imaging facilities. You guys are the real MVPs and without your contributions, there is no doubt that this work would not have been possible. Mandy, $85 \%$ of this dissertation would NOT have happened had you not helped me in troubleshooting the Sweptfield for months on end, and I thank you tremendously for that.

To my incredible family, no amount of words could truly express the gratitude I have for the amount of love and support you all have shown me throughout my time in graduate school. I know the decision for us to move from the Texas panhandle to West Virginia was a tough one to handle, but you each supported and pushed me to finish strong and I am so grateful for that. Mom and Dad, I could not have asked for two better role models. You both instilled in me the foundation to believe in myself, to reach for my dreams and to never give up, and to above all keep God first. Your love, support, faith, and example have unquestionably shaped who I am today and I cannot thank you enough for that. This most certainly would not have been possible without your love and support. To Buddy and Shawn, thank you for accepting me into your incredible family and for loving me so fiercely. Without your immeasurable support and constant encouragement, the last three years would not have been as easy as they were. To my sweet Nana and Grandmommy, thank you both for the endless amounts of love you imparted on me anytime we talked. You both are so much of who I am, and I am so grateful to have been raised with such strong, female influences. I love you both more than I can say. I only 
wish Papa and Granddaddy were here to see this. To Lance, Lexie, Kody, Keri, Lyndsey, and Lucas, thank you all for your witty humor, the perfect group texts, and the countless prayers you guys have said on my behalf regarding any exam, qualifier, or presentation I endured. I am so fortunate to have been blessed with such incredible siblings and siblings-in-law.

To Pat, thank you for the (enormous amount of) emotional support you have provided to me for the last 10 years. You are the other pillar in the foundation of my being, and I have never ceased in thanking the Lord for placing you in my life. Thank you for the constant prayers and unwavering support you have shown me. I love you tremendously.

To my incredible, selfless, servant hearted, stud of a husband, Ryan, the past few years have not been without their stresses, but you have never ceased in providing a stress-free home full of laughter and joy. There were many times over the last year alone that I wanted to give in, but you never stopped encouraging me to achieve all that I could. I would not be pursing the career path that I am if I did not have the enormous amount of support from you that you've relentlessly demonstrated over the course of our marriage. Thank you for being my best friend, my favorite date, the one who makes me laugh like none other, and my strongest supporter. You are the peace that calms my crazy and my tangible grace. You make me a better person, and I feel so honored to be your wife. 
Finally, but most importantly, I would like to thank the Lord for the opportunity and blessing I was given at WVU, and for the irreplaceable relationships I have made along the way. I owe all that I am and have to God, and I pray to always reflect that in my life and career.

"And let us not grow weary of doing good, for in due season we will reap, if we do not give up." Galatians 6:9 


\section{TABLE OF CONTENTS}

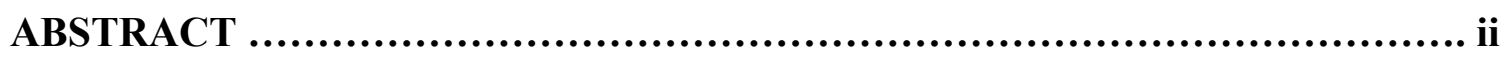

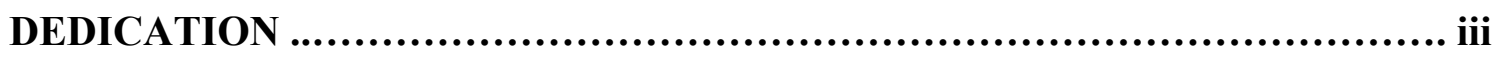

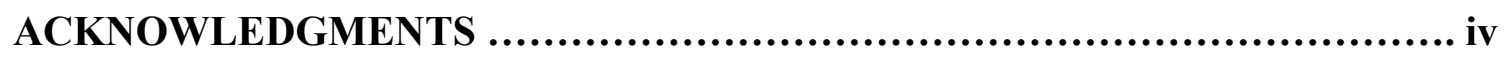

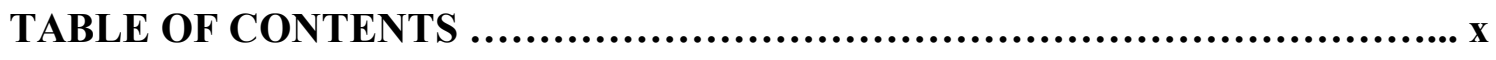

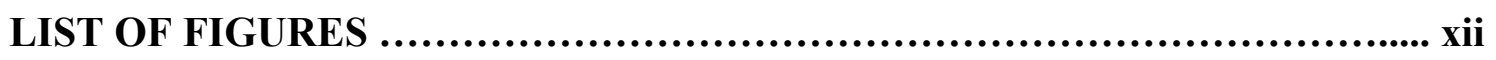

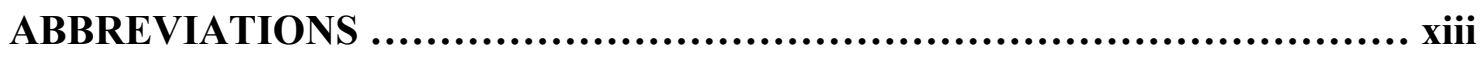

\section{CHAPTER}

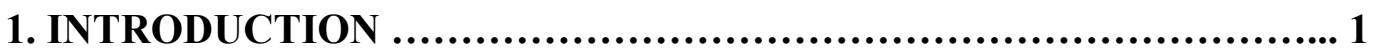

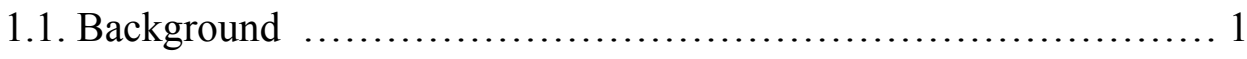

1.2. Chapter Summaries …....................................... 2

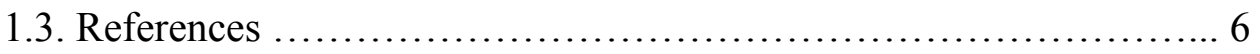

2. MODELING THE BLOOD-TUMOR BARRIER; A

REVIEW OF THE MOST COMMON IN VITRO

DEVICES .............................................................. 9

2.1. The Blood-Brain Barrier ........................................ 9

2.2. The Blood-Tumor Barrier …................................. 12

2.3. In Vitro Models ................................................... 12

2.4. Static Model: Transwells and Their Limitations..................... 16

2.5. Microfluidic Models ........................................... 17

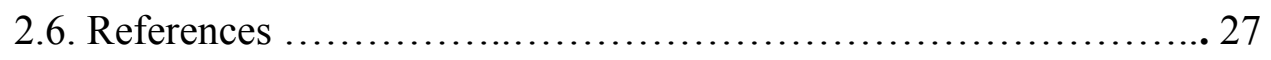

\section{PERMEABILITY ACROSS THE BLOOD-BRAIN}


BARRIER AND BLOOD-TUMOR BARRIER; A

NOVEL IN VITRO MODEL ON A CHIP .......................... 40

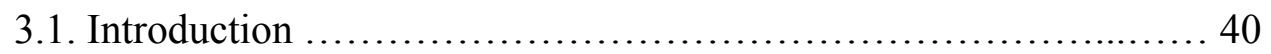

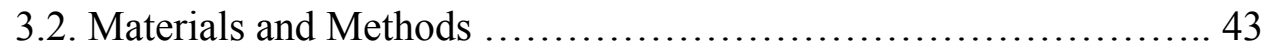

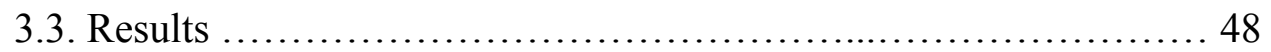

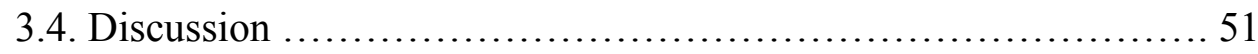

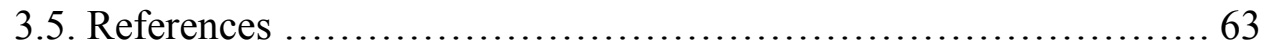

\section{TRASTUZUMAB EFFICACY IN AN IN VIVO}

AND IN VITRO MODEL OF BRAIN METASTASES

OF BREAST CANCER ............................................. 74

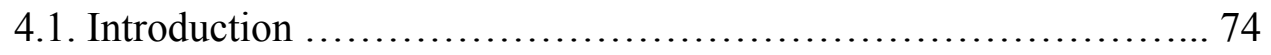

4.2. Materials and Methods ........................................ 77

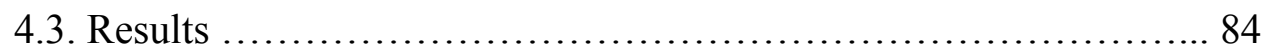

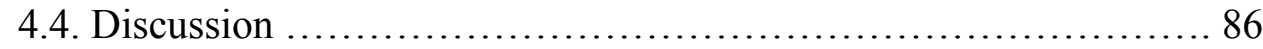

4.5. References ............................................ 94

5. CONCLUSIONS AND FUTURE DIRECTIONS .................... 103

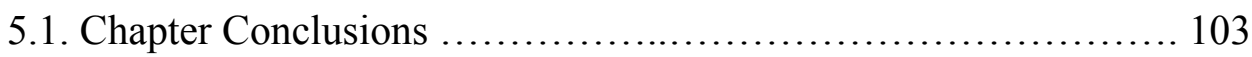

5.2. Future Directions ....................................... 105

5.3. References ............................................. 116

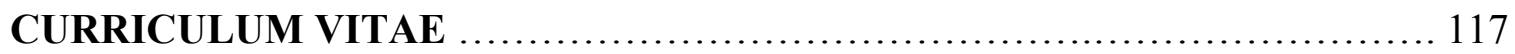

\section{LIST OF FIGURES}


3.1: Microfluidic chip schematic and methods

3.3: Diffusion rates of different sized MW tracers in the same model 58

3.4: Representative time-lapse images showing passive diffusion of Free TRD from the outer to the central compartment 59

3.5: Linear central compartment accumulation of different tracers in the BBB and BTB microfluidic chip models

3.6: Rhodamine- 123 permeability with and without inhibitors in BBB and BTB models

4.1: Mechanism of trastuzumab movement 90

4.2: The distribution of radiolabeled ${ }^{125} \mathrm{I}$-trastuzumab in various body organs, and in normal and tumor brain tissues

4.3: Heterogeneous and limited distribution of ${ }^{125}$ I-trastuzumab in preclinical brain metastases of breast cancer model 92

5.1: Microfluidic BTB device overall modifications 110

5.2: Re-engineering of the BTB microfluidic device to allow for real-time TEER measurements

5.3: Port volumes of the current microfluidic device

5.4: Proposed redesign for increased recovery port volumes of the microfluidic device

5.5: Tumor cell extravasation ................................................ 114

5.6: Isolation of different cells of varying metastatic potential 115 


\section{ABBREVIATIONS}

CNS - Central Nervous System

BTB - Blood-tumor barrier

BBB - Blood-Brain Barrier

TEER - Transendothelial Electrical Resistance

PDMS - Polydimethylsiloxane

bEnd3 - Brain endothelial cells

PC - Polycarbonate

ACM - astrocyte-conditioned medium

p-gp - p-glycoprotein

Rho123 - Rhodamine-123

HBMEC - human brain microvascular endothelial cells

Free TRD - Sulforhodamine 101 Acid Chloride

TRD 3 kDa - Texas Red 3000 MW Dextran

TRD 70 kDa - Texas Red 70,000 MW Dextran

HUVECs - Human Umbilical Vein Endothelial Cells

EBM-2 - Endothelial Basal Medium - 2

$\mathrm{k}_{\text {in }}-$ Unidirectional uptake transfer constants

t-Rho123 - fluorescent trastuzumab-Rho123

TRD $625 \mathrm{Da}$ - Texas red conjugated $625 \mathrm{MW}$ dextran

eGFP - enhanced green fluorescent protein

IP - intraperitoneal

PDXs - patient derived xenografts 


\section{CHAPTER 1}

\section{INTRODUCTION}

\subsection{Background}

One of the most critical factors concerning advanced stage breast cancer is the incidence of brain metastases. The incidence of brain metastases has risen significantly over the past 12 years (Carey, Ewend et al. 2004, Clayton, Danson et al. 2004, Lin, Bellon et al. 2004, Tham, Sexton et al. 2006, Smid, Wang et al. 2008, Olson, AbdelRasoul et al. 2013), probably due to successful treatements of the primary disease, leading to longer survival times and allowing for an increase in peripheral disease to occur. In advanced stage breast cancer, $10-16 \%$ of patients develop brain metastases (Palmieri, Smith et al. 2006) making breast cancer the second most common cause of metastatic brain tumors after lung cancer (10-25\%) (Steeg, Camphausen et al. 2011, Lin 2013, Yeh, Yu et al. 2015). Once a clinically detectable brain metastases is discovered and the patient becomes symptomatic, median survival is approximately 4 months (Colzani, Liljegren et al. 2011) with less than 2\% of women surviving two years postdiagnosis (Zimm, Wampler et al. 1981).

Despite the increased incidence, and the poor survival prognosis, treatment options are limited. Once diagnosed with a central nervous system (CNS) metastases, traditional treatment options have included radiation, surgery, and adjunctive systemic 
therapy. With regard to chemotherapy, inadequate drug delivery to tumors is because of poor penetration of drugs across the blood-tumor barrier (BTB) (Lockman, Mittapalli et al. 2010). This could be due to both poor permeability and an inadequate distribution to cross the BTB, or to the active efflux of the chemotherapeutics due to efflux transporters along the BTB and the individual tumor cells, actively removing the drugs from the brain parenchyma and back into the bloodstream once they have successfully crossed the BTB (Adkins, Mittapalli et al. 2013). Preclinical development of novel drugs to address these issues ultimately fail in clinical trials due to the unavailability of in vitro models that successfully predict or mimic the in vivo BTB.

The premise of this dissertation builds upon this significant body of literature demonstrating a failure of current chemotherapy regimens in the treatment of brain metastases of breast cancer due to poor distribution across the BTB. The specific aims of this dissertation were to: (1) Determine the permeability of three passive markers and one subject to efflux, in blood-brain barrier (BBB) and BTB models (2) Determine if trastuzumab crosses the $\mathrm{BBB}$ and $\mathrm{BTB}$ barrier through both in vivo and in vitro models (3) Evaluate if this microfluidic BBB and BTB models are relevant and compatible to current in vivo models.

\subsection{Chapter Summaries}

\subsubsection{Chapter 2}

There are multiple preclinical in vitro models available, however the limitations of each result in models that do not adequately mimic the in vivo BBB and BTB. In this 
chapter we thoroughly review the current static and microfluidic in vitro BBB and BTB devices, as well as each of their limitations in preclinical research.

\subsubsection{Chapter 3}

The lack of translatable in vitro BTB models creates challenges in the development of drugs to treat tumors of the CNS and our understanding of how the vascular changes at the BBB in the presence of a tumor. In this study, we characterize a novel microfluidic model of the BTB model (and BBB model as a reference) that incorporates flow that induces shear stress on endothelial cells. Cell lines utilized include human umbilical vein endothelial cells co-cultured with CTXTDR2 rat astrocytes (BBB) or Met-1 metastatic murine breast cancer cells (BTB). Cells communicated across microfluidic compartments via a porous interface. We characterized the device by comparing permeability of three passive permeability markers, and one marker subject to efflux. The permeability of Sulforhodamine 101 was significantly $(\mathrm{p}<0.05)$ higher in the BTB model $\left(13.1 \pm 1.3 \times 10^{-3}, \mathrm{n}=4\right)$ than the BBB model $\left(2.5 \pm 0.3 \times 10^{-3}, \mathrm{n}=6\right)$. Similar permeability increases were observed in the BTB model for molecules ranging from 600Da to 60kDa. The function of p-gp was intact in both models and consistent with recent published in vivo data. Specifically the rate of permeability of Rhodamine- 123 across the BBB model $(0.6 \pm$ $\left.0.1 \times 10^{-3}, \mathrm{n}=4\right)$, increased 14 -fold in the presence of verapamil $\left(14.7 \pm 7.5 \times 10^{-3}\right.$, $\mathrm{n}=3$ ) and eight fold with the addition of cyclosporine A ( $\left.8.8 \pm 1.8 \times 10^{-3}, \mathrm{n}=3\right)$. Similar values were noted in the BTB model. The dynamic microfluidic in vitro BTB 
model is a novel commercially available model that incorporates shear stress, and has permeability and efflux properties that are similar to in vivo data.

\subsubsection{Chapter 4}

Drug and antibody delivery to brain metastases has been highly debatable in the literature. The BTB, thought to be somewhat more permeable than the BBB, has shown to exhibit highly functioning efflux transporters and barrier functions, limiting delivery of these targeted therapies. The purpose of this study was to test the permeability of (1) I ${ }^{125}$ trastuzumab in an in vivo, and (2) fluorescent trastuzumab-Rho123 (t-Rho123) in a novel in vitro $\mathrm{BBB}$ and $\mathrm{BTB}$ brain metastases of breast cancer model. In vivo: Human MDAMB-231-HER2+ metastatic breast cancer cells were grown and maintained under static conditions. Cells were harvested at $80 \%$ confluency and prepped for intracardiac injection into 20 homozygous female $\mathrm{NuNu}$ mice. In vitro: Human umbilical vein endothelial cells were grown and maintained under shear stress conditions, co-cultured with CTX-TDR2 rat brain astrocytes (BBB) or Met-1 metastatic HER2+ murine breast cancer cells (BTB), grown and maintained under static conditions, across a porous interface in the outer and central compartments, respectively. Tissue distribution of ${ }^{125} \mathrm{I}$ trastuzumab revealed only $\sim 3 \%$ of injected dose reached normal brain, with $\sim 5 \%$ of injected dose reaching the brain tumor. No clear correlation was observed between size of metastases and the amount of ${ }^{125}$ I-trastuzumab localized in vivo. This heterogeneity was paralleled in vitro, where the distribution of t-Rho123 from the outer chamber to the central chamber of the microfluidic device was qualitatively and quantitatively analyzed

over time. The rate of t-Rho123 linear uptake in the BBB $\left(0.27 \pm 0.33 \times 10^{4}\right)$ and BTB 
$\left(1.29 \pm 0.93 \times 10^{4}\right)$ showed to be significantly greater than $0(\mathrm{p}<0.05)$. The BTB devices showed significant heterogenetic tendencies, as seen in in vivo. This study is one of the first studies to measure antibody movement across the blood-brain and bloodtumor barriers, and demonstrates that, though minute, trastuzumab does cross the bloodbrain and blood-tumor barriers.

\subsubsection{Chapter 5}

The conclusions of each chapter are discussed as well as the results of chapters 3 and 4 , in the form of modifications to the microfluidic device. These modifications were developed due to complications and failed experiments within the projects of chapter 3 and chapter 4. These modifications will result in improvements of different aspects of the device, leading to the future directions of this dissertation. 


\subsection{References}

Adkins, C. E., R. K. Mittapalli, V. K. Manda, M. I. Nounou, A. S. Mohammad, T. B. Terrell, K. A. Bohn, C. Yasemin, T. R. Grothe, J. A. Lockman and P. R. Lockman (2013). "P-glycoprotein mediated efflux limits substrate and drug uptake in a preclinical brain metastases of breast cancer model." Front Pharmacol 4: 136.

Carey, L. A., M. G. Ewend, R. Metzger, L. Sawyer, E. C. Dees, C. I. Sartor, D. T. Moore and M. L. Graham (2004). "Central nervous system metastases in women after multimodality therapy for high risk breast cancer." Breast Cancer Res Treat 88(3): 273280.

Clayton, A. J., S. Danson, S. Jolly, W. D. Ryder, P. A. Burt, A. L. Stewart, P. M. Wilkinson, R. S. Welch, B. Magee, G. Wilson, A. Howell and A. M. Wardley (2004). "Incidence of cerebral metastases in patients treated with trastuzumab for metastatic breast cancer." Br J Cancer 91(4): 639-643.

Colzani, E., A. Liljegren, A. L. Johansson, J. Adolfsson, H. Hellborg, P. F. Hall and K. Czene (2011). "Prognosis of patients with breast cancer: causes of death and effects of time since diagnosis, age, and tumor characteristics." J Clin Oncol 29(30): 4014-4021.

Lin, N. U. (2013). "Breast cancer brain metastases: new directions in systemic therapy." ecancermedicalscience 7: 307. 
Lin, N. U., J. R. Bellon and E. P. Winer (2004). "CNS metastases in breast cancer." J Clin Oncol 22(17): 3608-3617.

Lockman, P. R., R. K. Mittapalli, K. S. Taskar, V. Rudraraju, B. Gril, K. A. Bohn, C. E. Adkins, A. Roberts, H. R. Thorsheim, J. A. Gaasch, S. Huang, D. Palmieri, P. S. Steeg and Q. R. Smith (2010). "Heterogeneous blood-tumor barrier permeability determines drug efficacy in experimental brain metastases of breast cancer." Clin Cancer Res 16(23): 5664-5678.

Olson, E. M., M. Abdel-Rasoul, J. Maly, C. S. Wu, N. U. Lin and C. L. Shapiro (2013). "Incidence and risk of central nervous system metastases as site of first recurrence in patients with HER2-positive breast cancer treated with adjuvant trastuzumab." Ann Oncol 24(6): 1526-1533.

Palmieri, D., Q. R. Smith, P. R. Lockman, J. Bronder, B. Gril, A. F. Chambers, R. J. Weil and P. S. Steeg (2006). "Brain metastases of breast cancer." Breast Dis 26: 139-147.

Smid, M., Y. Wang, Y. Zhang, A. M. Sieuwerts, J. Yu, J. G. Klijn, J. A. Foekens and J. W. Martens (2008). "Subtypes of breast cancer show preferential site of relapse." Cancer Res 68(9): 3108-3114.

Steeg, P. S., K. A. Camphausen and Q. R. Smith (2011). "Brain metastases as preventive and therapeutic targets." Nat Rev Cancer 11(5): 352-363. 
Tham, Y. L., K. Sexton, R. Kramer, S. Hilsenbeck and R. Elledge (2006). "Primary breast cancer phenotypes associated with propensity for central nervous system metastases." Cancer 107(4): 696-704.

Yeh, R. H., J. C. Yu, C. H. Chu, C. L. Ho, H. W. Kao, G. S. Liao, H. W. Chen, W. Y. Kao, C. P. Yu, T. Y. Chao and M. S. Dai (2015). "Distinct MR Imaging Features of Triple-Negative Breast Cancer with Brain Metastasis." J Neuroimaging 25(3): 474-481.

Zimm, S., G. L. Wampler, D. Stablein, T. Hazra and H. F. Young (1981). "Intracerebral metastases in solid-tumor patients: natural history and results of treatment." Cancer 48(2): 384-394. 
CHAPTER 2

MODELING THE BLOOD-TUMOR BARRIER; A REVIEW OF THE MOST COMMON IN VITRO DEVICES

\subsection{The Blood-Brain Barrier}

\subsubsection{Cellular Function}

The blood-brain barrier (BBB) is a highly controlled and strictly regulated complex network of brain microvessels. This barrier is the primary protective interface for the brain that functionally restricts ions, molecules, toxins and drug movement from blood to the brain parenchyma (Almutairi, Gong et al. 2016). The protective nature of the $\mathrm{BBB}$ is due in part to the multicellular system (neurovascular unit) that forms it, consisting of microvascular brain endothelial cells surrounded by astrocytic foot processes, pericytes, neurons, and microglia (Wolff, Antfolk et al. 2015). The first, and primary cellular unit of the neurovascular unit are the microvascular brain endothelial cells. These specific endothelial cells are noted for their absence of fenestrae and the presence of a continuous basement membrane (shared with pericytes) (de Boer and Gaillard 2006). These two components are key elements to the highly restrictive nature across microvascular endothelial cells when compared to normal endothelial cells. Brain microvascular endothelial cells also express a higher than normal amount of tight junctions as well as limited pinocytic vesicular transport (Abbott, Patabendige et al. 2010, Wolff, Antfolk et al. 2015). 
The next cellular structure of the neurovascular unit family are the astrocytes. Found only in the brain, these cells provide much needed biochemical support to the BBB through contact to the microvascular brain endothelial cells with their multiple foot processes (Taber and Hurley 2008). This biochemical support has been previously studied, and has well established the importance of astrocytes to the integrity of the BBB (Abbott 2002) and paracellular movement (Zheng, Aschner et al. 2003), as well as the secretion of various factors required for successful BBB function (Janzer and Raff 1987, Siddharthan, Kim et al. 2007, Colgan, Collins et al. 2008).

Pericytes are the third cellular structure, and they cover approximately $22 \%-33 \%$, or one fifth to one third, of the basolateral portion of a capillary (Kim, Tran et al. 2006). Pericytes have multiple functions; they have been shown to induce the polarity of astrocytes leading to a tightening of the BBB (Allt and Lawrenson 2001), affect the integrity of the BBB through direct contact with the endothelial cells (Hayashi, Nakao et al. 2004), and may play a role in angiogenesis (Daneman, Zhou et al. 2010). Pericytes also inhibit the expression of molecules known to increase vascular permeability (Daneman, Zhou et al. 2010), while a deficiency of pericytes has been linked to an increase in permeability of the BBB (Armulik, Genove et al. 2010).

The last two cells related to the neurovascular unit composing the BBB are neurons and microglia. Neurons, the main functional cells of the brain, communicate through different chemical and electrical signals. These signals rely on the movement of small ions, which aids in the overall maintenance of stable membrane potentials (Abbott 
2013). The presence of neurons has also been shown to increase the integrity of the BBB (Minami 2011), which helps maintain the homeostasis of the brain, and protect the brain from the influx and efflux of ions. Microglia, the final member of the neurovascular unit, are found in the perivascular space and are the immune cells of the central nervous system (CNS), meaning they simply clear away debris and apoptotic cells from the brain (Sumi, Nishioku et al. 2010).

\subsubsection{Restrictions and Permeability}

Within the neurovascular unit, tight junctions at the level of the endothelia play a major role in the protective nature of the BBB. Tight junctions are a hallmark of the BBB, and are composed of claudins, occludins, and junctional adhesion molecules. These junctions seal the microvascular endothelial cells together, creating a physical barrier that aids in the regulation of drugs, oxygen, nutrients, ions and pathogens from systemic circulation to the brain (Petty and Lo 2002). Tight junctions also restrict paracellular transport, or the movement through the intracellular space between cells. Paracellular transport is a passive pathway and relies on concentration gradients and permeability, causing it to be a slow method of transport (Pardridge 1999). Due to tight junctions tightly restricting the paracellular movement between endothelial cells, an alternative mechanism to circumvent the BBB is through transcellular transport. Transcellular pathways, or the movement through a cell, are energy dependent and substrate specific (Mager, Meyer et al. 2016). For example, lipophilic molecules can cross the BBB through transendothelial receptor-mediated transport (Schinkel 1999, Vorbrodt and Dobrogowska 2003, Roberts, Black et al. 2008, Abbott, Patabendige et al. 2010). 


\subsection{The Blood-Tumor Barrier}

Once a metastatic tumor cell extravasates from the primary tumor location, it enters the bloodstream and travels to a secondary location, where it embeds and begins to establish its own blood supply (Carmeliet and Jain 2000, Talmadge and Fidler 2010, Eichler, Chung et al. 2011). This process causes a change in the barrier, from the bloodbrain barrier to the blood-tumor barrier (BTB). A tumor's blood supply may develop through a couple of mechanisms: vasculogenesis, angiogenesis, and co-option being the main forms. During development, new blood vessels are formed in a process called vasculogenesis. Tumor cells have been observed mimicking endothelial cells and forming vascular vessels themselves through the use of cancer stem cells or tumor initiating cells (Krishna Priya, Nagare et al. 2016). Angiogenesis is the secretion of vascular endothelial growth factor by the tumor cell to form sprouts from existing blood vessels (Carmeliet and Jain 2000, Folkman 2007, Carmeliet and Jain 2011). Tumor cells can also grow along an existing blood vessel instead of sprouting new blood vessels in a mechanism known as co-option (Frentzas, Simoneau et al. 2016). After a tumor establishes a blood supply, the only thing left is to grow. Tumor permeability, or the leakiness of a tumor, is affected by how many fenestra are present in the BTB, and the spatial distribution of, or distance between, the tumor vasculature.

\subsection{In Vitro Models}

\subsubsection{Basic Premise}

The best way to study molecular transport across the BBB is in vivo, or studies in the animal's natural environment, however only $\sim 50 \%$ of these results are translational to 
human (Perel, Roberts et al. 2007). Since in vivo studies are difficult and expensive, in vitro models are utilized. In vitro BBB models should, ideally, mimic the structural and functional properties of the in vivo $\mathrm{BBB}$ and meet the following four requirements: 1) Tight junction expression should result in a very restricted barrier. 2) Correct placement (luminal vs. abluminal) of influx and efflux transporters, as well as the functionality of each transporter compared to the in vivo BBB (Roberts, Black et al. 2008). 3) Permeability across the BBB and BTB should be comparable to in vivo (Adkins, Mittapalli et al. 2013). 4) Replication of shear stress and vascular flow (Ballermann, Dardik et al. 1998, Tarbell 2010, Cucullo, Hossain et al. 2011).

Shear stress and vascular flow dramatically alters the morphology of endothelial cells when compared to static cells (Cucullo, Hossain et al. 2011, Prabhakarpandian, Shen et al. 2013, Deosarkar, Prabhakarpandian et al. 2015) (SynVivo Chapter 3). Without the added shear stress of vascular flow, endothelial cell morphology is described as flat and small, with an increased presence of endocytic vesicles, microfilaments and clatherin-coated pits (Ballermann and Ott 1995). However, through the addition of vascular flow, shear stress alters the endothelial cellular morphology through the elongation and increase in size of the endothelia, as well as a decrease in the presence of endocytic vesicles, microfilaments, and clatherin-coated pits. There is documented evidence also showing a correlated increase in the strength of tight junctions between endothelial cells with the addition of shear stress (Collins, Cummins et al. 2006, Colgan, Ferguson et al. 2007, Siddharthan, Kim et al. 2007). 
Every in vitro model includes the same basic parameters: barrier cells, extracellular matrix, and a brain microenvironment (Stanimirovic, Bani-Yaghoub et al. 2015). The barrier cells are grown on semipermeable membrane dividing the basolateral and apical compartments, which are located on one side (apical) of the extracellular matrix. The extracellular matrix is located on a semipermeable membrane, which separates the brain microenvironment (basolateral) and the barrier cells. When studying permeability, drugs are placed in the apical compartment and the movement is measured in the basolateral compartment, and studied over time. These models have previously been studied in a 6-96 well format, and can be miniaturized through the use of microfluidics to increase throughput and mimic shear stress as seen in vivo.

\subsubsection{Cell Culture Options}

In any in vitro system, there is the option of monoculture, co-culture, or triple culture of the cells (Wolff, Antfolk et al. 2015). One of the most widely and easily used in vitro systems is a monoculture system. In this system, the only cells used are endothelial cells, grown on the apical side of the model, occasionally with the addition of astrocyte-conditioned media, which has been shown to increase barrier function (Siddharthan, Kim et al. 2007). However, despite having only one cell type, the absence of astrocytes makes this model undesirable due to the vast amount of data showing the importance of astrocytes to the integrity of the BBB (Abbott 2002, Abbott, Patabendige et al. 2010, Abbott 2013). The next step in the in vitro model system is the co-culture system: contact co-culture or noncontact co-culture. Depending on the type of model, the transendothelial electrical resistance (TEER) will vary. TEER is a quantitative technique 
used to measure the tightness of the seal between endothelial cells (created by tight junctions) and is a good indicator of the functionality of the BBB. The higher the TEER value, the stronger the integrity of the barrier is thought to be. In a contact co-culture system, endothelial cells are grown as in the monoculture model, with astrocytes/pericytes/neurons grown on the bottom of the porous membrane (if in a transwell) or in the basolateral chamber, where they are able to have direct contact with the endothelial cells. Through direct contact with the secondary cells, the TEER values for endothelial cells have been shown to increase by upwards of nine times higher, in comparison, to the monoculture model (Gaillard, Voorwinden et al. 2001, Nakagawa, Deli et al. 2009).

In contrast, a noncontact co-culture system is where the astrocytes/pericytes/neurons are grown on the bottom of the 6-96 well plate, not in direct contact with the endothelial cells. Morphological, chemical, and biological changes can be observed due to the chemical gradient, with TEER values reportedly increased by a factor of 2 in comparison to the monoculture model (Nakagawa, Deli et al. 2009). The last, most complicated, model is the triple culture. There are a couple of options with this model: 1) Endothelial cells are in direct contact with astrocytes and indirect contact with neurons, showing a $35.9 \%$ increase in TEER when compared to monoculture (Xue, Liu et al. 2013). 2) Endothelial cells are in direct contact with pericytes and indirect contact with astrocytes, where an eight-fold increase in TEER is observed in comparison to the monoculture model (Nakagawa, Deli et al. 2007). 


\subsection{Static Model: Transwells and Their Limitations}

When choosing an in vitro model, the desire is to find a model that cultures pure cell types resulting in high TEER values with low permeability. There are a couple options when choosing an in vitro $\mathrm{BBB} / \mathrm{BTB}$ model: static or microfluidic models. Transwells are the most widely used and commercially available static in vitro models to study diffusional movement across endothelia are transwell systems. Briefly, it consists of an upper chamber with endothelia grown on top of a porous membrane, which resides above a lower chamber containing astrocytes/neurons for a blood-brain barrier (BBB) model and cancer cells for a BTB model (Bicker, Alves et al. 2014, Czupalla, Liebner et al. 2014, Srinivasan, Kolli et al. 2015, Helms, Abbott et al. 2016). Permeation of molecules and or cells is evaluated by calculating accumulation/distribution between compartments.

The transwell model has limitations that result in calculation errors in drug movement, as well as increased apparent permeability rates due to the endothelia (upper chamber) having gaps between cells near the insert edge (Noseda, Chang et al. 2004, Santaguida, Janigro et al. 2006), and because of the lack of flow associated sheer stress which decreases tight junction formation (Prabhakarpandian, Shen et al. 2013, Czupalla, Liebner et al. 2014, Deosarkar, Prabhakarpandian et al. 2015, Helms, Abbott et al. 2016). Another limitation is the ten-fold difference in drug diffusion calculations when compared to "proportional" in vivo measurements due to the presence of an unstirred water layer above the endothelial surface. This unstirred water layer results in increased permeability for hydrophilic drugs and decreased permeability for lipid soluble drugs 
(Barry and Diamond 1984, Noseda, Chang et al. 2004, Prabhakarpandian, Shen et al. 2013, Czupalla, Liebner et al. 2014, Deosarkar, Prabhakarpandian et al. 2015).

\subsection{Microfluidic Models}

Microfluidic devices are still early in the BBB field and aren't as widely used as transwells due to their price, lack of commercial availability, and issues for researchers to master (Stanness, Guatteo et al. 1996, Lippmann, Azarin et al. 2012). Despite not being as well used, the basic premise for most microfluidic devices is still the same. As with transwells, endothelial cells are co-cultured in a luminal (apical) compartment with astrocytes, or the secondary cells of choice, are seeded on the basolateral side of the lumen. After all cells have been established and allowed to grow in static conditions, vascular flow is exerted in the apical chamber for a set amount of time, and permeability studies are allowed to commence through the flow of tracer through the apical chamber with samples taken from the basolateral chamber.

In recent years static co-culture has begun to fall by the wayside with the advent of more physiologically relevant models. There has been a great deal of work in the realms of microfluidics, or lab-on-a-chip technologies, in order to address some of the known shortcomings of other in vitro models. The push to establish a reliable and replicable microfluidic BBB has quickly produced a wide variety of models, most of which incorporate consistent flow in order to promote the expression of tight junctions in cultured endothelial cells and more closely mimic the in vivo $\mathrm{BBB}$, though these models are not without their own difficulties and shortcomings (van der Helm, van der Meer et 
al. 2016). To characterize these models, investigators employ similar quantifying methods as those used to characterize transwells and other static cultures. These methods include: TEER, immunofluorescent staining of tight junctions, cell viability assays, and measurement of the permeability of various compounds, which may then be compared to in vivo values and other static in vitro models (Lockman, Mittapalli et al. 2010, Adkins, Mittapalli et al. 2013, Thomsen, Burkhart et al. 2015). For this review, we have selected a number of models representing the wide diversity of approaches to creating a microfluidic BBB.

\subsubsection{Stacked Compartmental Designs}

One of the first viable models of microfluidic tissue culture developed specifically to model the BBB was developed by Booth and Kim in 2012 (Booth and Kim 2012). Their model utilized stacked polydimethylsiloxane (PDMS) culture chambers separated by a perforated membrane. These separate chambers allowed for separate culture of astrocytes (C8D1A) and brain endothelial cells (bEnd3), as well as their biochemical communication through the membrane, which has also been previously observed with transwell models (Booth and Kim 2012). Electrodes were incorporated to allow for TEER measurements. Nearly all endothelial cells seeded in the device remained viable and expressed tight junctions after 3 days under continuous flow of $2.6 \mathrm{~mL} \mathrm{~min}{ }^{-1}$ (Booth and Kim 2012). Their static control, traditional transwells, exhibited TEER values around $25 \Omega \mathrm{cm}^{2}$, and a significant increase in TEER when dynamic flow was incorporated in their chip model, over $250 \Omega \mathrm{cm}^{2}$ (Booth and Kim 2012). They also showed a significant improvement in TEER values from a bEnd3 monolayer in their chip device, nearly $175 \Omega$ 
$\mathrm{cm}^{2}$, to devices in co-culture with steady-state TEER values at more than $250 \Omega \mathrm{cm}^{2}$ (Booth and Kim 2012).

Many other models utilize PDMS superstructure because it is optically transparent, inexpensive, can be adapted to virtually any 3D-printed silicon cast, and is relatively expedient in production, as a 10:1 mixture of base to curing agent can cure in approximately 1h (Yeon, Na et al. 2012, Achyuta, Conway et al. 2013, Griep, Wolbers et al. 2013, Prabhakarpandian, Shen et al. 2013, Brown, Pensabene et al. 2015, Cho, Seo et al. 2015, Deosarkar, Prabhakarpandian et al. 2015, Sellgren, Hawkins et al. 2015, Wang, Khafagy el et al. 2016). In 2013, Achyuta et al. also developed a vertically stacked PDMS compartment device. However, their approach required the separate culture of RBE4 endothelial and primary rat cortical cells in their respective chambers before stacking the components brought them into a co-culture environment, in which the flow over the endothelial cells was approximately $17 \mu \mathrm{L} \mathrm{min} \operatorname{mon}^{-1}$ (Achyuta, Conway et al. 2013). The TEER of the barriers in these devices could not be measured, as no electrodes were incorporated into the device, but the in vitro BBB displayed appropriate physiological responses to TNF $\alpha$ stimulation, namely increased permeability to a $3 \mathrm{kDa}$ Alexafluor $^{\mathrm{TM}}$ conjugated dextran (Achyuta, Conway et al. 2013). Endothelial cells were also confirmed to express tight-junction protein ZO-1, which was elevated in the presence of astrocyteconditioned medium (ACM) (Achyuta, Conway et al. 2013).

One of the most recent stacked PDMS devices was developed by Sellgren et al. in 2015. They also cultured cells in stacked PDMS chambers separated by $0.4 \mu \mathrm{m}$ porous 
membranes of two different materials: nanopourous Teflon and polycarbonate (PC). Rather than seeding their C8D1A astrocytes on one side of the membrane directly, they suspended the cells in a collagen matrix and injected them into the device before seeding the bEnd 3 cells on the opposite side of the membrane (Sellgren, Hawkins et al. 2015). The endothelial cell chamber was $150 \mu \mathrm{m}$ in diameter, the size of a vessel much larger than human brain capillaries, which average approximately $10 \mu \mathrm{m}$ in diameter (Wong, Ye et al. 2013). However, they still achieved near physiological shear stress at $5 \mathrm{dyn} \mathrm{cm}^{-2}$ with a flow rate of $120 \mu 1 \mathrm{~min}^{-1}$. Their model displayed distinct tight junction expression through the staining of claudins, and also a significantly tighter barrier than those observed in static transwell cultures, and the two membrane materials were comparable (Sellgren, Hawkins et al. 2015).

Other stacked devices have been produced, and though they fail to recapitulate the cylindrical shape of capillaries and the brain tissue surrounding them, some have made very promising strides in the improvement of in vitro BBB models. Wang et al. fabricated a device using a 3D printer to deposit Objet VeroClear photopolymer to create a set of stacked compartments for cell culture and media storage (Wang, Khafagy el et al. 2016). Their model utilized a rocking table rather than a perfusion pump to move media across the surface of BMECs differentiated from human pluripotent stem cells, which were maintained in co-culture with primary rat astrocytes (Wang, Khafagy el et al. 2016). Without the magnitude of shear stress induced in other models to promote formation of tight junctions, their chip devices still maintained TEER values of $3000 \Omega \mathrm{cm}^{2}$ (Wang, 
Khafagy el et al. 2016), some of the closest to in vivo values seen in an in vitro model, which they credit to . This may be due to the origin of their cells, which is unique.

\subsubsection{Horizontal Layouts}

Another set of PDMS models have been designed with a horizontal layout. A number of microfluidic models have been developed using devices produced by SynVivo Corporation. Prabhakarpandian et al. created their SymBBB using a PDMS superstructure mounted to a glass microscope slide (Prabhakarpandian, Shen et al. 2013). It was seeded with rat brain endothelial cells (RBE4) and perfused with ACM at a rate of $0.1 \mu 1 \mathrm{~min}^{-1}$. Bifurcated apical chambers were separated from a central basolateral chamber by a $100 \mu \mathrm{m}$ section of PDMS perforated with $3 \mu \mathrm{m}$ gaps to allow for passive diffusion (Prabhakarpandian, Shen et al. 2013). Though their chip did not include electrodes for TEER evaluation, they could be imaged to show real-time diffusion and permeability of fluorescent tracer (Prabhakarpandian, Shen et al. 2013). They assessed transwell permeability of 3-5kDa FITC-dextran over time and compared it to their device, showing a significant decrease in permeability in the microfluidic model both with and without ACM (Prabhakarpandian, Shen et al. 2013). It was also observed that cells cultured in their device with ACM exhibited normal P-glycoprotein (p-gp) efflux of Rhodamine-123 (Rho123), and an increased permeability of Rho123 through the RBE4 endothelium in the presence of the p-gp inhibitor, verapamil (Prabhakarpandian, Shen et al. 2013). 
An additional set of experiments by Deosarkar et al. demonstrated the development of a neonatal rat BBB in another variation of a SynVivo microfluidic chip. The device contained two separate apical chambers and a circular central basolateral chamber, and these chambers were separated by a section of PDMS with the same $3 \mu \mathrm{m}$ gaps as the aforementioned device (Deosarkar, Prabhakarpandian et al. 2015). The devices were seeded with primary neonatal rat astrocytes and primary neonatal Rat brain endothelial cells which, after attachment, were maintained under a constant flow of 0.01 $\mu 1 \min ^{-1}$ (Deosarkar, Prabhakarpandian et al. 2015). To assess permeability, 40kDa Texas Red dextran was perfused into the device at a rate of $0.2 \mu 1 \mathrm{~min}^{-1}$ for 90 minutes. They performed these experiments as well as ICC on cells in a variety of environments (with primary astrocytes, with $\mathrm{ACM}$, and with primary neonatal rat brain endothelial cells alone) and found a significant decrease in permeability and an increase in the expression of tight junction protein ZO-1, which was dependent on the presence of astrocytes or ACM (Deosarkar, Prabhakarpandian et al. 2015). Barrier tightness was also assessed through electrical resistance and, again, increased with the presence of astrocytes and ACM (Deosarkar, Prabhakarpandian et al. 2015). However, these values cannot, as of now, be compared to TEER values from transwells or other models, due to the novel methodology and equipment used to acquire the measurements (Deosarkar, Prabhakarpandian et al. 2015).

\subsubsection{Hollow Fibrous Cellular Supports}

Another model, produced by Herland et al. in 2016, utilizes a combination of PDMS super structure and a hollow tube of collagen fibers for cell support. Within what 
amounts to a simple PDMS box, they used a collagen matrix with suspended human astrocytes to form a cylindrical vessel (Herland, van der Meer et al. 2016). They then seeded human pericytes in two stages, flipping the device over in each stage to form a full cylindrical monolayer of the cells, and followed with the seeding of human brain microvascular endothelial cells (HBMEC) (Herland, van der Meer et al. 2016). In this way, they created a softer, more physiologically relevant structure than other PDMS models while also incorporating all three structural components of brain microvessels (Herland, van der Meer et al. 2016). At a flow of $120 \mu \mathrm{L} \mathrm{min}^{-1}$, they achieved a shear stress of 1 dyne $\mathrm{cm}^{-2}$, which is low in comparison to physiological conditions.

They measured barrier tightness using $3 \mathrm{kDa}-A$ lexa488 Dextran diffusion, as TEER electrodes could not be reliably incorporated into their collagen gels (Herland, van der Meer et al. 2016). They also assessed the inflammatory response of the in vitro vessels to TNF $\alpha$ and stained for tight junction proteins (Herland, van der Meer et al. 2016). They then compared their dynamic model to transwells seeded with the same cells. Though their study focused on the particular effects of the presence of astrocytes or pericytes, rather than the creation of a physiologically relevant model with a sufficiently tight barrier, they did observe distinct expression of tight junctions (Herland, van der Meer et al. 2016). They also found that their model responded differently to inflammation than transwells, with the synthetic vasculature exhibiting a lower fold-increase in inflammatory cytokines released after stimulation with TNF $\alpha$ (Herland, van der Meer et al. 2016). 
Other models have built on a variety of materials for cell support. Cucullo et al. built upon previous capillary models (Cucullo, McAllister et al. 2002) and also used two sets of hollow polypropylene fibers housed in sealed chambers and connected by gas permeable silicon tubing (Cucullo, Hossain et al. 2013). The abluminal surface of each hollow fiber was coated with fibronectin to promote endothelial cell adhesion, and the outer surface was coated with poly-D-lysine to allow for the attachment of astrocytes (Cucullo, Hossain et al. 2013). In one chamber, which contained $n=3$ hollow fibers, HBMECs were seeded on the abluminal surface of each hollow fiber, and human astrocytes were seeded on the external surface (Cucullo, Hossain et al. 2013). In the other chamber, hollow fibers were seeded with HBMECs and human brain vascular smooth muscle cells rather than astrocytes (Cucullo, Hossain et al. 2013). These two chambers were sealed and connected with silicon tubing to a reservoir of media and a pulsatile pump, which delivered a flow of media which was gradually increased from a low shear stress of 1 dyne $\mathrm{cm}^{-2}$ to what was comparable to a physiologically relevant blood pressure (80-300mmHg)(Cucullo, Hossain et al. 2013).

This complex model of brain vasculature was then characterized by a variety of experiments: TEER, molecular permeability assays, response to a hyperosmolar agent, and the determination of metabolic activity (Cucullo, Hossain et al. 2013). TEER values achieved by this system approached $800 \Omega \mathrm{cm}^{2}$ in the capillary component, whereas the venule chamber did not achieve TEER above $250 \Omega \mathrm{cm}^{2}$ (Cucullo, Hossain et al. 2013). They measured permeability of Diazepam, phenytoin, and sucrose, showing a greater permeability of each of these molecules in the venule segment in comparison to the 
capillary compartment, an observation consistent with in vivo physiology (Cucullo, Hossain et al. 2013). When exposed to a hyperosmolar agent, in this case 1.6M mannitol, which is sometimes used to increase the permeability of chemotherapeutics, the agent induced same opening of the in vitro vascular model as has been observed in vivo (Cucullo, Hossain et al. 2013). Finally, their bioenergetic assessment revealed that the capillary segment favored aerobic respiration while the venule segment, which was exposed to lower shear stress, favored anaerobic respiration (Cucullo, Hossain et al. 2013). However, they note that further experiments should be completed to confirm these results and possible reasons for them.

\subsubsection{Cancer Monoculture and Drug Screening Design}

Other microfluidic devices aimed at brain cancer do not incorporate endothelial cells with BBB-like properties as a barrier. Rather they use only cancer cells to grow tumors under fluid flow to assess the effectiveness of various therapeutics. For example, in 2016, Fan et al. developed a microfluidic platform for high-throughput screening of the effectiveness of drugs on a glioblastoma cell line (Fan, Nguyen et al. 2016). They used poly(ethelene) glycol diacrylate superstructure, to more closely imitate an ECM, and grew U87 glioblastoma multiforme cells in sphereoid masses in each microwell within the chip (Fan, Nguyen et al. 2016). They then characterized diffusion of dyes to assess fluid dynamics, and they treated cells with the conventional chemotherpeutics irinotecan and pitavastatin individually and in combination. They observed predicted cell death, and they could determine drug concentrations at each time point. Their matrix, however, did 
absorb some of the drugs, and therefore released them back into the media over time, adding another unique variable to their device (Fan, Nguyen et al. 2016).

This type of model has the potential to expedite the introduction of personalized medicine and may be a high-throughput alternative for the screening of novel compounds, which may be highly effective at treating particular cancers. However, without the incorporation of BBB-like membranes to assess drug permeability and the ultimate concentrations which may be available after crossing the BBB, these assays are largely irrelevant for assessments of novel therapeutics metastatic brain cancers and for patients who have been diagnosed with them. Therefore, models that incorporate not only a cancer cell line, but also an endothelium with BBB-like properties, should be pursued.

In addition, though many of these models conceptually improve upon transwell culture by bypassing the issues of unstirred water layers and unreliable concentration gradients, and they incorporate other means of BBB recapitulation such as fluid flow, many of them do not significantly improve barrier tightness in comparison to some transwell models, which have recently reported maximum TEER values in excess of $1000 \Omega \mathrm{cm}^{2}$ (Patabendige, Skinner et al. 2013). Further, many are so complex, they require extended amounts of time to produce, seed, and reach the steady states necessary to perform experiments. 


\subsection{References}

Abbott, N. J. (2002). "Astrocyte-endothelial interactions and blood-brain barrier permeability." J Anat 200(6): 629-638.

Abbott, N. J. (2013). "Blood-brain barrier structure and function and the challenges for CNS drug delivery." J Inherit Metab Dis 36(3): 437-449.

Abbott, N. J., A. A. Patabendige, D. E. Dolman, S. R. Yusof and D. J. Begley (2010). "Structure and function of the blood-brain barrier." Neurobiol Dis 37(1): 13-25.

Achyuta, A. K., A. J. Conway, R. B. Crouse, E. C. Bannister, R. N. Lee, C. P. Katnik, A. A. Behensky, J. Cuevas and S. S. Sundaram (2013). "A modular approach to create a neurovascular unit-on-a-chip." Lab Chip 13(4): 542-553.

Adkins, C. E., R. K. Mittapalli, V. K. Manda, M. I. Nounou, A. S. Mohammad, T. B. Terrell, K. A. Bohn, C. Yasemin, T. R. Grothe, J. A. Lockman and P. R. Lockman (2013). "P-glycoprotein mediated efflux limits substrate and drug uptake in a preclinical brain metastases of breast cancer model." Front Pharmacol 4: 136.

Allt, G. and J. G. Lawrenson (2001). "Pericytes: cell biology and pathology." Cells Tissues Organs 169(1): 1-11. 
Almutairi, M. M., C. Gong, Y. G. Xu, Y. Chang and H. Shi (2016). "Factors controlling permeability of the blood-brain barrier." Cell Mol Life Sci 73(1): 57-77.

Armulik, A., G. Genove, M. Mae, M. H. Nisancioglu, E. Wallgard, C. Niaudet, L. He, J. Norlin, P. Lindblom, K. Strittmatter, B. R. Johansson and C. Betsholtz (2010). "Pericytes regulate the blood-brain barrier." Nature 468(7323): 557-561.

Ballermann, B. J., A. Dardik, E. Eng and A. Liu (1998). "Shear stress and the endothelium." Kidney Int Suppl 67: S100-108.

Ballermann, B. J. and M. J. Ott (1995). "Adhesion and differentiation of endothelial cells by exposure to chronic shear stress: a vascular graft model." Blood Purif 13(34): $125-134$.

Barry, P. H. and J. M. Diamond (1984). "Effects of unstirred layers on membrane phenomena." Physiol Rev 64(3): 763-872.

Bicker, J., G. Alves, A. Fortuna and A. Falcao (2014). "Blood-brain barrier models and their relevance for a successful development of CNS drug delivery systems: a review." Eur J Pharm Biopharm 87(3): 409-432.

Booth, R. and H. Kim (2012). "Characterization of a microfluidic in vitro model of the blood-brain barrier (muBBB)." Lab Chip 12(10): 1784-1792. 
Brown, J. A., V. Pensabene, D. A. Markov, V. Allwardt, M. D. Neely, M. Shi, C. M. Britt, O. S. Hoilett, Q. Yang, B. M. Brewer, P. C. Samson, L. J. McCawley, J. M. May, D. J. Webb, D. Li, A. B. Bowman, R. S. Reiserer and J. P. Wikswo (2015). "Recreating blood-brain barrier physiology and structure on chip: A novel neurovascular microfluidic bioreactor." Biomicrofluidics 9(5): 054124.

Carmeliet, P. and R. K. Jain (2000). "Angiogenesis in cancer and other diseases." Nature 407(6801): 249-257.

Carmeliet, P. and R. K. Jain (2011). "Molecular mechanisms and clinical applications of angiogenesis." Nature 473(7347): 298-307.

Cho, H., J. H. Seo, K. H. Wong, Y. Terasaki, J. Park, K. Bong, K. Arai, E. H. Lo and D. Irimia (2015). "Three-Dimensional Blood-Brain Barrier Model for in vitro Studies of Neurovascular Pathology." Sci Rep 5: 15222.

Colgan, O. C., N. T. Collins, G. Ferguson, R. P. Murphy, Y. A. Birney, P. A. Cahill and P. M. Cummins (2008). "Influence of basolateral condition on the regulation of brain microvascular endothelial tight junction properties and barrier function." Brain Res 1193: 84-92.

Colgan, O. C., G. Ferguson, N. T. Collins, R. P. Murphy, G. Meade, P. A. Cahill and P. M. Cummins (2007). "Regulation of bovine brain microvascular endothelial tight 
junction assembly and barrier function by laminar shear stress." Am J Physiol Heart Circ Physiol 292(6): H3190-3197.

Collins, N. T., P. M. Cummins, O. C. Colgan, G. Ferguson, Y. A. Birney, R. P. Murphy, G. Meade and P. A. Cahill (2006). "Cyclic strain-mediated regulation of vascular endothelial occludin and ZO-1: influence on intercellular tight junction assembly and function." Arterioscler Thromb Vasc Biol 26(1): 62-68.

Cucullo, L., M. Hossain, V. Puvenna, N. Marchi and D. Janigro (2011). "The role of shear stress in Blood-Brain Barrier endothelial physiology." BMC Neurosci 12: 40.

Cucullo, L., M. Hossain, W. Tierney and D. Janigro (2013). "A new dynamic in vitro modular capillaries-venules modular system: cerebrovascular physiology in a box." BMC Neurosci 14: 18.

Cucullo, L., M. S. McAllister, K. Kight, L. Krizanac-Bengez, M. Marroni, M. R. Mayberg, K. A. Stanness and D. Janigro (2002). "A new dynamic in vitro model for the multidimensional study of astrocyte-endothelial cell interactions at the bloodbrain barrier." Brain Res 951(2): 243-254.

Czupalla, C. J., S. Liebner and K. Devraj (2014). "In vitro models of the blood-brain barrier." Methods Mol Biol 1135: 415-437. 
Daneman, R., L. Zhou, A. A. Kebede and B. A. Barres (2010). "Pericytes are required for blood-brain barrier integrity during embryogenesis." Nature 468(7323): 562-566.

de Boer, A. G. and P. J. Gaillard (2006). "Blood-brain barrier dysfunction and recovery." J Neural Transm (Vienna) 113(4): 455-462.

Deosarkar, S. P., B. Prabhakarpandian, B. Wang, J. B. Sheffield, B. Krynska and M. F. Kiani (2015). "A Novel Dynamic Neonatal Blood-Brain Barrier on a Chip." PLoS One 10(11): e0142725.

Eichler, A. F., E. Chung, D. P. Kodack, J. S. Loeffler, D. Fukumura and R. K. Jain (2011). "The biology of brain metastases-translation to new therapies." Nat Rev Clin Oncol 8(6): 344-356.

Fan, Y., D. T. Nguyen, Y. Akay, F. Xu and M. Akay (2016). "Engineering a Brain Cancer Chip for High-throughput Drug Screening." Sci Rep 6: 25062.

Folkman, J. (2007). "Angiogenesis: an organizing principle for drug discovery?" Nat Rev Drug Discov 6(4): 273-286.

Frentzas, S., E. Simoneau, V. L. Bridgeman, P. B. Vermeulen, S. Foo, E. Kostaras, M. R. Nathan, A. Wotherspoon, Z. H. Gao, Y. Shi, G. Van den Eynden, F. Daley, C. Peckitt, X. Tan, A. Salman, A. Lazaris, P. Gazinska, T. J. Berg, Z. Eltahir, L. Ritsma, J. van Rheenen, A. Khashper, G. Brown, H. Nystrom, M. Sund, S. Van Laere, E. 
Loyer, L. Dirix, D. Cunningham, P. Metrakos and A. R. Reynolds (2016). "Vessel cooption mediates resistance to anti-angiogenic therapy in liver metastases." Nat Med.

Gaillard, P. J., L. H. Voorwinden, J. L. Nielsen, A. Ivanov, R. Atsumi, H. Engman, C. Ringbom, A. G. de Boer and D. D. Breimer (2001). "Establishment and functional characterization of an in vitro model of the blood-brain barrier, comprising a coculture of brain capillary endothelial cells and astrocytes." Eur J Pharm Sci 12(3): 215-222.

Griep, L. M., F. Wolbers, B. de Wagenaar, P. M. ter Braak, B. B. Weksler, I. A. Romero, P. O. Couraud, I. Vermes, A. D. van der Meer and A. van den Berg (2013). "BBB on chip: microfluidic platform to mechanically and biochemically modulate blood-brain barrier function." Biomed Microdevices 15(1): 145-150.

Hayashi, K., S. Nakao, R. Nakaoke, S. Nakagawa, N. Kitagawa and M. Niwa (2004). "Effects of hypoxia on endothelial/pericytic co-culture model of the blood-brain barrier." Regul Pept 123(1-3): 77-83.

Helms, H. C., N. J. Abbott, M. Burek, R. Cecchelli, P. O. Couraud, M. A. Deli, C. Forster, H. J. Galla, I. A. Romero, E. V. Shusta, M. J. Stebbins, E. Vandenhaute, B. Weksler and B. Brodin (2016). "In vitro models of the blood-brain barrier: An overview of commonly used brain endothelial cell culture models and guidelines for their use." J Cereb Blood Flow Metab 36(5): 862-890. 
Herland, A., A. D. van der Meer, E. A. FitzGerald, T. E. Park, J. J. Sleeboom and D. E. Ingber (2016). "Distinct Contributions of Astrocytes and Pericytes to Neuroinflammation Identified in a 3D Human Blood-Brain Barrier on a Chip." PLoS One 11(3): e0150360.

Janzer, R. C. and M. C. Raff (1987). "Astrocytes induce blood-brain barrier properties in endothelial cells." Nature 325(6101): 253-257.

Kim, J. A., N. D. Tran, Z. Li, F. Yang, W. Zhou and M. J. Fisher (2006). "Brain endothelial hemostasis regulation by pericytes." J Cereb Blood Flow Metab 26(2): 209-217.

Krishna Priya, S., R. P. Nagare, V. S. Sneha, C. Sidhanth, S. Bindhya, P. Manasa and T. S. Ganesan (2016). "Tumour angiogenesis-Origin of blood vessels." Int J Cancer 139(4): 729-735.

Lippmann, E. S., S. M. Azarin, J. E. Kay, R. A. Nessler, H. K. Wilson, A. Al-Ahmad, S. P. Palecek and E. V. Shusta (2012). "Derivation of blood-brain barrier endothelial cells from human pluripotent stem cells." Nat Biotechnol 30(8): 783-791.

Lockman, P. R., R. K. Mittapalli, K. S. Taskar, V. Rudraraju, B. Gril, K. A. Bohn, C. E. Adkins, A. Roberts, H. R. Thorsheim, J. A. Gaasch, S. Huang, D. Palmieri, P. S. Steeg and Q. R. Smith (2010). "Heterogeneous blood-tumor barrier permeability 
determines drug efficacy in experimental brain metastases of breast cancer." Clin Cancer Res 16(23): 5664-5678.

Mager, I., A. H. Meyer, J. Li, M. Lenter, T. Hildebrandt, G. Leparc and M. J. Wood (2016). "Targeting blood-brain-barrier transcytosis - perspectives for drug delivery." Neuropharmacology.

Minami, M. (2011). "[Neuro-glio-vascular interaction in ischemic brains]." Yakugaku Zasshi 131(4): 539-544.

Nakagawa, S., M. A. Deli, H. Kawaguchi, T. Shimizudani, T. Shimono, A. Kittel, K. Tanaka and M. Niwa (2009). "A new blood-brain barrier model using primary rat brain endothelial cells, pericytes and astrocytes." Neurochem Int 54(3-4): 253-263.

Nakagawa, S., M. A. Deli, S. Nakao, M. Honda, K. Hayashi, R. Nakaoke, Y. Kataoka and M. Niwa (2007). "Pericytes from brain microvessels strengthen the barrier integrity in primary cultures of rat brain endothelial cells." Cell Mol Neurobiol 27(6): 687-694.

Noseda, M., L. Chang, G. McLean, J. E. Grim, B. E. Clurman, L. L. Smith and A. Karsan (2004). "Notch activation induces endothelial cell cycle arrest and participates in contact inhibition: role of p21Cip1 repression." Mol Cell Biol 24(20): 8813-8822. 
Pardridge, W. M. (1999). "Blood-brain barrier biology and methodology." Neurovirol 5(6): 556-569.

Patabendige, A., R. A. Skinner and N. J. Abbott (2013). "Establishment of a simplified in vitro porcine blood-brain barrier model with high transendothelial electrical resistance." Brain Res 1521: 1-15.

Perel, P., I. Roberts, E. Sena, P. Wheble, C. Briscoe, P. Sandercock, M. Macleod, L. E. Mignini, P. Jayaram and K. S. Khan (2007). "Comparison of treatment effects between animal experiments and clinical trials: systematic review." BMJ 334(7586): 197.

Petty, M. A. and E. H. Lo (2002). "Junctional complexes of the blood-brain barrier: permeability changes in neuroinflammation." Prog Neurobiol 68(5): 311-323.

Prabhakarpandian, B., M. C. Shen, J. B. Nichols, I. R. Mills, M. SidorykWegrzynowicz, M. Aschner and K. Pant (2013). "SyM-BBB: a microfluidic Blood Brain Barrier model." Lab Chip 13(6): 1093-1101.

Roberts, L. M., D. S. Black, C. Raman, K. Woodford, M. Zhou, J. E. Haggerty, A. T. Yan, S. E. Cwirla and K. K. Grindstaff (2008). "Subcellular localization of transporters along the rat blood-brain barrier and blood-cerebral-spinal fluid barrier by in vivo biotinylation." Neuroscience 155(2): 423-438. 
Santaguida, S., D. Janigro, M. Hossain, E. Oby, E. Rapp and L. Cucullo (2006). "Side by side comparison between dynamic versus static models of blood-brain barrier in vitro: a permeability study." Brain Res 1109(1): 1-13.

Schinkel, A. H. (1999). "P-Glycoprotein, a gatekeeper in the blood-brain barrier." Adv Drug Deliv Rev 36(2-3): 179-194.

Sellgren, K. L., B. T. Hawkins and S. Grego (2015). "An optically transparent membrane supports shear stress studies in a three-dimensional microfluidic neurovascular unit model." Biomicrofluidics 9(6): 061102.

Siddharthan, V., Y. V. Kim, S. Liu and K. S. Kim (2007). "Human astrocytes/astrocyte-conditioned medium and shear stress enhance the barrier properties of human brain microvascular endothelial cells." Brain Res 1147: 39-50.

Srinivasan, B., A. R. Kolli, M. B. Esch, H. E. Abaci, M. L. Shuler and J. J. Hickman (2015). "TEER measurement techniques for in vitro barrier model systems." J Lab Autom 20(2): 107-126.

Stanimirovic, D. B., M. Bani-Yaghoub, M. Perkins and A. S. Haqqani (2015). "Blood-brain barrier models: in vitro to in vivo translation in preclinical development of CNS-targeting biotherapeutics." Expert Opin Drug Discov 10(2): 141-155. 
Stanness, K. A., E. Guatteo and D. Janigro (1996). "A dynamic model of the bloodbrain barrier "in vitro"." Neurotoxicology 17(2): 481-496.

Sumi, N., T. Nishioku, F. Takata, J. Matsumoto, T. Watanabe, H. Shuto, A. Yamauchi, S. Dohgu and Y. Kataoka (2010). "Lipopolysaccharide-activated microglia induce dysfunction of the blood-brain barrier in rat microvascular endothelial cells co-cultured with microglia." Cell Mol Neurobiol 30(2): 247-253.

Taber, K. H. and R. A. Hurley (2008). "Astroglia: not just glue." J Neuropsychiatry Clin Neurosci 20(2): iv-129.

Talmadge, J. E. and I. J. Fidler (2010). "AACR centennial series: the biology of cancer metastasis: historical perspective." Cancer Res 70(14): 5649-5669.

Tarbell, J. M. (2010). "Shear stress and the endothelial transport barrier." Cardiovasc Res 87(2): 320-330.

Thomsen, L. B., A. Burkhart and T. Moos (2015). "A Triple Culture Model of the Blood-Brain Barrier Using Porcine Brain Endothelial cells, Astrocytes and Pericytes." PLoS One 10(8): e0134765.

van der Helm, M. W., A. D. van der Meer, J. C. Eijkel, A. van den Berg and L. I. Segerink (2016). "Microfluidic organ-on-chip technology for blood-brain barrier research." Tissue Barriers 4(1): e1142493. 
Vorbrodt, A. W. and D. H. Dobrogowska (2003). "Molecular anatomy of intercellular junctions in brain endothelial and epithelial barriers: electron microscopist's view." Brain Res Brain Res Rev 42(3): 221-242.

Wang, J. D., S. Khafagy el, K. Khanafer, S. Takayama and M. E. ElSayed (2016). "Organization of Endothelial Cells, Pericytes, and Astrocytes into a 3D Microfluidic in Vitro Model of the Blood-Brain Barrier." Mol Pharm 13(3): 895-906.

Wolff, A., M. Antfolk, B. Brodin and M. Tenje (2015). "In Vitro Blood-Brain Barrier Models-An Overview of Established Models and New Microfluidic Approaches." $\underline{\mathrm{J}}$ Pharm Sci 104(9): 2727-2746.

Wong, A. D., M. Ye, A. F. Levy, J. D. Rothstein, D. E. Bergles and P. C. Searson (2013). "The blood-brain barrier: an engineering perspective." Front Neuroeng 6: 7.

Xue, Q., Y. Liu, H. Qi, Q. Ma, L. Xu, W. Chen, G. Chen and X. Xu (2013). "A novel brain neurovascular unit model with neurons, astrocytes and microvascular endothelial cells of rat." Int J Biol Sci 9(2): 174-189.

Yeon, J. H., D. Na, K. Choi, S. W. Ryu, C. Choi and J. K. Park (2012). "Reliable permeability assay system in a microfluidic device mimicking cerebral vasculatures." Biomed Microdevices 14(6): 1141-1148. 
Zheng, W., M. Aschner and J. F. Ghersi-Egea (2003). "Brain barrier systems: a new frontier in metal neurotoxicological research." Toxicol Appl Pharmacol 192(1): 1-11. 
CHAPTER 3

PERMEABILITY ACROSS THE BLOOD-BRAIN BARRIER AND BLOOD-TUMOR BARRIER; A NOVEL IN VITRO MODEL ON A CHIP

\subsection{Introduction}

The occurrence of brain metastases in breast cancer patients is approximately 10\%-16\% (Lin, Amiri-Kordestani et al. 2013). Due to improvements in chemotherapy the overall survival of breast cancer patients has increased. Unfortunately with prolonged survival the incidence of patients developing symptomatic brain metastases has increased. One of the leading complications of brain metastases is the inability of drugs to reach the tumor at dosage levels equivalent to adequately induce cytotoxicity. This is due, in part, to the presence of a partially intact blood-brain barrier (BBB).

\subsubsection{The Blood-Brain Barrier}

The BBB is a complex anatomical network, functioning to strictly regulate the movement of molecules, and ions from the blood to the brain, and back. In addition, the BBB serves as the conduit to supply the brain with the essential nutrients it needs, while facilitating the excretion of waste products through efflux (Abbott, Ronnback et al. 2006, Daneman and Prat 2015). The hallmark of the BBB is the presence of endothelial cells that are tightly connected by tight junction protein complexes, composed of claudins, 
occludins, and junction adhesion molecules (Serlin, Shelef et al. 2015). In addition to endothelia, the BBB has a thick basal membrane with pericytes and astrocytic foot processes in close proximity (Golden and Pardridge 1999, Pardridge 2005). The net effect of this anatomical structure results in the transendothelial electrical resistance (TEER) of brain capillaries being $2,000 \mathrm{ohm} * \mathrm{~cm}^{2}$, in comparison to $2-20 \mathrm{ohm}^{*} \mathrm{~cm}^{2}$ in peripheral capillaries (Crone and Christensen 1981, Olesen and Crone 1983). In addition to the structural components, the BBB his highly enriched in efflux transporters that actively restrict the entry a large and diverse set of lipophilic solutes from accumulating in the brain (Loscher and Potschka 2005, Loscher and Potschka 2005, Shen and Zhang 2010, Adkins, Mittapalli et al. 2013).

\subsubsection{The Blood-Tumor Barrier}

When metastatic cancer cells invade the central nervous system (CNS) they may eventually colonize and begin to proliferate into a larger tumor mass. Once the lesion has grown to a point that it has areas of hypoxia, the tumor will secrete high amounts of vascular endothelial growth factor in an attempt to develop a new blood supply (Folkman 1971, Ferrara and Davis-Smyth 1997, Neufeld, Cohen et al. 1999, Plate, Scholz et al. 2012). This vasculature (blood-tumor barrier; BTB) is different than the BBB predominantly because the astrocytes, pericytes and neurons are no longer in close proximity to the capillary. It is hypothesized that these anatomical changes result in vasculature that has greater permeability than the BBB (Henson, Cordon-Cardo et al. 1992, Hobbs, Monsky et al. 1998, Liebner, Fischmann et al. 2000, Abbott, Ronnback et al. 2006, Deo, Theil et al. 2013). The BTB may also have a somewhat different and 
varied expression of efflux transporters depending on the CNS malignancy (Sawada, Kato et al. 1999, Tews, Nissen et al. 2000, Demeule, Shedid et al. 2001, Lockman, Mittapalli et al. 2010). Despite the apparent breakdown of the BBB in the presence of a tumor, the BTB still limits drug movement in to the CNS lesion substantially greater than in peripheral tumors.

\subsubsection{In vitro vs in vivo BBB and BTB Models}

Currently, there are no widely validated in vitro models of the BTB. The most widely used in vitro BBB model, that has been somewhat used to model the BTB, is a transwell insert system. Briefly, the model consists of upper chamber with endothelial cells grown on the surface separated from a lower chamber that may or may not have astrocytes and or cancer cells grown in separated by a porous membrane (Bicker, Alves et al. 2014, Czupalla, Liebner et al. 2014, Srinivasan, Kolli et al. 2015, Helms, Abbott et al. 2016). Drug movement is modeled by measuring accumulation in the lower chamber versus time. The transwell model has limitations. First, there is a lack of flow exerted on the endothelia resulting in poor cell morphology and a "leakier" barrier compared to in vivo data (Cucullo, Hossain et al. 2011, Prabhakarpandian, Shen et al. 2013, Czupalla, Liebner et al. 2014, Deosarkar, Prabhakarpandian et al. 2015, Helms, Abbott et al. 2016). Second, endothelial cells do not uniformly attach to the outer side of the insert, leaving gaps between the endothelial cells and the edge of the insert also resulting in increased permeability (Santaguida, Janigro et al. 2006). Third, an unstirred water later forms on the surface of the endothelia which results in increased permeability for hydrophilic drugs and decreased permeability for lipid soluble drugs (Barry and Diamond 1984, Korjamo, 
Heikkinen et al. 2008, Korjamo, Heikkinen et al. 2009, Loftsson 2012, Ghosh, Scott et al. 2014).

Herein we characterize novel in vitro microfluidic models of the BTB and BBB using a co-culture of endothelial cells and astrocytes along with tumor cells. This model incorporates flow during culture of the endothelia and has a micro-tubular lumen, which in other work has substantially reduced limitations seen in transwells (Neuhaus, Lauer et al. 2006, Cucullo, Marchi et al. 2011, Booth and Kim 2012, Griep, Wolbers et al. 2013, Herland, van der Meer et al. 2016). This model is unique from other flow based models in that it allows for a co-culture or triple culture of relevant cells, it is easily duplicated, it is commercially available and provides a cost-effective solution for running multiple and parallel assays.

\subsection{Materials and Methods}

\subsubsection{Microfluidic Device}

Co-Culture idealized microvascular networks used in this study were obtained from SynVivo Inc. Huntsville, AL. The device consists of a central compartment (basolateral) that is comprised of the brain tissue cells (astrocytes, pericytes, neurons) and the outer compartment (apical) that is comprised of the endothelial cells and provides perfusion similar to physiological fluid flow conditions. The outer compartments and central compartment are separated by an interface with a series of $3 \mu \mathrm{m}$ pores along the length, replacing the use of membranes in conventional models. 


\subsubsection{Chemicals}

Sulforhodamine 101 Acid Chloride (Free TRD), Rhodamine-123 (Rho123), Texas Red 3000 MW Dextran (TRD 3 kDa) and Texas Red 70,000 MW Dextran (TRD 70 kDa) were purchased from ThermoFisher Scientific (Grand Island, NY). Verapamil was purchased from Sigma (St. Louis, MO). Cyclosporine A was purchased from Toronto Research Chemicals Inc. (Toronto, Canada). All other chemicals used were of analytical grade and were used as supplied.

\subsubsection{Cell Culture}

Human Umbilical Vein Endothelial Cells (HUVECs) were purchased from Lonza (Allendale, NJ). CTX-TDR2 rat brain astrocyte cell line was kindly donated by Dr. Jim Simpkins (West Virginia University, Morgantown, WV). Met-1 murine metastatic breast cancer cells were a kind gift from Dr. Alexander Borowsky (UC Davis, Sacramento, CA). All cells were maintained in Endothelial Basal Medium - 2 (EBM-2) supplemented with the EGM-2 BulletKit from Lonza (Allendale, NJ). Cells were grown in a $37^{\circ} \mathrm{C}$ humidified incubator with $5 \% \mathrm{CO}_{2}$ until $\sim 85 \%$ confluent.

\subsubsection{Cell Culture in Microfluidic Chip}

Matrigel (40ug/cm2, EMD Milipore, Billerica MA) was injected into the central compartment and allowed to sit covered in ice for approximately 1 hour, after which serum-free media was promptly injected to wash the central compartment. Fibronectin (200 $\mu \mathrm{g} \mathrm{mL}^{-1}$, EMD Milipore, Billerica MA) was then injected in one of the outer sides of the device and allowed to incubate at $37^{\circ} \mathrm{C}$ overnight. Prior to the seeding of all cells, 
the device was flushed with EBM-2 media. Astrocytes/Met-1 cells were harvested using TrypLE Select (ThermoFisher, Waltham MA) and re-suspended into a final concentration of $\sim 1 \times 10^{7} \mathrm{~mL}^{-1}$ cells for injection, and were seeded at a flow rate of $10 \mu \mathrm{L} / \mathrm{min}$ in the central compartment using a Pump 11 Elite Nanomite programmable syringe pump (Harvard Apparatus, Holliston MA). The inlet port tubing was clamped when cells reached an intra-central compartment density of $\sim 50 \%$ and chip was transferred to a $\mathrm{CO}_{2}$ incubator at $37^{\circ} \mathrm{C}$ and allowed to attach for 2 hours. HUVECs were harvested using TrypLE Select (ThermoFisher, Waltham MA) in the same process as described above,

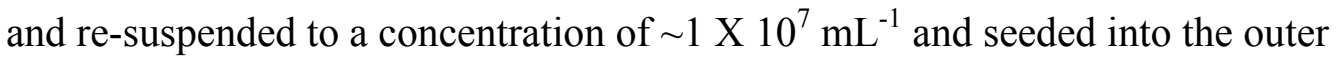
compartment previously coated with Fibronectin at a flow rate of $6 \mu \mathrm{L} / \mathrm{min}$ using a Pump 11 Elite Nanomite programmable syringe pump (Harvard Apparatus, Holliston MA). Inlet port tubing was clamped when HUVECs reached an intra-outer compartment density of $\sim 90 \%$, then chip was transferred to a $\mathrm{CO}_{2}$ incubator at $37^{\circ} \mathrm{C}$ and allowed to attach for 24 hours. After 6 hours of incubation, medium in central and both outer compartments was replaced with fresh EBM-2 medium and repeated again at 24 hours. Astrocyte/Met-1 cells were maintained in the central compartment under static conditions in EBM-2 medium while EBM-2 medium was prepared in syringes mounted on a programmable PHD 2000 syringe pump (Harvard Apparatus, Holliston MA), connected to the chips through $\sim 12$ inches of sterile Tygon tubing (Harvard Apparatus, Holliston MA). This medium was flowed at a flow rate of $0.02 \mu \mathrm{L} /$ min over the seeded HUVECs in the outer compartment for 4 hours, then increased to $0.05 \mu \mathrm{L} / \mathrm{min}$ after 4 hours, and finally to $0.1 \mu \mathrm{L} /$ min after 4 more hours, maintaining a flow of $0.1 \mu \mathrm{L} / \mathrm{min}$ for 24 hours. 


\subsubsection{In vitro transport studies}

EBM-2 Medium was incubated in a BD Luer-Lok Syringe with either Free TRD (600 mg/mL), TRD $3 \mathrm{kDa}(600 \mathrm{mg} / \mathrm{mL})$, TRD $70 \mathrm{kDa}(600 \mathrm{mg} / \mathrm{mL})$, or incubated with Rho123 (600 mg/mL) in the presence or absence of known P-glycoprotein (p-gp) inhibitors (verapamil: $50 \mathrm{mM}$, cyclosporine A: $10 \mathrm{mM}$ ) and mounted on a programmable PHD 2000 syringe pump (Harvard Apparatus, Holliston MA), with syringes connected to the chips through sterile Tygon tubing (Harvard Apparatus, Holliston MA). Permeability was measured through the injection of desired tracer into the outer compartment at 0.1 $\mu \mathrm{L} /$ min for a total of 90 minutes while brightfield images (acquired at a $25 \mathrm{~ms}$ exposure) and fluorescent images (acquired at a $200 \mathrm{~ms}$ exposure) were acquired every 2 minutes. Permeability of each tracer was determined using NIS Elements Imaging Software. Using linear regression (Prism 6.0), the slope of the best-fit line was used to represent the relative $\mathrm{k}_{\mathrm{in}}$, or rate of accumulation, of fluorescence in the central compartment (comparable to the concentration of drug found in normal brain) divided by the accumulation of fluorescence in the outer compartment (comparable to the concentration of drug found in the plasma of the BBB vasculature). Unless otherwise stated, data are presented as mean \pm S.E.M.

\subsubsection{Quantification of fluorescent tracers using fluorescent microscopy}

Chips were mounted on an automated stage enclosure, maintained at $37^{\circ} \mathrm{C}$ with 5\% $\mathrm{CO}_{2}$, on a Nikon Eclipse TE2000-E Live Cell Sweptfield Confocal microscope (Melville, NY). Acquisition of images and fluorescence was achieved through the utilization of a Photometrics CoolSnap HQ2 Monochrome CCD Camera (Tucson, AZ) 
with a 20x/0.75 Plan Fluor Phase Contrast objective with a total field of $6 \mathrm{X} 8$, stitching images using brightfield with a $10 \%$ overlay. Brightfield and fluorescent images were taken every two minutes for 90 minutes. Excitation and emission of Free Texas Red, Texas Red $3 \mathrm{kDa}$ and $70 \mathrm{kDa}$, was obtained using the TRITC epiflourescence filter (peak fluorophore excitation is $596 \mathrm{~nm}$ and emission is $615 \mathrm{~nm}$ ); excitation filter wheel of $555 / 25 x$, emission filter wheel of $605 / 52 \mathrm{~m}$ and dichromatic mirror at 89000 sedat quad. The excitation and emission of Rho123 (+/- cyclosporine A or verapamil) was obtained using the FITC epiflourescence filter (peak fluorophore excitation is $511 \mathrm{~nm}$ and emission is $534 \mathrm{~nm}$ ); excitation filter wheel of 490/20x, emission filter wheel of 525/36m and dichromatic mirror at 89000 sedat quad.

\subsubsection{Kinetic analysis}

Unidirectional uptake transfer constants $\left(\mathrm{k}_{\text {in }}\right)$ were calculated from the following relationship to the linear portion of the uptake curve:

$$
\left(\mathrm{C}_{\mathrm{CC}}+\mathrm{C}_{\mathrm{PF}}\right) / \mathrm{C}_{\mathrm{PF}}=\mathrm{k}_{\text {in }}(\mathrm{t})+\mathrm{O}_{\mathrm{C}}
$$

(Equation 3.1)

Where $\mathrm{C}_{\mathrm{CC}}$ is the sum intensity of fluorophore in the region of interest in the central compartment $(\mathrm{au})$ at the end of perfusion, $\mathrm{C}_{\mathrm{PF}}$ is the sum intensity of fluorophore $(\mathrm{au})$ in the region of interest within the outer compartment, $t$ is the perfusion time in minutes from the time the device reached steady state, and $\mathrm{O}_{\mathrm{C}}$ is the calculated intercept $(\mathrm{T}=0$ min; "outer compartment volume" (au)). Since the device took 22 minutes to reach steady state, $\mathrm{t}=0$ minutes is 22 minutes after start of the experiment, but 0 minutes from the start of steady state. After the determination of a perfusion time where an adequate amount of fluorescent marker was allowed to pass into brain, while still remaining in the linear 
uptake zone, $\mathrm{k}_{\text {in }}$ was determined (Takasato, Rapoport et al. 1984, Smith and Takasato 1986).

\subsubsection{Statistical Analysis}

The slope of the line $\left(\mathrm{k}_{\mathrm{in}}\right)$ was determined with linear regression using best-fit values. One-way ANOVA analysis and unpaired t test with Welch's correction, followed by an $\mathrm{F}$ test to compare variances were used for the comparison of the $\mathrm{k}_{\text {in }}$ values between unrestricted diffusion, BBB, and BTB among each tracer and with Rho123 in absence and presence of inhibitors. For all data, errors are reported as standard error of the mean unless otherwise indicated. Differences were considered statistically significant at the $\mathrm{p}<$ 0.05 level. (GraphPad Prism version 6.00 for Mac, GraphPad Software, San Diego, CA, USA).

\subsection{Results}

In this study we evaluate BBB and BTB transfer rates of Free TRD, Texas Red 3 $\mathrm{kDa}$, Texas Red $70 \mathrm{kDa}$, and Rho123 (with and without inhibitors) (Fig 3.1) in a novel microfluidic $\mathrm{BBB}$ and $\mathrm{BTB}$ model as validation to previously published literature (Mittapalli, Manda et al. 2013). Briefly in this model, endothelial cells are seeded in the outer compartments, while astrocytes (BBB) or brain seeding breast cancer cells (BTB) are seeded in the central compartment. The porous architecture between the two compartments allows for cellular crosstalk and biochemical exchanges, while shear stress from perfusate flow facilitates development of endothelial morphology (Deosarkar, Prabhakarpandian et al. 2015). Confocal brightfield images show the differences in 
morphology between endothelial cells with and without flow (Fig 3.1B-C). In order to verify a confluent $360^{\circ}$ coating of endothelial cells within the outer compartment, we used a Nikon A1R Confocal on Eclipse TiE Microscope to acquire a 3D z-stack of the outer compartment. Utilizing this system, DAPI stained endothelial cells were imaged from the bottom (Fig 3.2A), through to the top (Fig 3.2B) showing HUVECs wrapping around the sides of the outer compartment (Fig 3.2C) connecting the HUVECs on the top to the HUVECs on the bottom, verifying confluent formation of a tubular in vitro microvasculature.

In initial experiments, we determined unrestricted diffusion rates of different sized molecules, by perfusing solutes through microfluidic chips without endothelial cells or astrocytes/cancer cells. To quantify tracer accumulation, regions of interest were selected to determine sum fluorescence intensity in the outer compartment (ROI 136), central compartment (ROI 139), and background (ROI 165) over time (3.1D). ROI 165 was taken to ensure data received in the outer and central compartments were significant when compared to the background sum fluorescence. We observed (Fig. 3.3) that small tracers $(<1000 \mathrm{Da})$ had a diffusion rate of $22.8 \pm 2.5 \times 10^{-3}, \mathrm{n}=6$, which was not significantly different compared to tracers of molecular weights between $3-5 \mathrm{kDa}(22.1 \pm$ $\left.8.5 \times 10^{-3}, \mathrm{n}=3\right)$ and $>60 \mathrm{kDa}\left(17.5 \pm 4.2 \times 10^{-3}, \mathrm{n}=3\right)$.

In our next experiments, we qualitatively imaged Texas Red accumulation from 0 - 90 min in the BBB model (Fig 3.4A-3.4D). Linear accumulation of the dye in the central chamber of the BBB model is quantitatively shown in Figure 3.4E. We then 
determined $\mathrm{k}_{\text {in }}$ values for each tracer in both the BBB and BTB model (Equation 3.1). Free Texas Red $\mathrm{k}_{\text {in }}$ values $\left(\right.$ Fig 3.5A) for the BBB $\left(2.5 \pm 0.3 \times 10^{-3}, \mathrm{n}=6\right)$ and BTB $(13.1$ $\left.\pm 1.3 \times 10^{-3}, \mathrm{n}=4\right)$ were significantly different $(\mathrm{p}<0.05)$ between each other. Texas Red $3 \mathrm{kDa}$ values (Fig 3.5B) for the BBB $\left(0.1 \pm 0.1 \times 10^{-3}, \mathrm{n}=3\right)$ and BTB $\left(1.8 \pm 1.0 \times 10^{-3}\right.$, $\mathrm{n}=3)$ and Texas Red $70 \mathrm{kDa}$ values (Fig 3.5C) for the BBB $\left(1.1 \pm 0.9 \times 10^{-3}, \mathrm{n}=3\right)$ and BTB $\left(4.5 \pm 2.4 \times 10^{-3}, \mathrm{n}=3\right)$ were also significant $(\mathrm{p}<0.05)$ when compared to the unrestricted diffusion $\mathrm{k}_{\text {in }}$ but significance was not observed between the BBB and BTB models of these dyes.

To determine if $\mathrm{p}$-gp inhibitors alter the accumulation of $\mathrm{p}$-gp sensitive fluorescent dye accumulation into the central compartment we perfused Rho123 in the absence and presence of p-gp inhibitors cyclosporine A (10 mM), and verapamil (50mM) (Mittapalli, Manda et al. 2013). We qualitatively observed an increase in dye accumulation in the central compartment over the course of 90 minutes in both the BBB (Fig 3.6A) and BTB (Fig 3.6B) models (Fig 3.6C). Quantitatively, we observed a 14fold increase of Rho123 in the central compartment, in the presence of p-gp inhibitor verapamil $\left(14.7 \pm 7.5 \times 10^{-3}, n=3\right)$, and a significant $(\mathrm{p}<0.05)$ eight fold increase of Rho123 with cyclosporine A $\left(8.8 \pm 1.8 \times 10^{-3}, \mathrm{n}=3\right)$ when compared to control Rho123 $\left(0.6 \pm 0.1 \times 10^{-3}, \mathrm{n}=4\right)$ in the BBB model (Fig 3.6D). Similarly in the BTB model, a three-fold increase was observed in Rhodamine-123 permeability in the presence of $\mathrm{p}$-gp inhibitor verapamil $\left(10.3 \pm 3.1 \times 10^{-3}, \mathrm{n}=3\right)$, and a two-fold increase with cyclosporine A $\left(7.1 \pm 5.2 \times 10^{-3}, \mathrm{n}=3\right)$ when compared to Rho123 control $\left(3.2 \pm 2.8 \times 10^{-3}, \mathrm{n}=3\right)($ Fig 3.6E). 


\subsection{Discussion}

The results of the studies presented herein suggest that a novel microfluidic chip in part mimics the in vivo BBB and BTB with regard to passive permeability and efflux (Adkins, Mohammad et al. 2016). Importantly, this study demonstrates that perfusion flow through the luminal compartment improves endothelial function. This model also has potential to be used as in screening assays for drug discovery and development for central nervous system disease.

Predominant in vitro BBB models have some key similarities. First, there is a presence of some type of "barrier" cell in a luminal or outer compartment (representing the vascular lumen). These cells range from primary or immortalized brain endothelial cells (most commonly rat, mouse, or human), peripheral endothelial (HUVECs), or stemcell derived cells (Stanimirovic, Bani-Yaghoub et al. 2015). These barrier cells typically express tight junction proteins, which seal the endothelial cells together and produce higher TEER values (Reese and Karnovsky 1967, Brightman and Reese 1969, Zlokovic 2008). Second the models usually include the presence of a semipermeable basement membrane separating the outer (lumen) and central (brain side) compartments. Lastly, cells, typically astrocytes and or pericytes, are seeded in the central compartment in an effort to mimic the brain microenvironment. The addition of these cells provide cell to cell communication to the endothelial cells in the outer compartment, resulting in the formation of tighter barrier and an increase in TEER (Abbott 2002, Coisne, Dehouck et

al. 2005, Garberg, Ball et al. 2005, Pardridge 2005, Pardridge 2007, Abbott, Patabendige 
et al. 2010, Stanimirovic, Bani-Yaghoub et al. 2015, Helms, Abbott et al. 2016).

Germane to this work, to re-create the BTB, astrocytes and or pericytes are replaced with tumor cells in the central compartment. In vivo, angiogenesis occurs with the establishment of tumor tissue, resulting in the presence of fenestrations, gaps between the endothelial cells, varied expression of efflux transporters, and an increase in permeability (Schlageter, Molnar et al. 1999, Plate, Scholz et al. 2012).

The use of dyes has been a long-standing method to evaluate the integrity of the BBB and the breakdown of the BTB. (Ehrlich 1885, Hawkins and Davis 2005, Hawkins and Egleton 2006, Goldmann 1913). Some of the earliest work using dyes dates back to the the $19^{\text {th }}$ century, where Paul Ehrlich and Edwin Goldmann intravenously injected water-soluble dyes and observed that the dyes did not have the ability to freely exchange between the vascular and brain parenchyma compartment (Ehrlich 1885)'(Goldmann 1913). Dyes have also been used as a tool to visualize and qualitatively measure the disruption at the BBB (Bakay, Ballantine et al. 1956, Schettler and Shealy 1970, da Costa 1972, Nemeroff and Crisley 1975, Lin and Kormano 1977) as well as the BTB (Mittapalli, Manda et al. 2013, Adkins, Mohammad et al. 2016). Passive permeability dyes are a simple way to compare rates of diffusion between different models in vivo and in vitro.

To measure the unrestricted diffusion (the absence of cells) of molecules from the outer chamber to the center chamber, we observed that the diffusion rates $\left(\mathrm{k}_{\mathrm{in}}\right)$, from the outer compartment to the central compartment, of all three sized molecules were not 
significantly different from each other. These data are consistent with previous work showing that if the diameter of each molecule being tested is at least $12 \mathrm{x}$ less than the barrier defects, then diffusion will remain constant for all molecules (Nakagawa, Groothuis et al. 1987).

An interesting aspect of our observations was the similarity of efflux function that existed in the microfluidic model compared to the in vivo BBB (Adkins, Mittapalli et al. 2013). Rhodamine- 123 is subject to p-gp mediated efflux at both the BBB and the BTB. When rho123 and an inhibitor of p-gp are administered concurrently, dye accumulates in brain $\sim 10-12$ fold higher than in the absence of efflux inhibition (Mittapalli, Manda et al. 2013). Similarly, in this work when verapamil or cyclosporine A was added to the outer chamber of the microfluidic device, Rho123 accumulation increased similar to in vivo reports (Adkins, Mittapalli et al. 2013). Further, p-gp function retains function despite barrier breakdown in a number of pathologies (Cordon-Cardo, O'Brien et al. 1990, Adkins, Mittapalli et al. 2013). The data herein agree that the degree of efflux function for the BTB, though disrupted, is intact and it retains the ability to restrict drug and dye movement from the vasculature to the brain compartment.

Transwells are a widely used in vitro method to study the BBB. Transwells are cheap, available in high throughput assays, and easy to use. However, there are substantial limitations. First, transport kinetics in transwell systems are strongly influenced by an unstirred water layer that exists on the outer side of the endothelial cells. The unstirred water layer will decrease the apparent permeability rate of lipid soluble and 
to some extent water-soluble molecules. Second, since the cells are grown in a static media there is no shear stress (or flow) forced on the endothelial cells, which may contribute to the low passive permeability measurements which can be as low as $\sim 74$ $\Omega \cdot \mathrm{cm}^{2}$ (Man, Ubogu et al. 2008), compared to in vivo values of $\sim 2000 \Omega \cdot \mathrm{cm}^{2 \text { (Crone and }}$ Olesen 1982). While a few other in vitro models and microfluidic devices have a flow component (Neuhaus, Lauer et al. 2006, Cucullo, Marchi et al. 2011, Booth and Kim 2012, Griep, Wolbers et al. 2013, Herland, van der Meer et al. 2016) this microfluidic device is the first commercially available blood-tumor barrier using a microfluidic model utilizing brain-seeking cells with shear stress similar to in vivo (Deosarkar, Prabhakarpandian et al. 2015) in addition to real-time visualization and quantitation. 


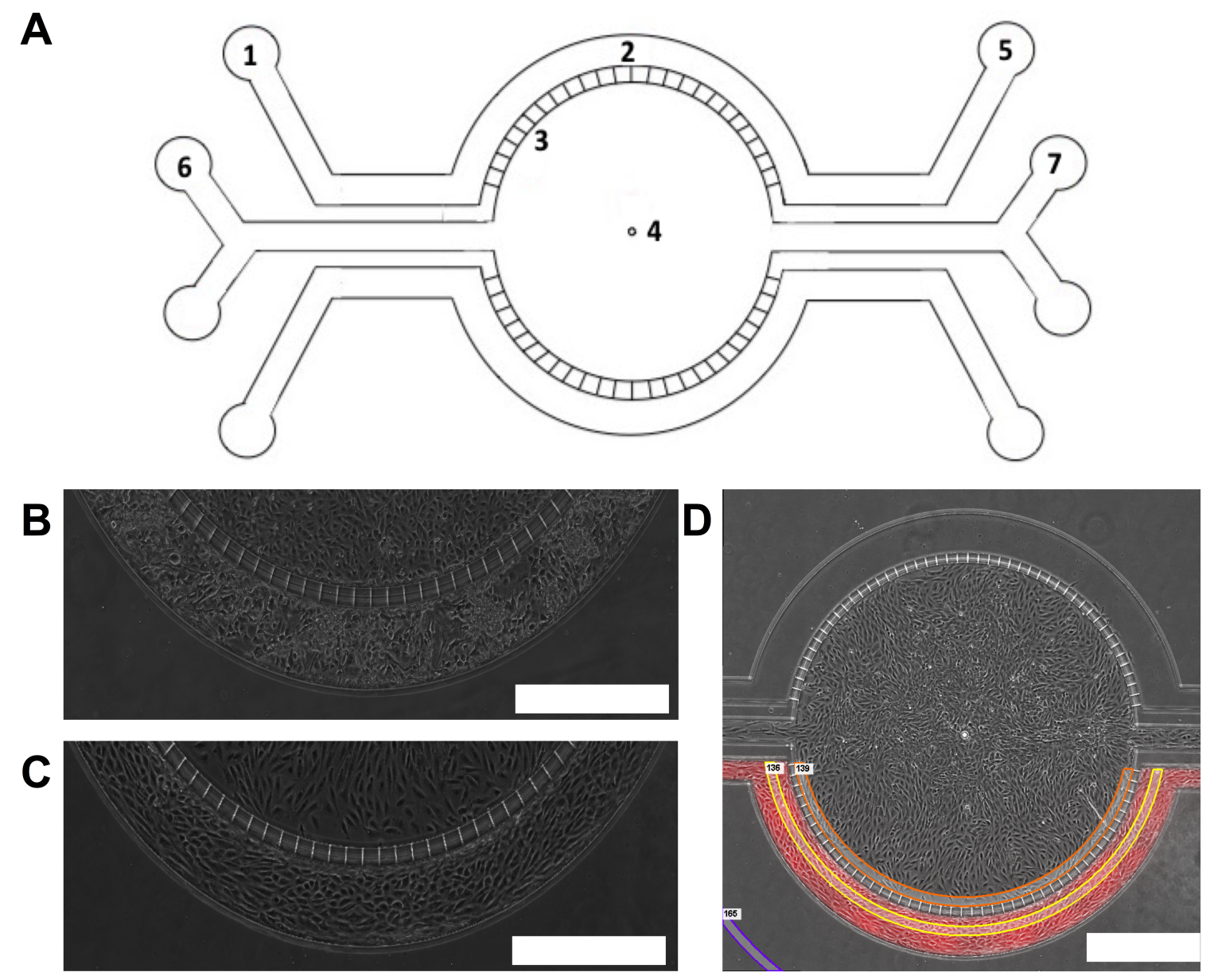

Figure 3.1: Microfluidic chip schematic and methods.

(A) Schematic of the SynVivo BBB Microfluidic Chip: (1) Inlet port where media with or without tracer is flowed through the outer compartment to change media for HUVECs. (2) Outer Compartment, containing HUVECs. (3) $3 \mu \mathrm{m}$ pores, to allow diffusion of media and tracer between the central and outer compartments. (4) Central Compartment, containing astrocytes or cancer cells. (5) Outlet port where perfusate from the outer compartment is collected. (6) Inlet port for central compartment, used to seed 
and change media for the astrocytes/cancer cells in the central compartment. (7) Output ports where perfusate from the central compartment is collected. (B) Morphology of astrocytes in the central compartment and HUVECs in the outer compartment without the addition of flow (C) Morphology of astrocytes in the central compartment and HUVECs in the outer compartment with the addition of flow. (D) Representation of where the regions of interest (ROI) measurements are taken for data analysis. 


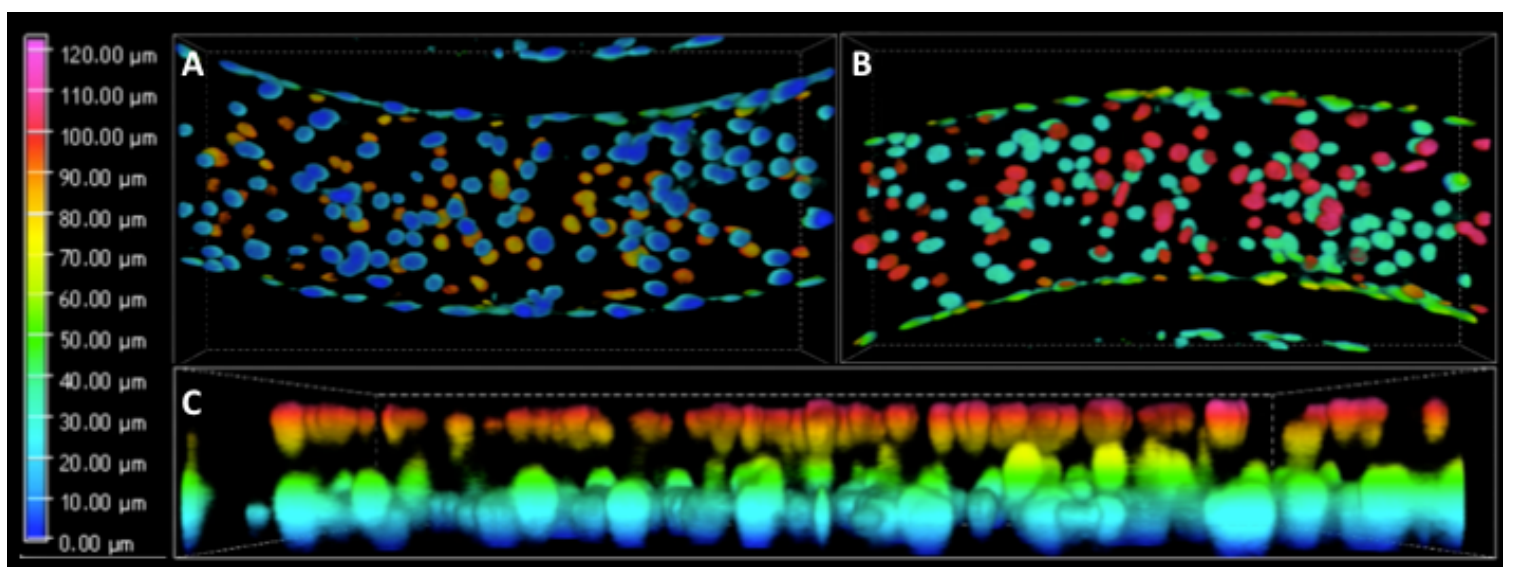

Figure 3.2: 3-D confocal image of DAPI labeled HUVECs in apical chamber.

3-dimensional confocal images of DAPI labeled HUVECs in the outer compartment demonstrating a $360^{\circ}$ coating of cells. The nuclei of the HUVECs are seen on the bottom (A) and top (B) and in a side view (C). 


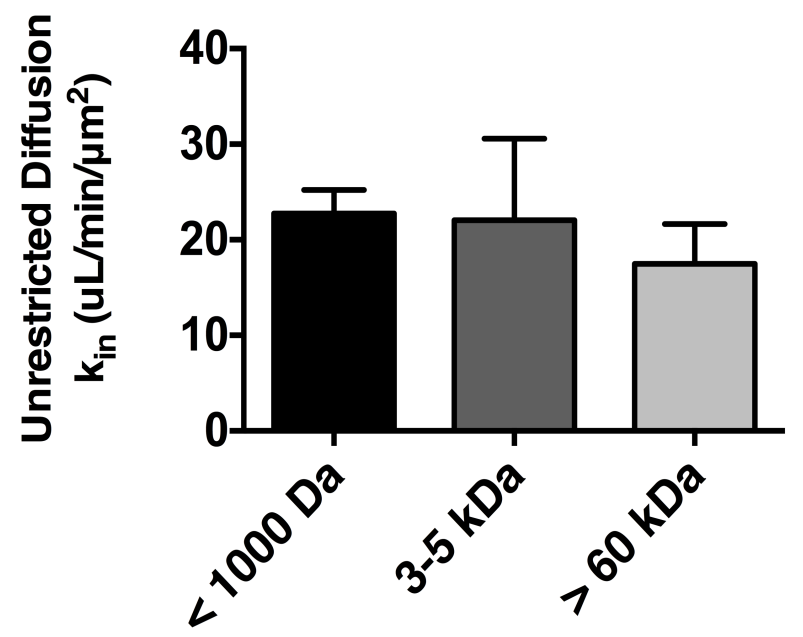

Figure 3.3: Diffusion rates of different sized MW tracers in the same model.

The diffusion rates of free MW tracers $<1000 \mathrm{Da}(\mathbf{A}), 3-5 \mathrm{kDa}(\mathbf{B})$ and $>60 \mathrm{kDa}(\mathbf{C})$ in an unrestricted, cell free microfluidic chips are shown. Statistical significance was determined using one-way ANOVA followed by Tukey's multiple comparison tests, and student's $t$-test; $\mathrm{n}=3-6$ chips. All data represent mean \pm SEM. 


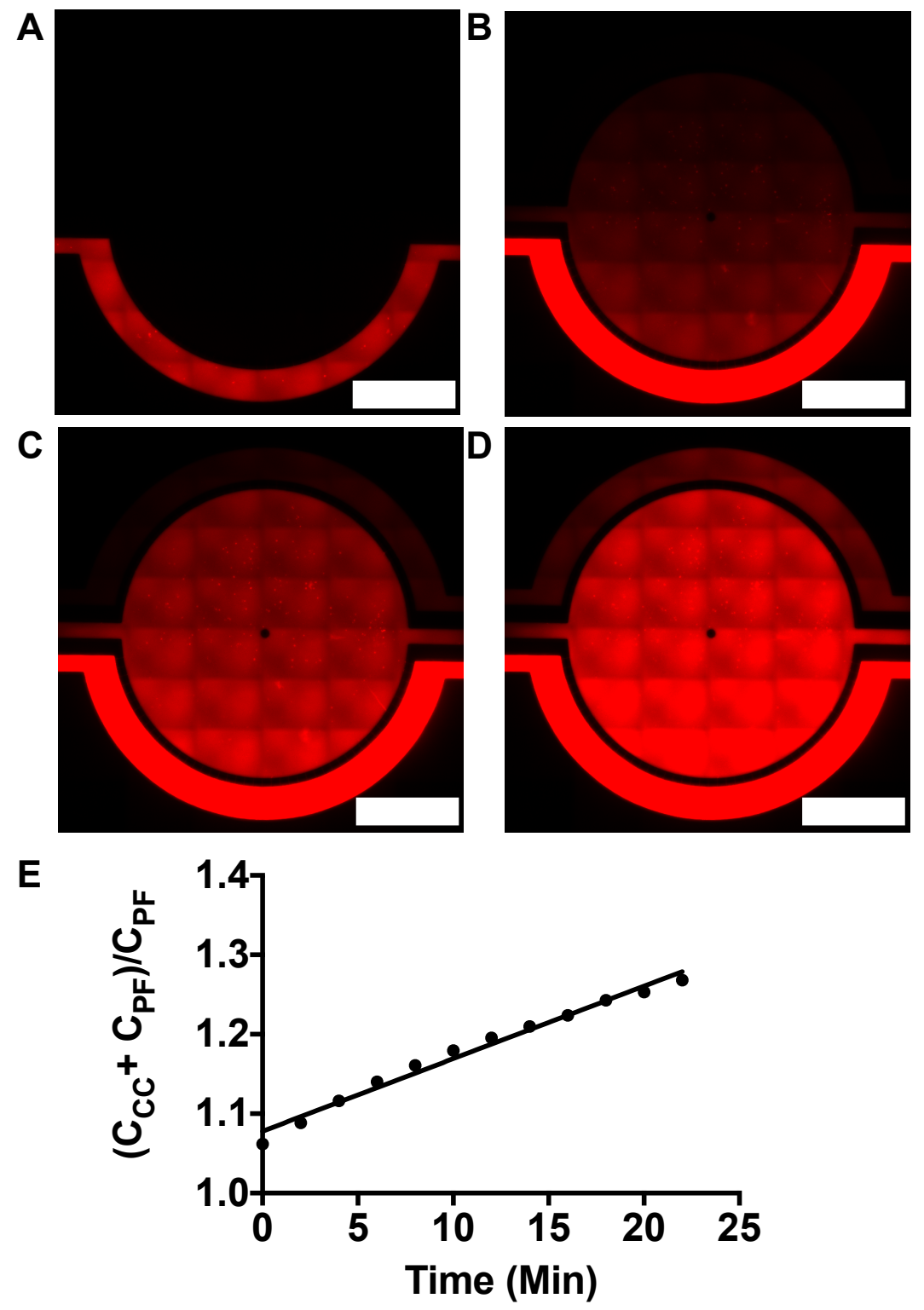

Figure 3.4: Representative time-lapse images showing passive diffusion of Free TRD from the outer to the central compartment.

Intensity of fluorescence increases linearly over time $0 \mathrm{~min}(\mathbf{A}), 30 \mathrm{~min}(\mathbf{B}), 60 \mathrm{~min}(\mathbf{C})$, and $90 \mathrm{~min}(\mathbf{D})$. (E) Linear concentration of tracer movement vs time to determine diffusion constants $\left(\mathrm{K}_{\mathrm{in}}\right)$. 
A

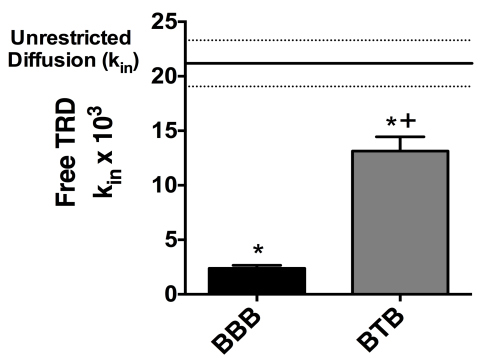

B

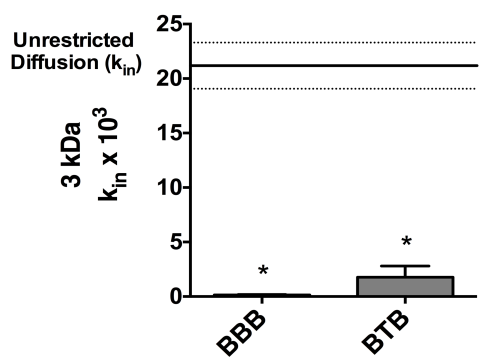

C

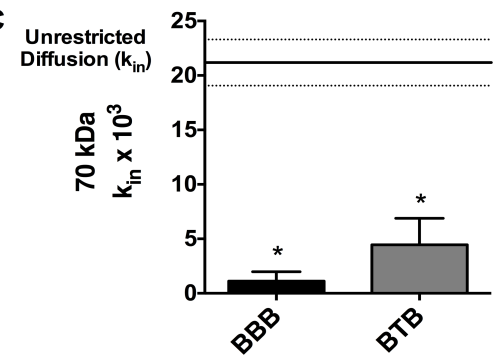

Figure 3.5: Linear central compartment accumulation of different tracers in the BBB and BTB microfluidic chip models.

Linear central compartment accumulation of Free TRD (A), TRD 3kDa (B), and TRD 70 $\mathrm{kDa}(\mathbf{C})$ in BBB and BTB SynVivo chip models. Images show rate of each tracer within each model. Statistical significance was determined using one-way ANOVA followed by Tukey's multiple comparison tests, and student's $t$-test; * $\mathrm{p}<0.05$ significance between tracer and unrestricted diffusion $\mathrm{k}_{\mathrm{in}}, \mathrm{n}=3-6 ;+\mathrm{p}<0.05$ significance between $\mathrm{BBB}$ and BTB models, $\mathrm{n}=3-6$. All data represent mean $\pm \mathrm{SEM}$. 

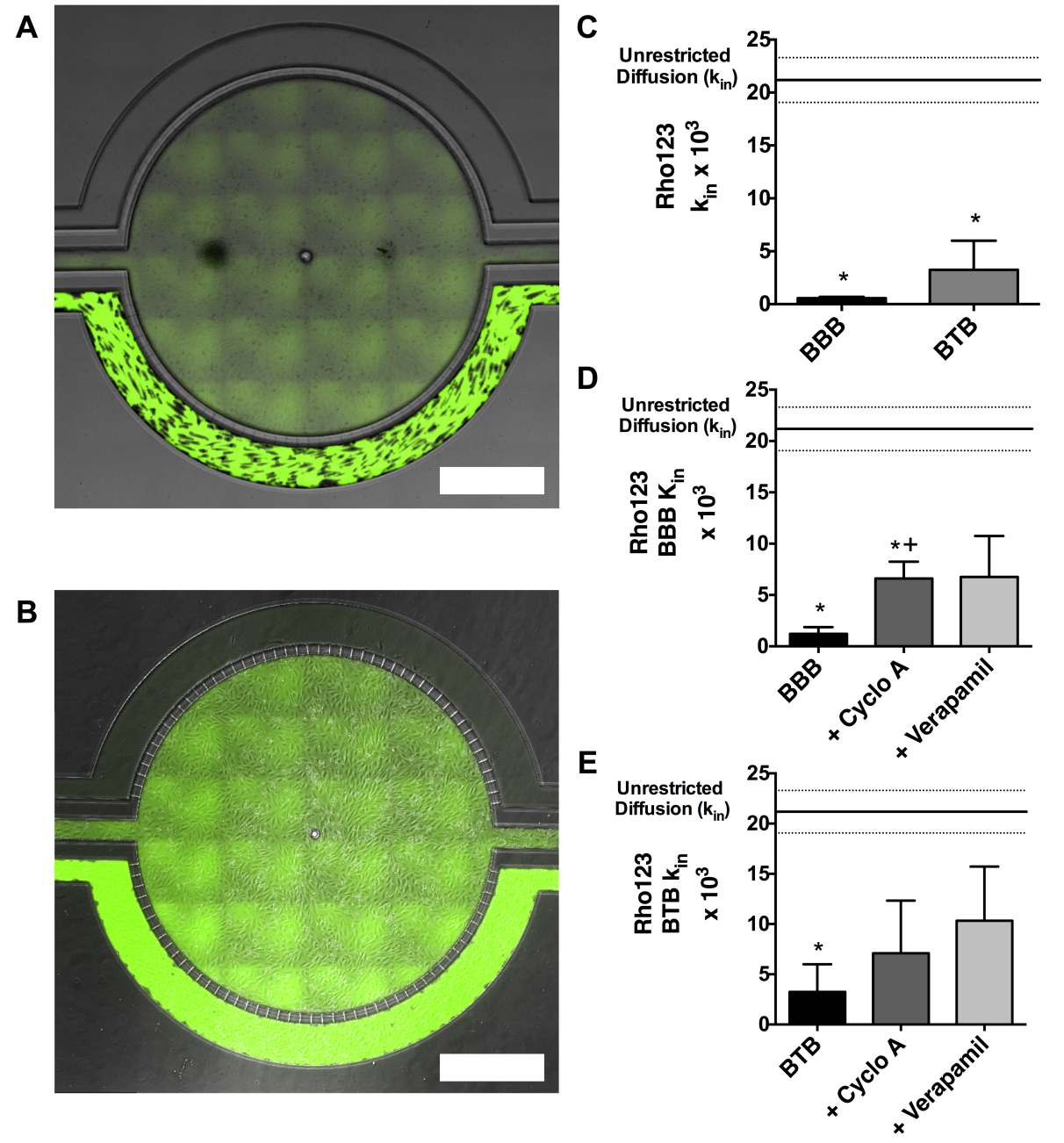

Figure 3.6: Rhodamine-123 permeability with and without inhibitors in BBB and BTB models.

Representative brightfield image of Rho123 dye accumulation in the central compartment after 90 minutes of perfusion in the BBB model without an inhibitor (A) and with an inhibitor (B). Rate of fluorescent dye accumulation of Rho123 into central compartment after 90 min of dye perfusion in BBB, and BTB chips (C). Rate of fluorescent dye accumulation in BBB (D) and BTB (E) chips perfused with Rho123 +/- p-gp inhibitors (cyclosporine A or verapamil). Statistical significance was determined using one-way 
ANOVA followed by Tukey's multiple comparison tests, and student's $t$-test; * $\mathrm{p}<0.05$ significance between tracer and unrestricted diffusion $\mathrm{k}_{\mathrm{in}}, \mathrm{n}=3-4 ;+\mathrm{p}<0.05$ significance between $\mathrm{BBB} / \mathrm{BTB}$ models and the addition of inhibitor, $n=3-6$. All data represent mean $\pm \mathrm{SEM}$ 


\subsection{References}

Abbott, N. J. (2002). "Astrocyte-endothelial interactions and blood-brain barrier permeability." J Anat 200(6): 629-638.

Abbott, N. J., A. A. Patabendige, D. E. Dolman, S. R. Yusof and D. J. Begley (2010). "Structure and function of the blood-brain barrier." Neurobiol Dis 37(1): 13-25.

Abbott, N. J., L. Ronnback and E. Hansson (2006). "Astrocyte-endothelial interactions at the blood-brain barrier." Nat Rev Neurosci 7(1): 41-53.

Adkins, C. E., R. K. Mittapalli, V. K. Manda, M. I. Nounou, A. S. Mohammad, T. B. Terrell, K. A. Bohn, C. Yasemin, T. R. Grothe, J. A. Lockman and P. R. Lockman (2013). "P-glycoprotein mediated efflux limits substrate and drug uptake in a preclinical brain metastases of breast cancer model." Front Pharmacol 4: 136.

Adkins, C. E., A. S. Mohammad, T. B. Terrell-Hall, E. L. Dolan, N. Shah, E. Sechrest, J. Griffith and P. R. Lockman (2016). "Characterization of passive permeability at the blood-tumor barrier in five preclinical models of brain metastases of breast cancer." Clin Exp Metastasis 33(4): 373-383.

Bakay, L., H. T. Ballantine, Jr., T. F. Hueter and D. Sosa (1956). "Ultrasonically produced changes in the blood-brain barrier." AMA Arch Neurol Psychiatry 76(5): $457-467$. 
Barry, P. H. and J. M. Diamond (1984). "Effects of unstirred layers on membrane phenomena." Physiol Rev 64(3): 763-872.

Bicker, J., G. Alves, A. Fortuna and A. Falcao (2014). "Blood-brain barrier models and their relevance for a successful development of CNS drug delivery systems: a review." Eur J Pharm Biopharm 87(3): 409-432.

Booth, R. and H. Kim (2012). "Characterization of a microfluidic in vitro model of the blood-brain barrier (muBBB)." Lab Chip 12(10): 1784-1792.

Brightman, M. W. and T. S. Reese (1969). "Junctions between intimately apposed cell membranes in the vertebrate brain." J Cell Biol 40(3): 648-677.

Coisne, C., L. Dehouck, C. Faveeuw, Y. Delplace, F. Miller, C. Landry, C. Morissette, L. Fenart, R. Cecchelli, P. Tremblay and B. Dehouck (2005). "Mouse syngenic in vitro blood-brain barrier model: a new tool to examine inflammatory events in cerebral endothelium." Lab Invest 85(6): 734-746.

Cordon-Cardo, C., J. P. O'Brien, J. Boccia, D. Casals, J. R. Bertino and M. R. Melamed (1990). "Expression of the multidrug resistance gene product (Pglycoprotein) in human normal and tumor tissues." J Histochem Cytochem 38(9): $1277-1287$. 
Crone, C. and O. Christensen (1981). "Electrical resistance of a capillary endothelium." J Gen Physiol 77(4): 349-371.

Crone, C. and S. P. Olesen (1982). "Electrical resistance of brain microvascular endothelium." Brain Res 241(1): 49-55.

Cucullo, L., M. Hossain, V. Puvenna, N. Marchi and D. Janigro (2011). "The role of shear stress in Blood-Brain Barrier endothelial physiology." BMC Neurosci 12: 40.

Cucullo, L., N. Marchi, M. Hossain and D. Janigro (2011). "A dynamic in vitro BBB model for the study of immune cell trafficking into the central nervous system." $\underline{J}$ Cereb Blood Flow Metab 31(2): 767-777.

Czupalla, C. J., S. Liebner and K. Devraj (2014). "In vitro models of the blood-brain barrier." Methods Mol Biol 1135: 415-437.

da Costa, J. C. (1972). "[Influence of electroconvulsions on the permeability of the blood-brain barrier to trypan blue]." Arq Neuropsiquiatr 30(1): 1-7.

Daneman, R. and A. Prat (2015). "The blood-brain barrier." Cold Spring Harb Perspect Biol 7(1): a020412. 
Demeule, M., D. Shedid, E. Beaulieu, R. F. Del Maestro, A. Moghrabi, P. B. Ghosn, R. Moumdjian, F. Berthelet and R. Beliveau (2001). "Expression of multidrugresistance P-glycoprotein (MDR1) in human brain tumors." Int J Cancer 93(1): 62-66.

Deo, A. K., F. P. Theil and J. M. Nicolas (2013). "Confounding parameters in preclinical assessment of blood-brain barrier permeation: an overview with emphasis on species differences and effect of disease states." Mol Pharm 10(5): 1581-1595.

Deosarkar, S. P., B. Prabhakarpandian, B. Wang, J. B. Sheffield, B. Krynska and M. F. Kiani (2015). "A Novel Dynamic Neonatal Blood-Brain Barrier on a Chip." PLoS One 10(11): e0142725.

Ehrlich, P. $\quad$ R. (1885). "Das sauerstufbudurfnis des organismus." Einefarbenanalytische Studie: 167.

Ferrara, N. and T. Davis-Smyth (1997). "The biology of vascular endothelial growth factor." Endocr Rev 18(1): 4-25.

Folkman, J. (1971). "Tumor angiogenesis: therapeutic implications." N Engl J Med 285(21): 1182-1186.

Garberg, P., M. Ball, N. Borg, R. Cecchelli, L. Fenart, R. D. Hurst, T. Lindmark, A. Mabondzo, J. E. Nilsson, T. J. Raub, D. Stanimirovic, T. Terasaki, J. O. Oberg and T. 
Osterberg (2005). "In vitro models for the blood-brain barrier." Toxicol In Vitro 19(3): 299-334.

Ghosh, A., D. O. Scott and T. S. Maurer (2014). "Towards a unified model of passive drug permeation I: origins of the unstirred water layer with applications to ionic permeation." Eur J Pharm Sci 52: 109-124.

Golden, P. L. and W. M. Pardridge (1999). "P-Glycoprotein on astrocyte foot processes of unfixed isolated human brain capillaries." Brain Res 819(1-2): 143-146.

Goldmann, E. ( 1913). "Vitalfarbung am zentralnervensystem. ." Abhandl Konigl preuss: Akad Wiss 1:1-60.

Griep, L. M., F. Wolbers, B. de Wagenaar, P. M. ter Braak, B. B. Weksler, I. A. Romero, P. O. Couraud, I. Vermes, A. D. van der Meer and A. van den Berg (2013). "BBB on chip: microfluidic platform to mechanically and biochemically modulate blood-brain barrier function." Biomed Microdevices 15(1): 145-150.

Hawkins, B. T. and T. P. Davis (2005). "The blood-brain barrier/neurovascular unit in health and disease." Pharmacol Rev 57(2): 173-185.

Hawkins, B. T. and R. D. Egleton (2006). "Fluorescence imaging of blood-brain barrier disruption." J Neurosci Methods 151(2): 262-267. 
Helms, H. C., N. J. Abbott, M. Burek, R. Cecchelli, P. O. Couraud, M. A. Deli, C. Forster, H. J. Galla, I. A. Romero, E. V. Shusta, M. J. Stebbins, E. Vandenhaute, B. Weksler and B. Brodin (2016). "In vitro models of the blood-brain barrier: An overview of commonly used brain endothelial cell culture models and guidelines for their use." J Cereb Blood Flow Metab 36(5): 862-890.

Henson, J. W., C. Cordon-Cardo and J. B. Posner (1992). "P-glycoprotein expression in brain tumors." J Neurooncol 14(1): 37-43.

Herland, A., A. D. van der Meer, E. A. FitzGerald, T. E. Park, J. J. Sleeboom and D. E. Ingber (2016). "Distinct Contributions of Astrocytes and Pericytes to Neuroinflammation Identified in a 3D Human Blood-Brain Barrier on a Chip." PLoS One 11(3): e0150360.

Hobbs, S. K., W. L. Monsky, F. Yuan, W. G. Roberts, L. Griffith, V. P. Torchilin and R. K. Jain (1998). "Regulation of transport pathways in tumor vessels: role of tumor type and microenvironment." Proc Natl Acad Sci U S A 95(8): 4607-4612.

Korjamo, T., A. T. Heikkinen and J. Monkkonen (2009). "Analysis of unstirred water layer in in vitro permeability experiments." J Pharm Sci 98(12): 4469-4479.

Korjamo, T., A. T. Heikkinen, P. Waltari and J. Monkkonen (2008). "The asymmetry of the unstirred water layer in permeability experiments." Pharm Res 25(7): 17141722. 
Liebner, S., A. Fischmann, G. Rascher, F. Duffner, E. H. Grote, H. Kalbacher and H. Wolburg (2000). "Claudin-1 and claudin-5 expression and tight junction morphology are altered in blood vessels of human glioblastoma multiforme." Acta Neuropathol 100(3): 323-331.

Lin, N. U., L. Amiri-Kordestani, D. Palmieri, D. J. Liewehr and P. S. Steeg (2013). "CNS metastases in breast cancer: old challenge, new frontiers." Clin Cancer Res 19(23): 6404-6418.

Lin, S. R. and M. Kormano (1977). "Cerebral circulation after cardiac arrest. Microangiographic and protein tracer studies." Stroke 8(2): 182-188.

Lockman, P. R., R. K. Mittapalli, K. S. Taskar, V. Rudraraju, B. Gril, K. A. Bohn, C. E. Adkins, A. Roberts, H. R. Thorsheim, J. A. Gaasch, S. Huang, D. Palmieri, P. S. Steeg and Q. R. Smith (2010). "Heterogeneous blood-tumor barrier permeability determines drug efficacy in experimental brain metastases of breast cancer." $\underline{\text { Clin }}$ Cancer Res 16(23): 5664-5678.

Loftsson, T. (2012). "Drug permeation through biomembranes: cyclodextrins and the unstirred water layer." Pharmazie 67(5): 363-370.

Loscher, W. and H. Potschka (2005). "Blood-brain barrier active efflux transporters: ATP-binding cassette gene family." NeuroRx 2(1): 86-98. 
Loscher, W. and H. Potschka (2005). "Role of drug efflux transporters in the brain for drug disposition and treatment of brain diseases." Prog Neurobiol 76(1): 22-76.

Man, S., E. E. Ubogu, K. A. Williams, B. Tucky, M. K. Callahan and R. M. Ransohoff (2008). "Human brain microvascular endothelial cells and umbilical vein endothelial cells differentially facilitate leukocyte recruitment and utilize chemokines for T cell migration." Clin Dev Immunol 2008: 384982.

Mittapalli, R. K., V. K. Manda, K. A. Bohn, C. E. Adkins and P. R. Lockman (2013). "Quantitative fluorescence microscopy provides high resolution imaging of passive diffusion and P-gp mediated efflux at the in vivo blood-brain barrier." J Neurosci Methods 219(1): 188-195.

Nakagawa, H., D. R. Groothuis, E. S. Owens, J. D. Fenstermacher, C. S. Patlak and R. G. Blasberg (1987). "Dexamethasone effects on [125I]albumin distribution in experimental RG-2 gliomas and adjacent brain." J Cereb Blood Flow Metab 7(6): $687-701$.

Nemeroff, C. B. and F. D. Crisley (1975). "Monosodium L-glutamate-induced convulsions: temporary alteration in blood-brain barrier permeability to plasma proteins." Environ Physiol Biochem 5(6): 389-395.

Neufeld, G., T. Cohen, S. Gengrinovitch and Z. Poltorak (1999). "Vascular endothelial growth factor (VEGF) and its receptors." FASEB J 13(1): 9-22. 
Neuhaus, W., R. Lauer, S. Oelzant, U. P. Fringeli, G. F. Ecker and C. R. Noe (2006). "A novel flow based hollow-fiber blood-brain barrier in vitro model with immortalised cell line PBMEC/C1-2." J Biotechnol 125(1): 127-141.

Olesen, S. P. and C. Crone (1983). "Electrical resistance of muscle capillary endothelium." Biophys J 42(1): 31-41.

Pardridge, W. M. (2005). "Molecular biology of the blood-brain barrier." Mol Biotechnol 30(1): 57-70.

Pardridge, W. M. (2007). "Blood-brain barrier delivery." Drug Discov Today 12(1-2): $54-61$.

Plate, K. H., A. Scholz and D. J. Dumont (2012). "Tumor angiogenesis and antiangiogenic therapy in malignant gliomas revisited." Acta Neuropathol 124(6): 763775.

Prabhakarpandian, B., M. C. Shen, J. B. Nichols, I. R. Mills, M. SidorykWegrzynowicz, M. Aschner and K. Pant (2013). "SyM-BBB: a microfluidic Blood Brain Barrier model." Lab Chip 13(6): 1093-1101.

Reese, T. S. and M. J. Karnovsky (1967). "Fine structural localization of a bloodbrain barrier to exogenous peroxidase." J Cell Biol 34(1): 207-217. 
Santaguida, S., D. Janigro, M. Hossain, E. Oby, E. Rapp and L. Cucullo (2006). "Side by side comparison between dynamic versus static models of blood-brain barrier in vitro: a permeability study." Brain Res 1109(1): 1-13.

Sawada, T., Y. Kato, N. Sakayori, Y. Takekawa and M. Kobayashi (1999). "Expression of the multidrug-resistance P-glycoprotein (Pgp, MDR-1) by endothelial cells of the neovasculature in central nervous system tumors." Brain Tumor Pathol 16(1): 23-27.

Schettler, T. and C. N. Shealy (1970). "Experimental selective alteration of bloodbrain barrier by x-irradiation." J Neurosurg 32(1): 89-94.

Schlageter, K. E., P. Molnar, G. D. Lapin and D. R. Groothuis (1999). "Microvessel organization and structure in experimental brain tumors: microvessel populations with distinctive structural and functional properties." Microvasc Res 58(3): 312-328.

Serlin, Y., I. Shelef, B. Knyazer and A. Friedman (2015). "Anatomy and physiology of the blood-brain barrier." Semin Cell Dev Biol 38: 2-6.

Shen, S. and W. Zhang (2010). "ABC transporters and drug efflux at the blood-brain barrier." Rev Neurosci 21(1): 29-53. 
Smith, Q. R. and Y. Takasato (1986). "Kinetics of amino acid transport at the bloodbrain barrier studied using an in situ brain perfusion technique." Ann N Y Acad Sci 481: 186-201.

Srinivasan, B., A. R. Kolli, M. B. Esch, H. E. Abaci, M. L. Shuler and J. J. Hickman (2015). "TEER measurement techniques for in vitro barrier model systems." J Lab Autom 20(2): 107-126.

Stanimirovic, D. B., M. Bani-Yaghoub, M. Perkins and A. S. Haqqani (2015). "Blood-brain barrier models: in vitro to in vivo translation in preclinical development of CNS-targeting biotherapeutics." Expert Opin Drug Discov 10(2): 141-155.

Takasato, Y., S. I. Rapoport and Q. R. Smith (1984). "An in situ brain perfusion technique to study cerebrovascular transport in the rat." Am J Physiol 247(3 Pt 2): H484-493.

Tews, D. S., A. Nissen, C. Kulgen and A. K. Gaumann (2000). "Drug resistanceassociated factors in primary and secondary glioblastomas and their precursor tumors." J Neurooncol 50(3): 227-237.

Zlokovic, B. V. (2008). "The blood-brain barrier in health and chronic neurodegenerative disorders." Neuron 57(2): 178-201. 


\title{
CHAPTER 4
}

\author{
TRASTUZUMAB EFFICACY IN AN IN VIVO AND IN VITRO \\ MODEL OF BRAIN METASTASES OF BREAST CANCER
}

\subsection{Introduction}

Brain metastases are a fatal neurological complication of breast cancer which have historically been a major cause of morbidity. Women with symptomatic central nervous system (CNS) metastases have a median survival of approximately 4 months (Colzani, Liljegren et al. 2011). Furthermore, less than 2\% of women survive two years post-diagnosis (Zimm, Wampler et al. 1981). The risk of developing brain metastasis has been reported to range from 10 to $16 \%$ among advanced-stage breast cancer patients, making it the second most common cause of metastatic brain tumor after lung cancer (10-25\%) (Palmieri, Smith et al. 2006, Park, Park et al. 2009, Steeg, Camphausen et al. 2011, Lin 2013, Yeh, Yu et al. 2015).

Among the many associated risk factors in the development of brain metastases from breast cancer, hormone receptor status is one of the most important (SanchezMunoz, Plata-Fernandez et al. 2013). Within the HER2-positive subset, hormone receptor status appears to further define the risk of CNS relapse. Patients with hormone receptornegative/HER2-positive tumors experience increased risk of the CNS as site of first relapse as compared to patients with hormone receptor-positive/HER2-positive tumors 
(Bendell, Domchek et al. 2003, Palmieri, Bronder et al. 2007, Leyland-Jones 2009, VazLuis, Ottesen et al. 2012). Up to 37\% of patients with HER2-positive breast cancer relapse due to intracranial metastases, despite control of the peripheral tumors (Clayton, Danson et al. 2004, Witzel, Oliveira-Ferrer et al. 2016). Palmieri et al. demonstrated that Her-2 overexpression increases the outgrowth of metastatic tumors cells in the brain in breast carcinoma cell lines (Palmieri, Bronder et al. 2007). A limiting factor in the treatment of brain metastases is the inability of chemotherapy to reach the desired tumor location. This is due, in large part, to the presence of a strictly controlled and regulated complex network known as the blood-brain barrier (BBB).

The BBB is a physical and functional barrier limiting passive diffusion of extrinsic agents into brain (Ballabh, Braun et al. 2004, Dauchy, Miller et al. 2009, Abbott, Patabendige et al. 2010, Cook and Freedman 2011). The BBB is mainly formed of endothelial cells, in addition to pericytes, astrocytes and neuronal cells that play an important role in the function of the BBB (Rip, Schenk et al. 2009). BBB endothelial cells have specific characteristics, such as the expression of tight junctions, which prevent passive paracellular transport of most water soluble compounds and many lipid soluble compounds with the exception of small gaseous compounds like carbon dioxide and molecular water (Ballabh, Braun et al. 2004, Hawkins and Davis 2005, Rip, Schenk et al. 2009, Abbott, Patabendige et al. 2010, Abbott and Friedman 2012, van Tellingen, YetkinArik et al. 2015). 
The function and organization of the BBB may be altered under pathological conditions. In the case of tumors, the BBB's structure and integrity are altered forming the "Blood-Tumor Barrier" (BTB) (van Tellingen, Yetkin-Arik et al. 2015). The BTB differs from the BBB in its decreased tight junction expression (Liebner, Fischmann et al. 2000), a disruption of the basement membrane (Deo, Theil et al. 2013) and an increase in permeability (Tate and Aghi 2009, Puhalla, Elmquist et al. 2015). However, radiologic data have shown that not all brain metastases display elevated BTB permeability (Lin, Bellon et al. 2004). The changes in BTB vascular permeability are typically heterogeneous throughout the tumor site (Lockman, Mittapalli et al. 2010, Villanueva 2013). It has been observed that brain metastases from HER2+ breast cancers infiltrate brain parenchyma without disrupting the $\mathrm{BBB}$, unlike brain metastases from triple negative or basal-type breast cancers, which often disrupt the BBB (Yonemori, Tsuta et al. 2010, Vaz-Luis, Ottesen et al. 2012, Witzel, Oliveira-Ferrer et al. 2016). Targeted therapies have revolutionized cancer treatment, potentially offering an improved therapeutic ratio (Boskovitz, Wikstrand et al. 2004), such as small molecule inhibitors (Hoelder, Clarke et al.) and monoclonal antibodies (Boskovitz, Wikstrand et al. 2004). However, the ability of these drugs and antibodies to permeate the brain and brain metastases is highly debated.

In this work, we have tested the permeability of $\mathrm{I}^{125}$-trastuzumab in an in vivo, and fluorescent trastuzumab-Rho123 (t-Rho123) in a novel in vitro model of brain metastases of breast cancer. This is one of the first studies to measure antibody movement across the 
blood-brain and blood-tumor barriers, demonstrating accumulation of trastuzumab in brain metastases of breast cancer with confirmatory experiments in vitro.

\subsection{Materials and Methods}

\subsubsection{In Vitro Studies:}

\subsubsection{Chemicals and Reagents}

Texas Red 70,000 MW Dextran (TRD $70 \mathrm{kDa}$ ) was purchased from Molecular Probes (Invitrogen, Carlsbad, CA). Trastuzumab (Herceptin ${ }^{\circledR}$, Genentech/ Roche) was buffer-exchanged into $50 \mathrm{mM}$ potassium phosphate buffer and $150 \mathrm{mM}$ sodium chloride adjusted to $\mathrm{pH}$ of 6.7. Trastuzumab was fluorescently linked to Rhodamine-123 (Innova Biosciences, Babraham, England). All other chemicals are of analytical grade and were purchased from Sigma-Aldrich (St. Louis, MO).

\subsubsection{Cell culture for in vitro studies}

Human Umbilical Vein Endothelial Cells (HUVECs) were were purchased from Lonza (Allendale, NJ). CTX-TDR2 rat brain astrocyte cell line were harvested, expanded, and generously provided by the laboratory of Dr. Jim Simpkins (West Virginia University, Morgantown, WV). Both HUVEC and astrocyte line were cultured and maintained in Endothelial Basal Medium - 2 (EBM-2) with the supplementation of EGM-2 BulletKits from Lonza (Allendale, NJ). The laboratory or Dr. Patricia Steeg, of the National Cancer Institute, generously provided a JIMT-1 brain metastases of breast cancer cell line, a line which naturally overexpresses HER2+. These cells were cultured and maintained in DMEM supplemented with $10 \%$ Fetal bovine serum and 1\% penstrep. 
All cell lines for in vitro studies were grown within a $37^{\circ} \mathrm{C}$ humidified incubator with $5 \%$ $\mathrm{CO}_{2}$ until $\sim 85-90 \%$ confluent.

\subsubsection{Cell Culture in Microfluidic Chip}

The co-culture idealized microvascular microfluidic chips used in this study were obtained from SynVivo Inc (Huntsville, AL). These microfluidic chips were prepared, then cultured with cells and maintained as previously described (SynVivo, Chapter 3) (Prabhakarpandian, Shen et al. 2013).

\subsubsection{Transport studies and quantification using fluorescent microscopy}

For each device, a BD Leur-lok syringe connected to Tygon tubing was filled with EBM2 media containing fluorescent tastuzumab and mounted on a programmable Harvard PHD 2000 syringe pump (Harvard Apparatus, Holliston, MA). Chips were maintained at $37^{\circ} \mathrm{C}$ with $5 \% \mathrm{CO}_{2}$ and mounted in an automated stage enclosure on a Nikon Eclipse TE2000-E Live Cell Sweptfield Confocal microscope (Melville, NY). Permeability was measured through the perfusion of fluorescently labeled trastuzumab through the outer chamber at $0.1 \mu \mathrm{L} / \mathrm{min}$. Brightfield ( $25 \mathrm{~ms}$ exposure) and TRITC (200 $\mathrm{ms}$ exposure) images were acquired every two minutes for 90 minutes with a Photometrics CoolSnap HQ2 Monochrome CCD Camera (Tucson, AZ) with a 20x/0.75 Plan Fluor Phase Contrast objective with a total field of $6 \mathrm{X} 8$, stitching images using brightfield with a $10 \%$ overlay. Following acquisition, NIS Elements Imaging Software was used to determine Regions of Interest (ROI) and data exported to Prism 6.0. A line of best fit was determined using linear regression (Prism 6.0), and the slope represents the relative rate 
of accumulation of fluorescence $\left(\mathrm{k}_{\text {in }}\right)$ in the central chamber (representing drug concentration found in normal brain) divided by the amount of fluorescence in the outer chamber (representing drug concentration found in the BBB/BTB vasculature). Unless otherwise noted, data are presented as mean \pm S.E.M.

\subsubsection{Kinetic analysis}

Unidirectional uptake transfer constants $\left(\mathrm{k}_{\text {in }}\right)$ were calculated using the following equation:

$$
\left(\mathrm{C}_{\mathrm{CC}}+\mathrm{C}_{\mathrm{PF}}\right) / \mathrm{C}_{\mathrm{PF}}=\mathrm{k}_{\text {in }}(\mathrm{t})+\mathrm{O}_{\mathrm{C}}
$$

Where $\mathrm{C}_{\mathrm{CC}}$ is the sum intensity of fluorophore in the region of interest in the central compartment ( $\mathrm{au}$ ) at the end of perfusion, $\mathrm{C}_{\mathrm{PF}}$ is the sum intensity of fluorophore (au) in the region of interest within the outer compartment, $\mathrm{t}$ is the perfusion time in minutes from the time the device reached steady state, and $\mathrm{O}_{\mathrm{C}}$ is the calculated intercept $(\mathrm{T}=0$ min; "outer compartment volume" (au)). Since the device took 22 minutes to reach steady state, $\mathrm{t}=0$ minutes is 22 minutes after start of the experiment, but 0 minutes from the start

of steady state. After the determination of a perfusion time where an adequate amount of fluorescent marker was allowed to pass into brain, while still remaining in the linear uptake zone, $\mathrm{k}_{\mathrm{in}}$ was determined (Takasato, Rapoport et al. 1984, Smith and Takasato 1986).

\subsubsection{Statistical Analysis}

Using linear regression with best-fit values, the slope of the line $\left(\mathrm{k}_{\text {in }}\right)$ was determined. One-way ANOVA analysis, unpaired student $\mathrm{t}$ test's with Welch's correction, and an F 
test to compare variances were used for the comparison of kin values between the unrestricted diffusions, BBB, and BTB models,. For all data, errors are reported as standard error of the mean unless otherwise indicated. Differences were considered statistically significant at $\mathrm{p}<0.05$. (GraphPad Prism version 6.00 for Mac, GraphPad Software, San Diego, CA, USA).

\subsubsection{In Vivo Studies:}

\subsubsection{Chemicals and reagents}

Texas red conjugated $625 \mathrm{MW}$ dextran (TRD $625 \mathrm{Da}$ ) was purchased from Molecular Probes (Invitrogen, Carlsbad, CA). Trastuzumab (Roche) was buffer-exchanged into 50 $\mathrm{mM}$ potassium phosphate buffer and $150 \mathrm{mM}$ sodium chloride adjusted to $\mathrm{pH}$ of 6.7 . Trastuzumab was radiolabeled with ${ }^{125}$ I. All other chemicals are of analytical grade and were purchased from Sigma-Aldrich (St. Louis, MO).

\subsubsection{Cell culture}

Human MDA-MB-231-HER2+ metastatic breast cancer cells expressing enhanced green fluorescent protein (eGFP) and the luciferase construct, were cultured in DMEM supplemented with $10 \%$ fetal bovine serum and zeocin $(300 \mu \mathrm{g} / \mathrm{ml})$. Cells were harvested at $80 \%$ confluency for intracardiac injection. All cell lines were generously provided by the laboratory of Dr. Patricia Steeg at the National Cancer Institute. 


\subsubsection{Experimental brain metastases model}

Homozygous Female $\mathrm{NuNu}(\mathrm{n}=20)$ mice were obtained from Charles River Laboratories

(Kingston, NY) and used for all experiments in this study. All animals were 6-8 weeks of age at the initiation of the metastases models and were housed in a barrier facility. All studies were approved by the Animal Care and Use Committee at Texas Tech University Health Sciences Center and conducted in accordance with the 1996 NIH Guide for the Care and Use of Laboratory Animals. Mice were anesthetized with 2\% isoflurane and inoculated with 175,000 breast cancer cells in the left cardiac ventricle with the aid of a stereotaxic device (Stoelting, Wood Dale, IL). The inoculum circulates in the peripheral vasculature, arrests in brain capillaries, extravasates across the blood-brain barrier (BBB), and mice develop metastatic lesions predominantly in the brain (Lockman, Mittapalli et al. 2010). After intracardiac injection, mice were placed in a warmed $\left(37^{\circ} \mathrm{C}\right)$ sterile cage and their vitals monitored until fully recovered. Metastases were allowed to develop and visualized with bioluminescent imaging, until neurologic symptoms appeared ( $\sim 32$ days), and animals were anesthetized with ketamine/xylazine (100 and $8 \mathrm{mg} / \mathrm{kg}$ respectively) prior to injection with ${ }^{125}$ I-trastuzumab via IV bolus dose (femoral vein). ${ }^{125}$ I-trastuzumab was allowed to circulate for 24h. TRD $625 \mathrm{Da}$ was injected intravenously (femoral vein). 10 minutes' post-injection, blood samples were obtained and mice were euthanized decapitated.

\subsubsection{Harvesting of the brain and other tissues and organs}

Animals were euthanized, brain tissue rapidly removed (less than 60 seconds), and placed in isopentane $\left(-65^{\circ} \mathrm{C}\right)$. Brains were sliced $(20 \mu \mathrm{m})$ using a cryostat (Leica Microsystems, 
Wetzler, Germany), and sections were mounted on charged gold plated glass slides, air dried, and stored at $-80^{\circ} \mathrm{C}$. In addition to the brain, blood and samples from other organs (heart, lungs, liver, spleen, kidney) were collected, washed, and weighed for comparative analysis. Radioactivity was measured immediately following collection (Tri-CARB 2900TR, Perkin Elmer) and expressed as cpm/mg then converted to nCi/g. Distribution ratios are expressed as the amount of radioactivity in the tissue/blood normalized by weight.

\subsubsection{Quantitative autoradiography (QAR)}

Slides were placed in QAR cassettes (FujiFilm Life Sciences, Stamford, CT) along with ${ }^{125}$ I autoradiographic standards (Amersham Biosciences). A phosphor screen (FujiFilm Life Sciences, $20 \times 40$ super-resolution) was placed on the slides and standards and allowed to develop for up to 14 days. QAR phosphor screens were developed in a high-resolution phosphor-imager (FUJI FLA-7000, FujiFilm Life Sciences) and

converted to digital images. Digital QAR images were calibrated to ${ }^{125}$ I standards and analyzed using MCID Analysis software (InterFocus Imaging LTD, Linton, Cambridge, England). Metastases permeability fold-changes were calculated based on ${ }^{125}$ I signal intensity within confirmed metastases locations (determined by eGFP fluorescence image overlays) relative to ${ }^{125}$ I signal intensity in normal brain. 


\subsubsection{Fluorescence measurement}

Texas red fluorescence was imaged using a DsRed sputter filter (excitation/band $\lambda$ $545 / 25 \mathrm{~nm}$, emission/band $\lambda 605 / 70 \mathrm{~nm}$ and dichromatic mirror at $\lambda 565 \mathrm{~nm})($ Chroma Technologies, Bellows Falls, VT) and eGFP (expressed in MDA-MB-231BR-HER2+) using an ET-GFP sputter filter (excitation/band $\lambda 470 / 40 \mathrm{~nm}$, emission/band $\lambda$ 525/50 nm and dichromatic mirror at $\lambda 495 \mathrm{~nm})($ Chroma Technologies, Bellows Falls, VT). Fluorescence image capture and analysis software (SlideBook 5.0; Intelligent Imaging Innovations Inc., Denver, CO) was used to obtain and quantify fluorescence images. Texas red permeability fold-changes were determined by Texas Red Sum intensity (SI) per unit area of metastases relative to the SI per area of contralateral normal brain regions. If metastases occurred in contralateral regions, adjacent slices containing unaffected tissues of the same brain structure were used as comparative normal brain regions.

\subsubsection{Unidirectional uptake transfer constants $\left(K_{\text {in }}\right)$}

$\mathrm{K}_{\text {in }}$ values were then calculated from brain distribution volume versus time as previously described (Lockman, Mittapalli et al. 2010).

\subsubsection{Bioluminescent imaging}

Mice were injected with D-luciferin potassium salt (150mg/kg; PerkinElmer, Waltham, MA) dissolved in sterile 1X PBS via intraperitoneal (IP) injection and then anesthetized with $2 \%$ isoflurane. Fifteen minutes after IP injection of D-luciferin, darkfield images of mice were acquired with an IVIS Lumineer XV (PerkinElmer) to 
detect bioluminescence. Animals were imaged 1, 3, 6, 9, 12, 24, 48, 72, 96, 120, 144, and 168 hours post intracardiac injection to ensure successful tumor injection and growth.

\subsubsection{Data analysis}

Statistical significance was determined by Student's t-test and one-way ANOVA followed by Bonferroni's multiple comparison's tests. All differences were considered statistically significant at $p<0.05$. Data is reported as Mean \pm Standard Deviation (SD) unless otherwise noted (GraphPad Prism 7.0, San Diego, CA). Results associated with drug concentration in tumor and brain distant to tumor (BDT) are Mean values of combined readings from all tumor and BDT areas in the study group without separation by individual animal data. In the case of $\mathrm{K}_{\text {in }}$ analysis (Lockman, Mittapalli et al. 2010), values obtained at individual time points were also pooled together.

\subsection{Results}

To visualize in vitro movement of t-Rho123, microfluidic BBB and BTB chips (Fig. 4.1A) were established and utilized as previously published (Prabhakarpandian, Shen et al. 2013). The distribution of t-Rho123 in BBB and BTB models was analyzed. Using Equation 4.1, we observed a linear increase of fluorescent trastuzumab uptake in both the BBB $\left(0.27 \pm 0.33 \times 10^{4}\right)$ (Fig. 4.1C) and BTB $\left(1.29 \pm 0.93 \times 10^{4}\right)$ (Fig. 4.1D) models significantly greater than $0(\mathrm{p}<0.05)$. The rate of movement of fluorescent trastuzumab was quantified through the addition of a region of interest in the outer chamber (comparative to concentration of drug in plasma, $\mathrm{C}_{\mathrm{PF}}$ ) and a region of interest in the central chamber (comparative to concentration of drug in brain, $\mathrm{C}_{\mathrm{CC}}$ ), then divided by 
the sum intensity of tracer in the outer chamber $\left(\mathrm{C}_{\mathrm{CC}}+\mathrm{C}_{\mathrm{PF}} / \mathrm{C}_{\mathrm{PF}}\right)$ and plotted over time. The slope of this line, Kin $\left(\mu \mathrm{L} / \mathrm{min} / \mu \mathrm{m}^{2}\right)$, was plotted and graphed (Fig. 4.1B) as the mean \pm S.E.M. for the BBB $(0.18 \pm 0.05, n=3)$ and BTB $(2.12 \pm 1.36, n=3)$ models. Both the Kin for the BBB models as well as the heterogeneity of the Kin values in the BTB models were comparable to in vivo. The BBB $(\mathrm{p}<0.0033)$ and BTB $(\mathrm{p}<0.0005)$ models were significantly different in comparison to the unrestricted diffusion $\mathrm{K}_{\text {in }}$ of this model, as previously described (SynVivo, Chapter 3).

Organ distribution of trastuzumab after intracardiac (left ventricle) injection of 20 $\mathrm{Nu} / \mathrm{Nu}$ mice with the HER2+ breast cancer cell line was determined. After the mice were developed metastases ( $\sim 32$ days), radio-labeled ${ }^{125}$ I-trastuzumab was injected and allowed to circulate, followed by the administration of TRD 10 minutes prior to decapitation. Quantitative autoradiography (QAR) was used to measure the brain tissue distribution of ${ }^{125}$ I-trastuzumab. Figure 4.2A represents organ distribution of ${ }^{125} \mathrm{I}$ trastuzumab, variability in different body organs is observed. ${ }^{125}$ I-trastuzumab was found in significant quantities in spleen $(5.04 \%, \mathrm{SD}=3.91)$, lungs $(4.45 \%, \mathrm{SD}=2.08)$, liver $(3.54 \%, \mathrm{SD}=2.26)$, kidney $(3.12 \%, \mathrm{SD}=2.06)$, and heart $(3.08 \%, \mathrm{SD}=1.78)$ compared to normal brain $(0.30 \%, \mathrm{SD}=0.22)$ and tumor brain tissues $(0.46 \%, \mathrm{SD}=0.46)$. The accumulation of ${ }^{125}$ I-trastuzumab in tumor brain was 1.5 fold higher than normal brain tissue $(\mathrm{p}<0.0001)$ (Fig. 4.2B).

Heterogeneous and limited distribution of ${ }^{125}$ I-trastuzumab in a preclinical brain metastases of breast cancer model is shown in Figure 4.3A- 4.3C. Metastases were 
categorized into four groups based upon the magnitude of permeability change compared to normal brain; where low, intermediate, medium and high corresponds to $<$ mean brain $+3 \mathrm{xSD} ;>$ mean brain $+3 \mathrm{xSD}$ but $<2$ fold; $2-4$ fold; high is $>4$ fold, respectively. The mean and standard deviation of the four groups were, 1.30 and 0.34 for low permeability, 1.88 and 0.07 for intermediate permeability, 2.79 and 0.61 for medium permeability, and 7.40 and 4.66 for high permeability (Fig. 4.3D).

Fold increase in ${ }^{125}$ I-trastuzumab (over normal brain) was plotted versus metastasis size $\left(\mathrm{mm}^{2}\right)$ in individual 231-Br-Her2 brain metastases (Fig. 4.3E). No clear correlation was found between the size of brain metastases and the amount of ${ }^{125} \mathrm{I}$ trastuzumab localized (Fig. 4.3E). $\mathrm{K}_{\text {in }}$ values were determined separately for normal and tumor areas of the brain (Fig. 4.3F). Mean $\mathrm{K}_{\text {in }}$ for normal brain tissue was $1.457 \times 10^{7}$ $\mathrm{mL} / \mathrm{sec} / \mathrm{g}(\mathrm{SD}=0.55)$ while mean $\mathrm{K}_{\text {in }}$ in the case of tumor brain was $3.80 \mathrm{~mL} / \mathrm{sec} / \mathrm{g}(\mathrm{SD}=$ 2.17). There was minimal correlation between fold increase of ${ }^{125}$ I-trastuzumab and the lesion size.

\subsection{Discussion}

Treatment of brain metastases of breast cancer conventionally consists of surgery, whole brain radiation, stereotactic radiosurgery, chemotherapy, and/or biological therapies (Rostami, Mittal et al. 2016). Various chemotherapeutic agents have shown only a modest effect on survival due to their limited ability to cross the blood brain barrier (BBB) (Rostami, Mittal et al. 2016). In a preclinical study using two different models of brain metastases of breast cancer, most metastases exhibited some increased 
BTB permeability in comparison to normal brain. However, BTB permeability remained poorly correlated with lesion size, and only approximately $10 \%$ of lesions with the highest permeability exhibited cytotoxic responses to paclitaxel or doxorubicin (Lockman, Mittapalli et al. 2010). In low-grade gliomas, the BTB resembles a normal functioning BBB, while in high-grade gliomas, BTB is disrupted "leaky", as it is characterized by major alterations of the normal vascular function, shown through contrast-enhanced MRI by Dhermain et al. (Dhermain, Hau et al. , van Tellingen, YetkinArik et al. 2015). However, the magnitude of this local disruption is unlikely to be sufficient to allow drug penetration in meaningful quantities, and is thus considered a major obstacle for drug delivery to the brain (Tzeng and Green 2013).

Trastuzumab (Herceptin ${ }^{\circledR}$, Genentech/ Roche), is a widely used humanized monoclonal antibody, for the treatment of HER2+ breast cancer due to its ability to recognize and bind to the extracellular juxtamembrane domain of HER2. Through this binding, trastuzumab is able to inhibit the proliferation, therefore survival, of HER2dependent tumors (Park, Park et al. 2009). The ability of trastuzumab to significantly cross the BBB is unclear (Kute, Lack et al. 2004). Şendur et al (Sendur, Uncu et al. 2014) reported a case study using a combination of lapatinib and capecitabine followed by trastuzumab in HER2-positive brain metastatic breast cancer. No progression of cranial metastases was found post-treatment. In another case series by Mutlu et al (Mutlu and Buyukcelik 2015), one in three patients with HER-2 positive breast cancer brain metastasis maintained the brain metastases post-treatment with a combination of weekly trastuzumab plus vinorelbine. In an in vivo study by Kodack et al, it was observed that 
through the use of a combination of a HER2 inhibitor with an anti-VEGF receptor-2 antibody, trastuzumab, and lapatinib, tumor growth was significantly slowed in the brain, resulting in increased survival in a mouse model of HER2-amplified breast cancer brain metastasis using an orthotopic xenograft of BT474 cells (Kodack, Chung et al. 2012).

In patients without brain metastases, the ratio of trastuzumab in plasma to trastuzumab in cerebrospinal fluid is $>300: 1$ (Pestalozzi and Brignoli 2000). Lampson found that monoclonal antibodies were able to reach brain metastases in only 2 models (Lampson 2011): blood-borne tumor from outside the brain, and dormant tumor that grows enough to rupture the $\mathrm{BBB}$, and thus allow $\mathrm{mABs}$ to infiltrate. In addition to physical barriers, several functional barriers contribute to the restrictive nature of $\mathrm{BBB}$, which represents a major obstacle to effective drug delivery into the CNS (Deeken and Loscher 2007). A group of efflux transporters (such as P-glycoprotein, breast cancer resistance protein, and multidrug resistance-associated proteins) are expressed on the brain endothelia, and collectively cause rapid efflux of a large group of lipophilic drugs from the CNS (Loscher and Potschka 2005). The use of novel P-glycoprotein inhibitors in enhancing BBB permeation, drug uptake, and retention has been an area of recent investigation (Andersson, Hansen et al. 2013, Andersson, Badisco et al. 2014, Bauer, Karch et al. 2015).

This model has been previously used to observe small molecule movement and Pglycoprotein efflux (SynVivo, Chapter 3). Through the utilization of this device, we observed a relatively similar fold increase of trastuzumab in vivo as compared to the in 
vitro observation in the microfluidic device when comparing fold increases from the blood-brain barrier to the blood-tumor barrier. The prediction and evaluation of the ability of various therapeutic and diagnostic moieties to cross the BBB and BTB, as well as brain uptake kinetics are critical to progress efficient brain metastases therapy and diagnosis from basic to translational research. Such knowledge is urgently needed for the early detection and management of high-risk brain metastases in patients. This study demonstrates that, though in minute quantities, trastuzumab does in fact cross the bloodbrain and blood-tumor barriers. The heterogeneity of antibody dispersion observed within the in vivo tumors mimics that seen in the in vitro tumor model. Expanding on these data, future work should include the use of additional antibodies used to treat brain metastases of breast cancer, as well as the addition of different small molecule inhibitors. 

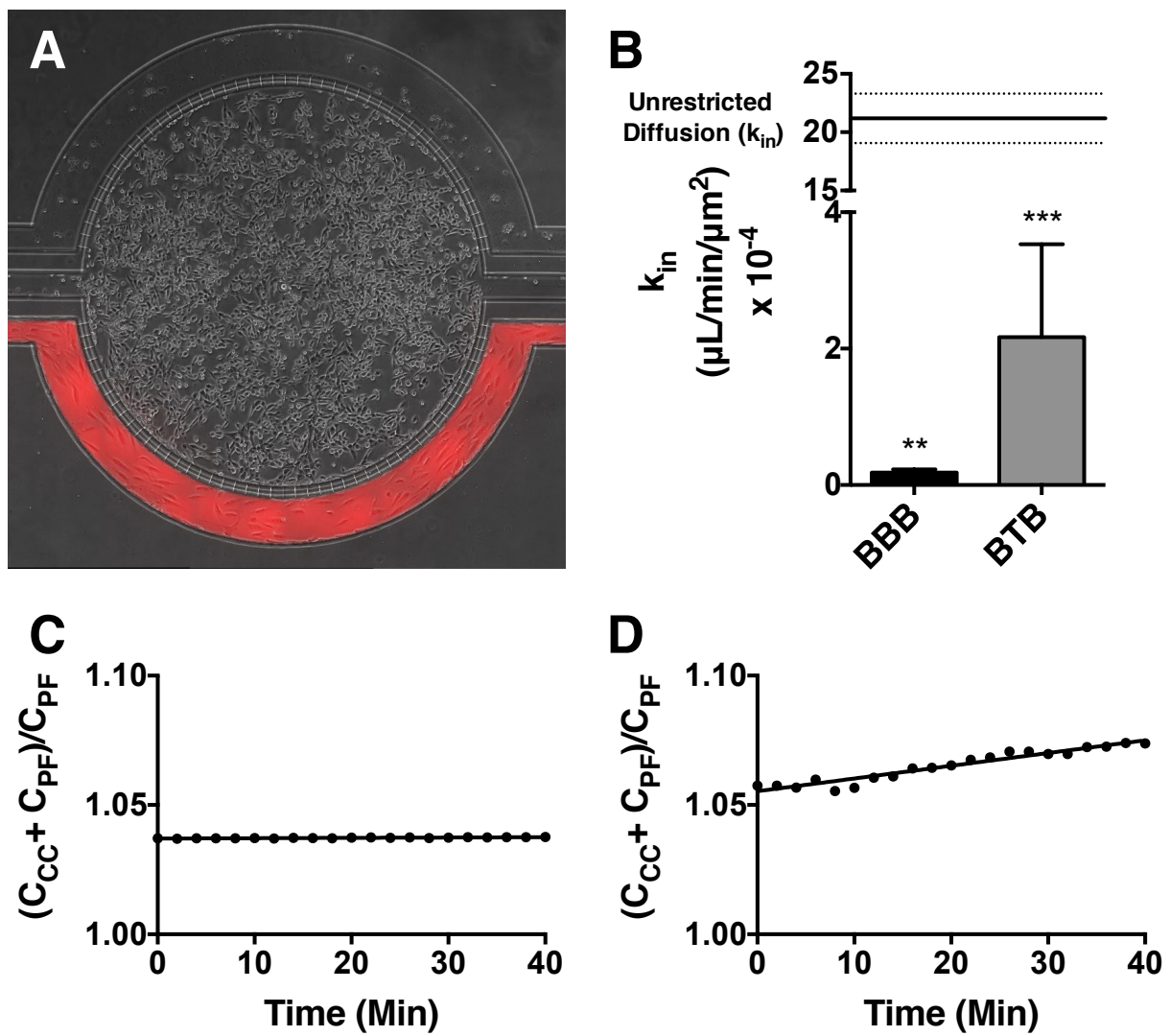

Figure 4.1: Mechanism of trastuzumab movement.

Linear central compartment accumulation of ${ }^{125}$ I-trastuzumab in in vitro BBB and BTB microfluidic chip models. Representative image of model with TRITC labeled ${ }^{125} \mathrm{I}-$ trastuzumab flowing over HUVEC cells in the outer compartment and either astrocytes or JIMT-1 cancer cells in the central compartment $(\mathbf{1 A})$. Rate of ${ }^{125}$ I-trastuzumab movement in each model plotted against the unrestricted diffusion $\mathrm{k}_{\mathrm{in}} ; * * \mathrm{p}<0.0033$ significance between $\mathrm{BBB}$ model and unrestricted diffusion $\mathrm{k}_{\mathrm{in}}, \mathrm{n}=3 ; * * * \mathrm{p}<0.0005$ significance between BTB model and unrestricted diffusion $\mathrm{k}_{\mathrm{in}}, \mathrm{n}=3$. All data represent mean \pm S.E.M. Each model is significantly different than $0(\mathrm{p}<0.05)(\mathbf{1 B})$. Representative graphs of the rate of accumulation of ${ }^{125} \mathrm{I}$ in the BBB (1C) and BTB (1D) microfluidic devices. 

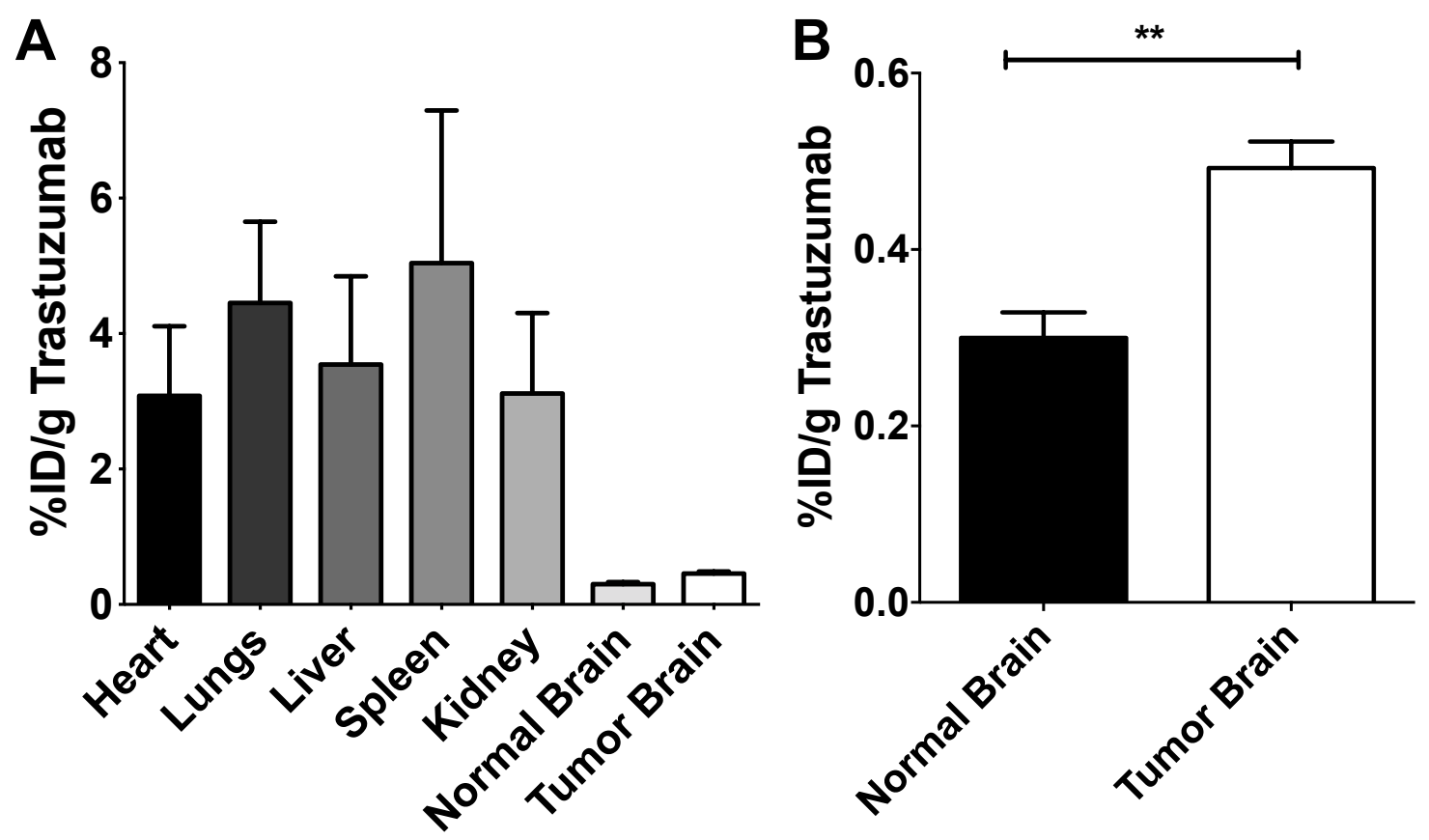

Figure 4.2. The distribution of radiolabeled ${ }^{125} \mathrm{I}$-trastuzumab in various body organs (a) and in normal and tumor brain tissues (b). 

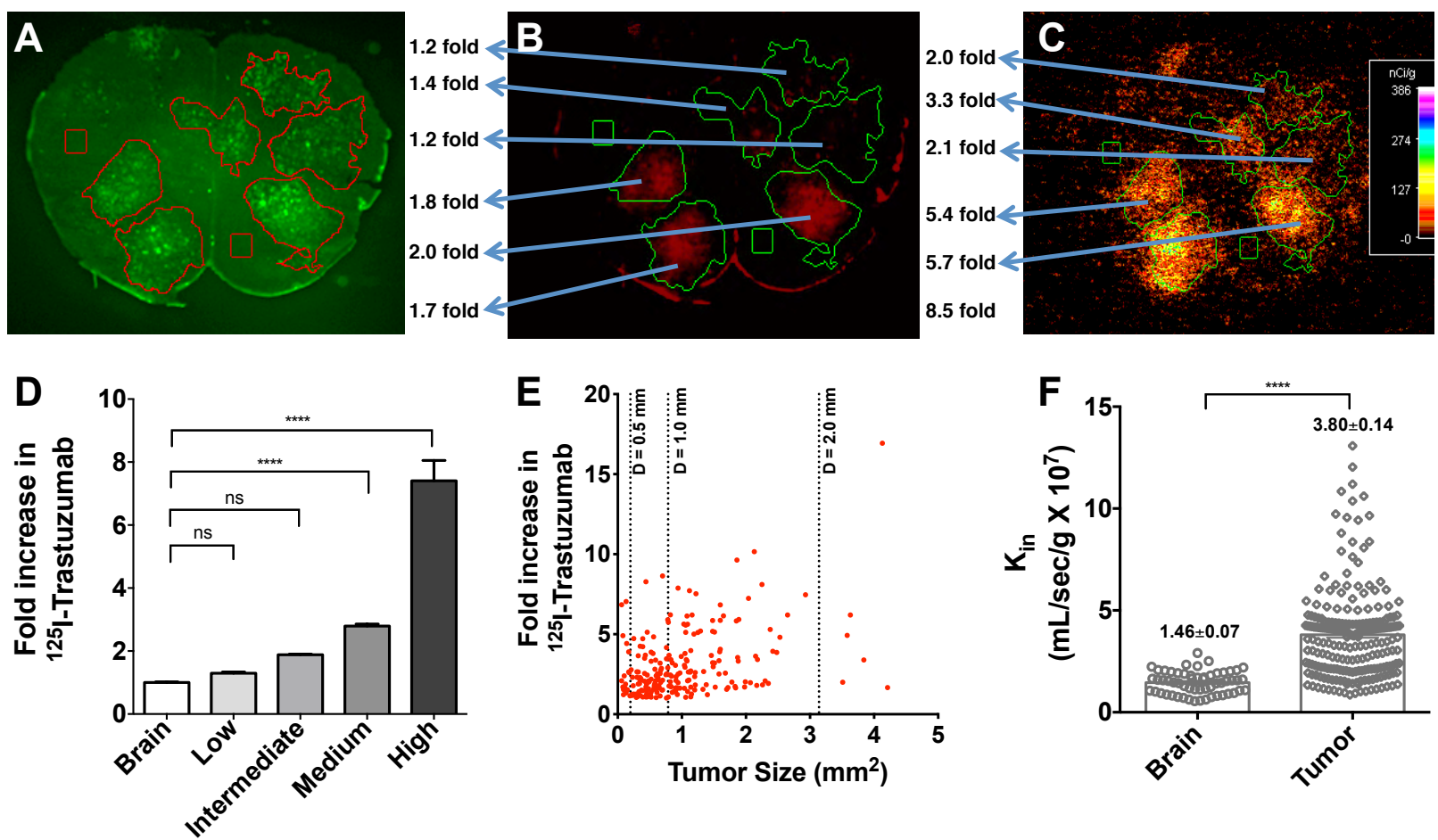

Figure 4.3. Heterogeneous and limited distribution of ${ }^{125}$ I-trastuzumab in preclinical brain metastases of breast cancer model.

Representative images of location of eGFP labeled 231-Br-Her2 brain metastases (3A), brain accumulation of Texas Red $625 \mathrm{Da}(\mathbf{3 B})$ and ${ }^{125} \mathrm{I}-$ trastuzumab (3C) are shown, respectively. Metastases were categorized into four groups based upon the magnitude of permeability change compared to normal brain; where low, intermediate and high corresponds to less than mean brain $+3 x$ SD; more than mean brain $+3 x$ SD but $<2$ fold; $2-4$ fold; and high is $>4$ fold, respectively (Values represent mean $\pm \mathrm{SD}, \mathrm{n}=251$ metastases) (3D). Fold increase in ${ }^{125}$ I-trastuzumab (over normal brain) was plotted versus metastasis size $\left(\mathrm{mm}^{2}\right)$ in individual $231-\mathrm{Br}-\mathrm{Her} 2$ brain metastases 
(3E). The correlation was minimal with ${ }^{125}$ I-trastuzumab fold increase versus lesion size. $\mathrm{K}_{\text {in }}$ values for normal and tumor areas of the brain $(\mathbf{3 F})$. 


\subsection{References}

Abbott, N. J. and A. Friedman (2012). "Overview and introduction: The blood-brain barrier in health and disease." Epilepsia 53(0 6): 1-6.

Abbott, N. J., A. A. Patabendige, D. E. Dolman, S. R. Yusof and D. J. Begley (2010). "Structure and function of the blood-brain barrier." Neurobiol Dis 37(1): 13-25.

Andersson, O., L. Badisco, A. H. Hansen, S. H. Hansen, K. Hellman, P. A. Nielsen, L. R. Olsen, R. Verdonck, N. J. Abbott, J. Vanden Broeck and G. Andersson (2014). "Characterization of a novel brain barrier ex vivo insect-based P-glycoprotein screening model." Pharmacol Res Perspect 2(4): e00050.

Andersson, O., S. H. Hansen, K. Hellman, L. R. Olsen, G. Andersson, L. Badolo, N. Svenstrup and P. A. Nielsen (2013). "The grasshopper: a novel model for assessing vertebrate brain uptake." J Pharmacol Exp Ther 346(2): 211-218.

Ballabh, P., A. Braun and M. Nedergaard (2004). "The blood-brain barrier: an overview: structure, regulation, and clinical implications." Neurobiol Dis 16(1): 1-13.

Bauer, M., R. Karch, M. Zeitlinger, C. Philippe, K. Romermann, J. Stanek, A. MaierSalamon, W. Wadsak, W. Jager, M. Hacker, M. Muller and O. Langer (2015). "Approaching complete inhibition of P-glycoprotein at the human blood-brain barrier: an (R)-[11C]verapamil PET study." J Cereb Blood Flow Metab 35(5): 743-746. 
Bendell, J. C., S. M. Domchek, H. J. Burstein, L. Harris, J. Younger, I. Kuter, C. Bunnell, M. Rue, R. Gelman and E. Winer (2003). "Central nervous system metastases in women who receive trastuzumab-based therapy for metastatic breast carcinoma." Cancer 97(12): 2972-2977.

Boskovitz, A., C. J. Wikstrand, C. T. Kuan, M. R. Zalutsky, D. A. Reardon and D. D. Bigner (2004). "Monoclonal antibodies for brain tumour treatment." Expert Opin Biol Ther 4(9): 1453-1471.

Clayton, A. J., S. Danson, S. Jolly, W. D. J. Ryder, P. A. Burt, A. L. Stewart, P. M. Wilkinson, R. S. Welch, B. Magee, G. Wilson, A. Howell and A. M. Wardley (2004). "Incidence of cerebral metastases in patients treated with trastuzumab for metastatic breast cancer." British Journal of Cancer 91(4): 639-643.

Colzani, E., A. Liljegren, A. L. Johansson, J. Adolfsson, H. Hellborg, P. F. Hall and K. Czene (2011). "Prognosis of patients with breast cancer: causes of death and effects of time since diagnosis, age, and tumor characteristics." J Clin Oncol 29(30): 4014-4021.

Cook, L. J. and J. Freedman (2011). Brain Tumors, Rosen Pub.

Dauchy, S., F. Miller, P. O. Couraud, R. J. Weaver, B. Weksler, I. A. Romero, J. M. Scherrmann, I. De Waziers and X. Decleves (2009). "Expression and transcriptional 
regulation of $\mathrm{ABC}$ transporters and cytochromes $\mathrm{P} 450$ in hCMEC/D3 human cerebral microvascular endothelial cells." Biochem Pharmacol 77(5): 897-909.

Deeken, J. F. and W. Loscher (2007). "The blood-brain barrier and cancer: transporters, treatment, and Trojan horses." Clin Cancer Res 13(6): 1663-1674.

Deo, A. K., F. P. Theil and J. M. Nicolas (2013). "Confounding parameters in preclinical assessment of blood-brain barrier permeation: an overview with emphasis on species differences and effect of disease states." Mol Pharm 10(5): 1581-1595.

Dhermain, F. G., P. Hau, H. Lanfermann, A. H. Jacobs and M. J. van den Bent "Advanced MRI and PET imaging for assessment of treatment response in patients with gliomas." Lancet Neurol 9(9): 906-920.

Hawkins, B. T. and T. P. Davis (2005). "The blood-brain barrier/neurovascular unit in health and disease." Pharmacol Rev 57(2): 173-185.

Hoelder, S., P. A. Clarke and P. Workman "Discovery of small molecule cancer drugs: Successes, challenges and opportunities." Molecular Oncology 6(2): 155-176.

Kodack, D. P., E. Chung, H. Yamashita, J. Incio, A. M. Duyverman, Y. Song, C. T. Farrar, Y. Huang, E. Ager, W. Kamoun, S. Goel, M. Snuderl, A. Lussiez, L. Hiddingh, S. Mahmood, B. A. Tannous, A. F. Eichler, D. Fukumura, J. A. Engelman and R. K. Jain (2012). "Combined targeting of HER2 and VEGFR2 for effective 
treatment of HER2-amplified breast cancer brain metastases." Proc Natl Acad Sci U S A 109(45): E3119-3127.

Kute, T., C. M. Lack, M. Willingham, B. Bishwokama, H. Williams, K. Barrett, T. Mitchell and J. P. Vaughn (2004). "Development of Herceptin resistance in breast cancer cells." Cytometry A 57(2): 86-93.

Lampson, L. A. (2011). "Monoclonal antibodies in neuro-oncology: Getting past the blood-brain barrier." MAbs 3(2): 153-160.

Leyland-Jones, B. (2009). "Human epidermal growth factor receptor 2-positive breast cancer and central nervous system metastases." J Clin Oncol 27(31): 5278-5286.

Liebner, S., A. Fischmann, G. Rascher, F. Duffner, E. H. Grote, H. Kalbacher and H. Wolburg (2000). "Claudin-1 and claudin-5 expression and tight junction morphology are altered in blood vessels of human glioblastoma multiforme." Acta Neuropathol 100(3): 323-331.

Lin, N. U. (2013). "Breast cancer brain metastases: new directions in systemic therapy." ecancermedicalscience 7: 307.

Lin, N. U., J. R. Bellon and E. P. Winer (2004). "CNS metastases in breast cancer." J Clin Oncol 22(17): 3608-3617. 
Lockman, P. R., R. K. Mittapalli, K. S. Taskar, V. Rudraraju, B. Gril, K. A. Bohn, C. E. Adkins, A. Roberts, H. R. Thorsheim, J. A. Gaasch, S. Huang, D. Palmieri, P. S. Steeg and Q. R. Smith (2010). "Heterogeneous blood-tumor barrier permeability determines drug efficacy in experimental brain metastases of breast cancer." $\underline{\text { Clin }}$ Cancer Res 16(23): 5664-5678.

Loscher, W. and H. Potschka (2005). "Blood-brain barrier active efflux transporters: ATP-binding cassette gene family." NeuroRx 2(1): 86-98.

Mutlu, H. and A. Buyukcelik (2015). "The combination of weekly trastuzumab plus vinorelbine may be preferable regimen in HER-2 positive breast cancer patients with brain metastasis." J Oncol Pharm Pract 21(4): 310-312.

Palmieri, D., J. L. Bronder, J. M. Herring, T. Yoneda, R. J. Weil, A. M. Stark, R. Kurek, E. Vega-Valle, L. Feigenbaum, D. Halverson, A. O. Vortmeyer, S. M. Steinberg, K. Aldape and P. S. Steeg (2007). "Her-2 overexpression increases the metastatic outgrowth of breast cancer cells in the brain." Cancer Res 67(9): 41904198.

Palmieri, D., Q. R. Smith, P. R. Lockman, J. Bronder, B. Gril, A. F. Chambers, R. J. Weil and P. S. Steeg (2006). "Brain metastases of breast cancer." Breast Dis 26: 139147. 
Park, Y. H., M. J. Park, S. H. Ji, S. Y. Yi, D. H. Lim, D. H. Nam, J. I. Lee, W. Park, D. H. Choi, S. J. Huh, J. S. Ahn, W. K. Kang, K. Park and Y. H. Im (2009). "Trastuzumab treatment improves brain metastasis outcomes through control and durable prolongation of systemic extracranial disease in HER2-overexpressing breast cancer patients." Br J Cancer 100(6): 894-900.

Pestalozzi, B. C. and S. Brignoli (2000). "Trastuzumab in CSF." J Clin Oncol 18(11): 2349-2351.

Prabhakarpandian, B., M. C. Shen, J. B. Nichols, I. R. Mills, M. SidorykWegrzynowicz, M. Aschner and K. Pant (2013). "SyM-BBB: a microfluidic Blood Brain Barrier model." Lab Chip 13(6): 1093-1101.

Puhalla, S., W. Elmquist, D. Freyer, L. Kleinberg, C. Adkins, P. Lockman, J. McGregor, L. Muldoon, G. Nesbit, D. Peereboom, Q. Smith, S. Walker and E. Neuwelt (2015). "Unsanctifying the sanctuary: challenges and opportunities with brain metastases." Neuro Oncol 17(5): 639-651.

Rip, J., G. J. Schenk and A. G. de Boer (2009). "Differential receptor-mediated drug targeting to the diseased brain." Expert Opin Drug Deliv 6(3): 227-237.

Rostami, R., S. Mittal, P. Rostami, F. Tavassoli and B. Jabbari (2016). "Brain metastasis in breast cancer: a comprehensive literature review." J Neurooncol. 
Sanchez-Munoz, A., Y. Plata-Fernandez, M. Fernandez, A. Jaen-Morago, M. Fernandez-Navarro, C. de la Torre-Cabrera, C. Ramirez-Tortosa, J. Pascual, E. Alba and P. Sanchez-Rovira (2013). "Tumor histological subtyping determined by hormone receptors and HER2 status defines different pathological complete response and outcome to dose-dense neoadjuvant chemotherapy in breast cancer patients." $\underline{\text { Clin }}$ Transl Oncol.

Sendur, M. A., D. Uncu and N. Zengin (2014). "Longest progression-free survival with lapatinib and capecitabine combination followed by trastuzumab in HER2positive brain metastatic breast cancer." Med Oncol 31(4): 890.

Smith, Q. R. and Y. Takasato (1986). "Kinetics of amino acid transport at the bloodbrain barrier studied using an in situ brain perfusion technique." Ann N Y Acad Sci 481: 186-201.

Steeg, P. S., K. A. Camphausen and Q. R. Smith (2011). "Brain metastases as preventive and therapeutic targets." Nat Rev Cancer 11(5): 352-363.

Takasato, Y., S. I. Rapoport and Q. R. Smith (1984). "An in situ brain perfusion technique to study cerebrovascular transport in the rat." Am J Physiol 247(3 Pt 2): H484-493.

Tate, M. C. and M. K. Aghi (2009). "Biology of angiogenesis and invasion in glioma." Neurotherapeutics 6(3): 447-457. 
Tzeng, S. Y. and J. J. Green (2013). "Therapeutic nanomedicine for brain cancer." Ther Deliv 4(6): 687-704.

van Tellingen, O., B. Yetkin-Arik, M. C. de Gooijer, P. Wesseling, T. Wurdinger and H. E. de Vries (2015). "Overcoming the blood-brain tumor barrier for effective glioblastoma treatment." Drug Resist Updat 19: 1-12.

Vaz-Luis, I., R. A. Ottesen, M. E. Hughes, P. K. Marcom, B. Moy, H. S. Rugo, R. L. Theriault, J. Wilson, J. C. Niland, J. C. Weeks and N. U. Lin (2012). "Impact of hormone receptor status on patterns of recurrence and clinical outcomes among patients with human epidermal growth factor-2-positive breast cancer in the National Comprehensive Cancer Network: a prospective cohort study." Breast Cancer Res 14(5): R129.

Villanueva, M. T. (2013). "Drug therapy: Smuggling trastuzumab into the brain." Nat Rev Clin Oncol 10(12): 669-669.

Witzel, I., L. Oliveira-Ferrer, K. Pantel, V. Muller and H. Wikman (2016). "Breast cancer brain metastases: biology and new clinical perspectives." Breast Cancer Res 18(1): 8 .

Yeh, R. H., J. C. Yu, C. H. Chu, C. L. Ho, H. W. Kao, G. S. Liao, H. W. Chen, W. Y. Kao, C. P. Yu, T. Y. Chao and M. S. Dai (2015). "Distinct MR Imaging Features of 
Triple-Negative Breast Cancer with Brain Metastasis." J Neuroimaging 25(3): 474481.

Yonemori, K., K. Tsuta, M. Ono, C. Shimizu, A. Hirakawa, T. Hasegawa, Y. Hatanaka, Y. Narita, S. Shibui and Y. Fujiwara (2010). "Disruption of the blood brain barrier by brain metastases of triple-negative and basal-type breast cancer but not HER2/neu-positive breast cancer." Cancer 116(2): 302-308.

Zimm, S., G. L. Wampler, D. Stablein, T. Hazra and H. F. Young (1981). "Intracerebral metastases in solid-tumor patients: natural history and results of treatment." Cancer 48(2): 384-394. 


\section{CHAPTER 5}

\section{CONCLUSIONS AND FUTURE DIRECTIONS}

\subsection{Conclusions}

This dissertation set out to study the distribution and efficacy of chemotherapeutics in a novel, microfluidic, in vitro preclinical model of brain metastases of breast cancer. Chapter 2 is an in depth review of the current static and microfluidic in vitro models most commonly available to researchers. This review shows that a new, commercially available model that mimics in vivo is drastically needed for the advancement of blood-brain barrier (BBB) and blood-tumor barrier (BTB) research. This review article further set out to evaluate the performance of cell-based in vitro systems of the BBB. It was found to be very difficult to evaluate the performance of the plethora of different models used because of the lack of coherence in the research field. The authors therefore call for a more standardized method to evaluate the performance of the different in vitro systems developed as an effort to jointly bring the research field forward. In pursuit of a widely-applicable, cost-effective, and accurate in vitro model of the BBB and BTB at the brain, all of the discussed microfluidic evices must be taken into consideration.

In chapter 3, we set out to validate a novel and dynamic microfluidic in vitro BBB model. Through various permeability studies, we found that the permeability of large molecule dextrans, as well as small molecule dextrans, and Rhodamine-123 (with and 
without inhibitors) were characteristic and relatable to what is seen in vivo. The added shear stress exerted on the endothelial cells from the addition of flow eliminated the unstirred water layer and allowed for different tracers to be added and real-time followed from outer (apical) to central (basolateral) compartment. P-glycoprotein (p-gp) was also shown to be functional and intact in both BBB and BTB models, and relatable to current in vivo data. We found that this novel microfluidic in vitro device successfully mimicked the in vivo barrier in regard to shear stress, permeability, and efflux. Based on these characteristics, this microfluidic chip shows potential for use in BBB and BTB research.

When it comes to drug and antibody distribution across the BBB and BTB, the literature is conflicting and this topic continues to be highly debated. Despite the partial breakdown of the barrier, the BTB still exhibits efflux transporters (and other BBB properties) to a highly functioning degree, causing difficulties in the delivery of most drugs. The aim of Chapter 4 was to test the distribution of trastuzumab in both an in vivo and in vitro model. This chapter demonstrates that, though in minute quantities, trastuzumab does in fact cross the blood-brain and blood-tumor barriers. The heterogeneity of the antibody dispersion observed within the in vitro tumors mimics that seen in the in vivo tumor model. Expanding on these data, future work should include the use of additional antibodies used to treat brain metastases of breast cancer, as well as the addition of different small molecule inhibitors. 


\subsection{Future Directions}

Throughout this dissertation, specifically during the validation of the microfluidic chip in chapter 3 , multiple obstacles occurred throughout the experiments, leading to months of troubleshooting and the redesigning of projects. However, due to these obstacles, we have worked diligently with the CFD Research Corporation to develop, bioengineer, and restructure multiple variations to the current microfluidic chip. The proposed bioengineering modifications (Fig. 5.1) will allow for a greater diversification in the types of experiments that will be able to be run through this chip, as well as helping validate this in vitro model even further.

\subsubsection{Engineering Modifications}

The biggest limitation with the current microfluidic chip is the inability to measure transendothelial electrical resistance (TEER) values across the different barriers. TEER is a quantitative technique used to measure integrity of the BBB and BTB, and allows for comparison between models. In vivo, the TEER of brain capillaries is $\sim 2,000$ ohm* $\mathrm{cm}^{2}$ (Crone and Christensen 1981, Olesen and Crone 1983), where as the TEER of the standard in vitro static BBB transwell is $25 \Omega \mathrm{cm}^{2}$ (Booth and Kim 2012). We have worked thoroughly with CFD Research Corporation to redesign their microfluidic device to adequately measure real time TEER in a way as to not disrupt the cell culture conditions. In order to measure TEER, three different components are required; 1) ports for insertion of electrodes, 2) development of electrodes and 3) portable and costeffective instruments for real-time monitoring. Figure 5.2 shows a conceptual schematic of the design for TEER measurement with the circles indicating location for electrodes 
insertion. Every in vitro model has the ability for a TEER value to be measured, allowing for the comparison of the tightness of that model's barrier to in vivo so with this bioengineering modification, the ability of this microfluidic chip to create an substantial and significant $\mathrm{BBB}$ will be able to be measured and directly compared to all other in vitro, and in vivo, models.

Initially, when the projects for Chapter 3 began, we incorporated the use of five different compounds, including small and large molecules as well as a couple radiolabeled chemotherapeutics to add to the validation of this model in regards to drug movement across the BBB and BTB. When we began to run the experiments, a limitation that we observed was the very small size of the recovery ports. This limited the recovery of radiolabeled compounds and consequently we could not complete analysis. In the current design of the chip, the entire apical chamber, from the input to the recovery port only holds $0.806 \mu$, with the central chamber holding only $1.795 \mu 1$ (Fig. 5.3). Because of this limitation, we began to formulate the basis for a modified chip with larger port and chamber volumes, in order to run radioactive experiments. The proposed CAD layout of the optimized design and parameters of the modified chip is shown in Figure 5.4. The modifications include the changing of the circular central chamber to an elongated chamber design, and lengthening the overall device, allowing it to span the entire microscope glass slide. With these modifications, perfusate samples will to $10-20 \mu 1$ of sample for perfusate analysis, allowing for the integration of a vast amount of varying compounds and drugs. 
The last engineering modification to this device is to modify the device in order to isolate metastatic cells with varying metastatic potential. The mechanism of tumor cell extravasation is unknown and has not been successfully studied in vitro. The extravasation of tumor cells has been previously shown with this microfluidic device (Fig 5.5), though the mechanism has not been elucidated, due in part to the inability to retrieve individual, or selective, tumor cells from the device. The first step for future modification is to alter the central chamber to 1) allow for quantification of cells based upon metastatic potential and 2) be able to collect cells with varying metastatic potential for next generation sequencing. One of the most exciting aspects of this modification is that the genomic driving forces of BBB cancer cell extravasation would be understood through the experiments using next generation sequencing of cells that can cross the BBB endothelia. In order to isolate the cells for single cell populations, the system will be redesigned as shown in Figure 5.6. The output of the central chambers will be distributed into multiple channels to separate cells with different metastatic potential. Each of the channels will be injected into a sample tube for isolation and analysis. Figure 5.6 also shows examples of fluidic isolation for separation based on the outlets. This modification has the potential to lead to significant insights and potentially druggable targets for cancer cells invading brain, something that has not yet been studied.

\subsubsection{Biological Modifications}

Throughout my project I utilized Human Umbilical Vein Endothelial Cells (HUVECs) as my endothelial cells of choice due to their ability to be used until a high cell culture passage, and the relative ease in the culturing ability. However, it was 
brought to our attention that HUVECs may not be the best model to use in vitro to mimic the in vivo $\mathrm{BBB}$. So to address this, we have postulated modifications in the device to improve cell culture conditions. Within these modifications, it might be of interest to utilize Dr. Eric Shusta's model. Shusta utilizes human pluripotent stem cells that are simultaneously co-differentiated to both neural and endothelial lineages. By doing this, these cells produce an embryonic brain-like in vitro micro-environment that generates human pluripotent stem cells-brain microvascular endothelia cells. These cells would be a great cell to use for recreating the blood-brain or blood-tumor barriers because these cells have been shown to possess BBB endothelial characteristics such as well-developed tight junctions, high TEER, low passive permeability, and active and polarized efflux transporters (Perriere, Yousif et al. 2007, Lippmann, Azarin et al. 2012), all characteristics that are important in the in vivo BBB.

In addition to modifying the endothelial cells, other biological modifications can be utilized to further verify the model through the use of different brain metastases cells (human pluripotent stem cells, patient derived xenografts (PDXs), JimtBr cells and MDA-MB-231Br cells) to create various different brain microenvironments, as well as the study of the permeability of each cell line in vitro and to be able to compare to the relative in vivo data. As the incidence of brain metastases of breast cancer continues to increase, the need for affordable, accessible, and accurate portrayals of the in vivo BBB in the form of in vitro models also increases. It is our hope that through these modifications, these microfluidic chips will become a widely accepted in vitro model, 
meeting and exceeding all of the aforementioned desires of a clinically relevant in vitro model. 


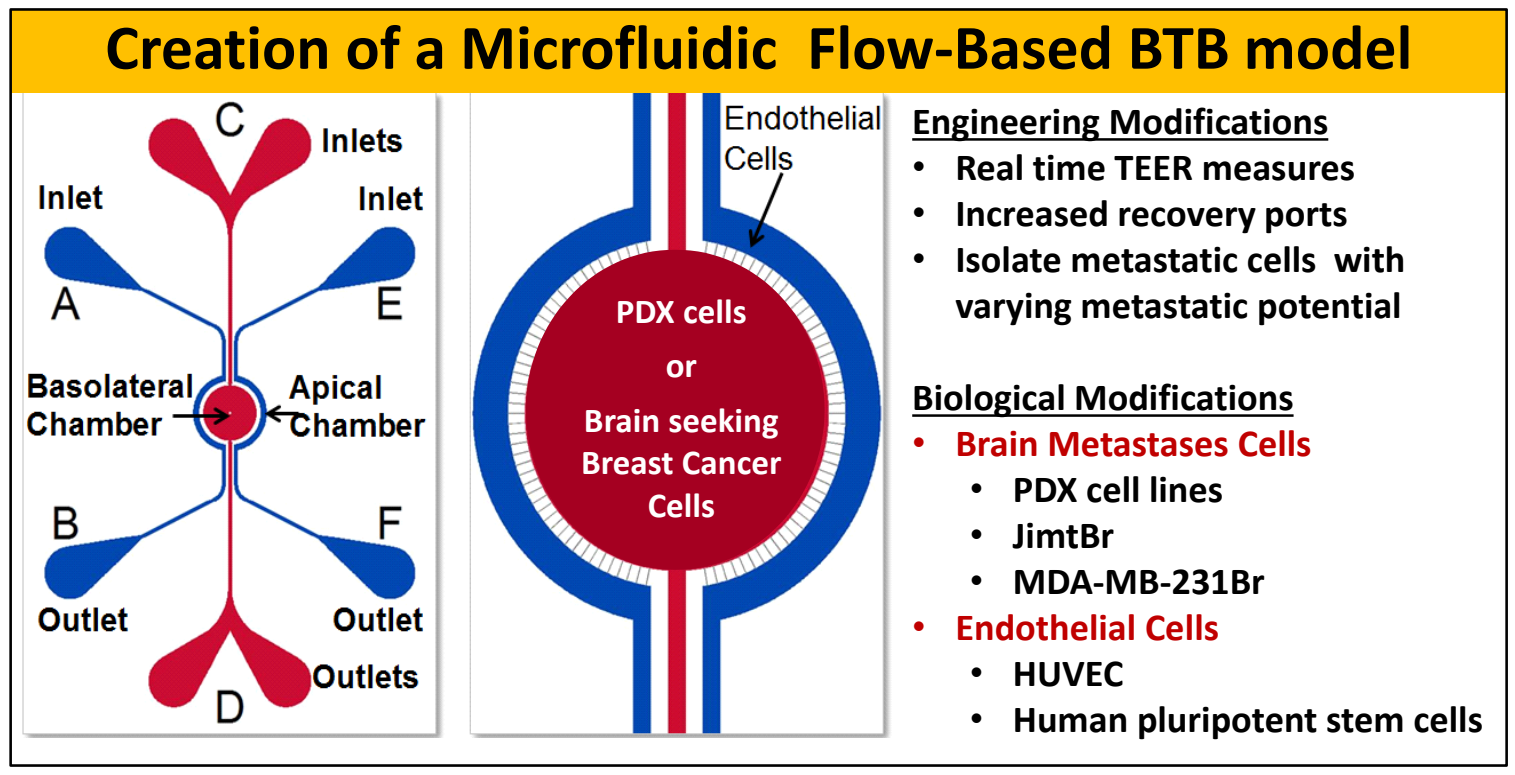

Figure 5.1: Microfluidic BTB device overall modifications

Inlet ports where perfusion flow enters outer chamber (A) and central chamber (C).

Apical chamber contains endothelial cells that communicate across $3 \mu \mathrm{m}$ pores with metastatic cancer cells in the central chamber. Outlet ports where perfusate from the outer compartment (F) and central compartments are collected (D). 


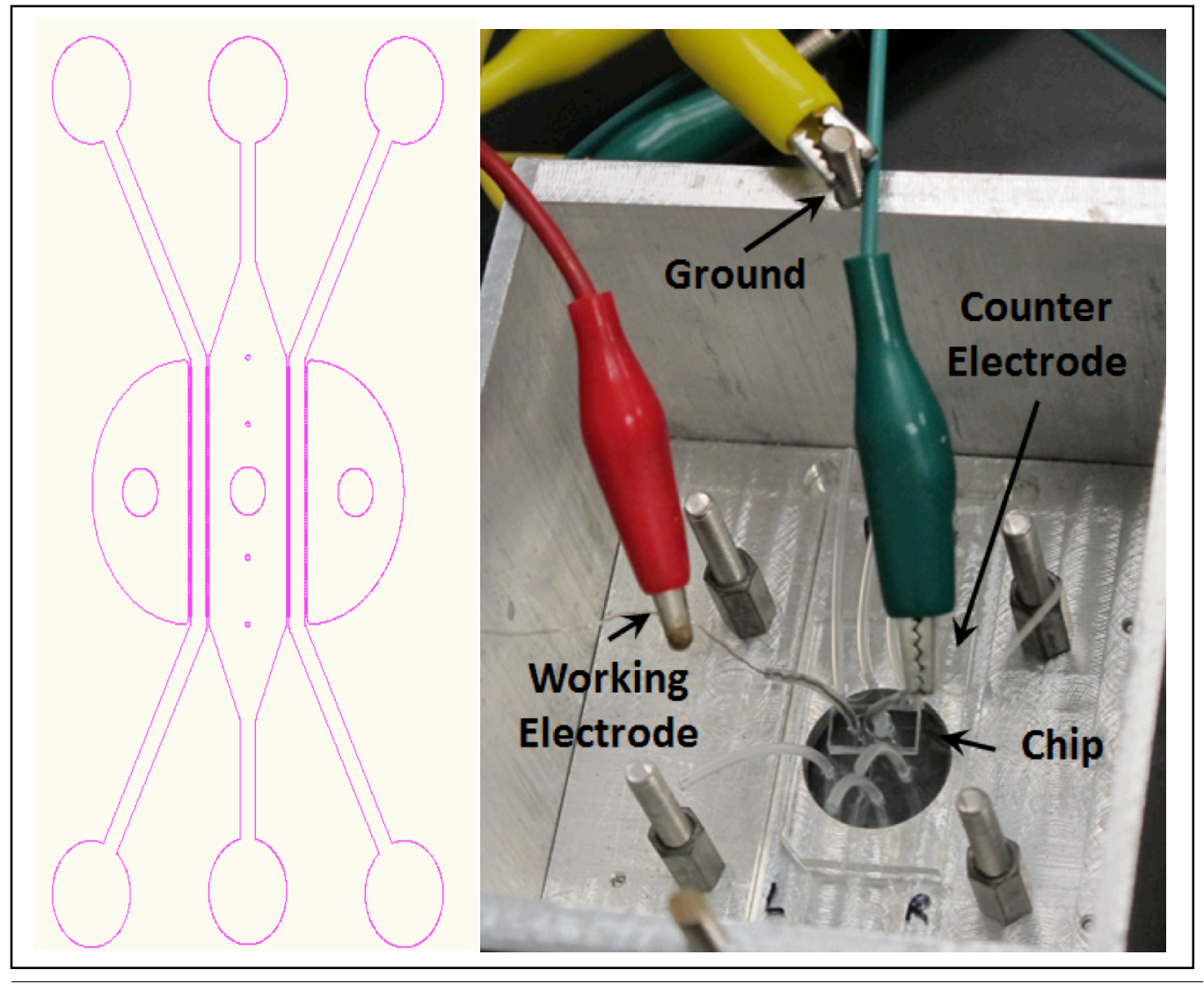

Figure 5.2: Re-engineering of the BTB microfluidic device to allow for real-time TEER measurements

A schematic (Left) and an example set-up of the device for TEER measurement is shown (right). Silver chloride electrodes are threaded through Tygon tubing attached to the electrode ports. An electrochemical workstation is interfaced using clips. 


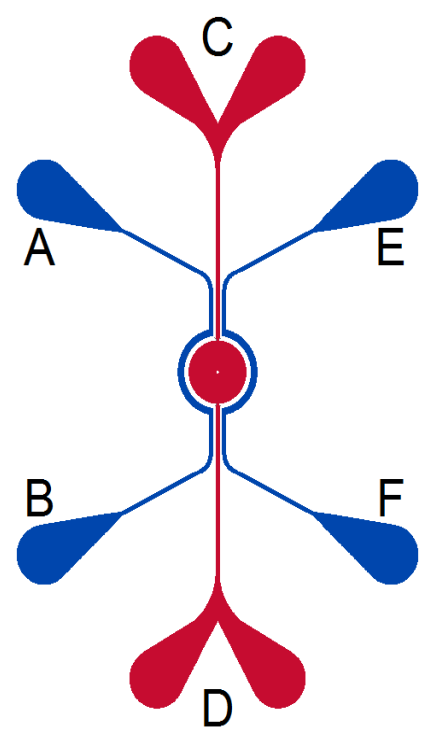

$$
\begin{aligned}
& \text { Port A to Port B } \\
& \text { Volume (same } \\
& \text { as } E \text { to } F)= \\
& 0.806 \text { ul } \\
& \text { Port C to Port D } \\
& \text { Volume }=1.795 \\
& \text { ul } \\
& \text { Total device } \\
& \text { volume } \\
& =3.409 \text { ul }
\end{aligned}
$$

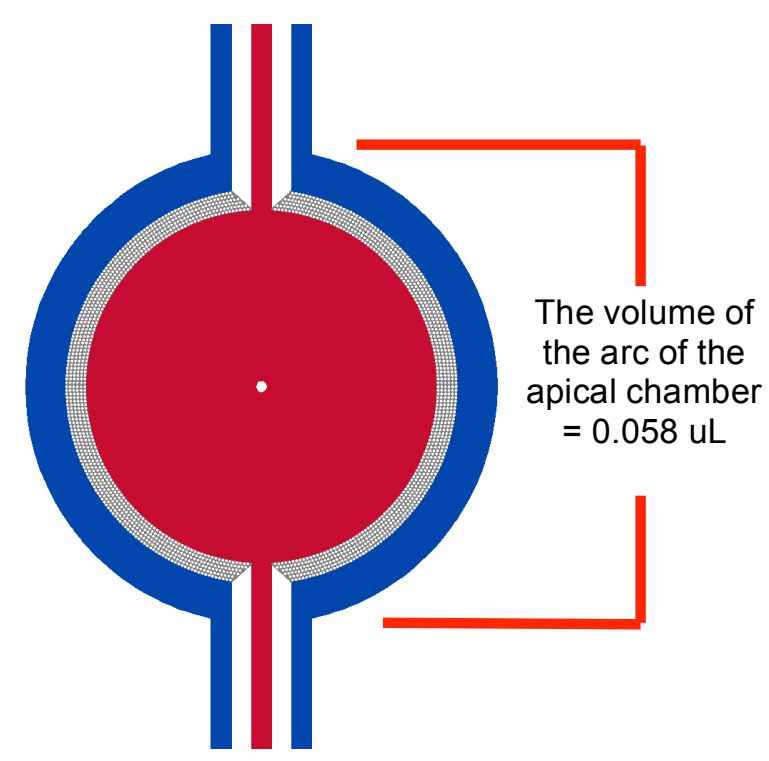

Figure 5.3: Port volumes of the current microfluidic device

Inlet ports for the apical chambers $(\mathrm{A}, \mathrm{E})$, basolateral chamber $(\mathrm{C})$ and outlet ports for the apical chambers $(\mathrm{B}, \mathrm{F})$ and basolateral chamber (D) are depicted with the respected volumes described. 


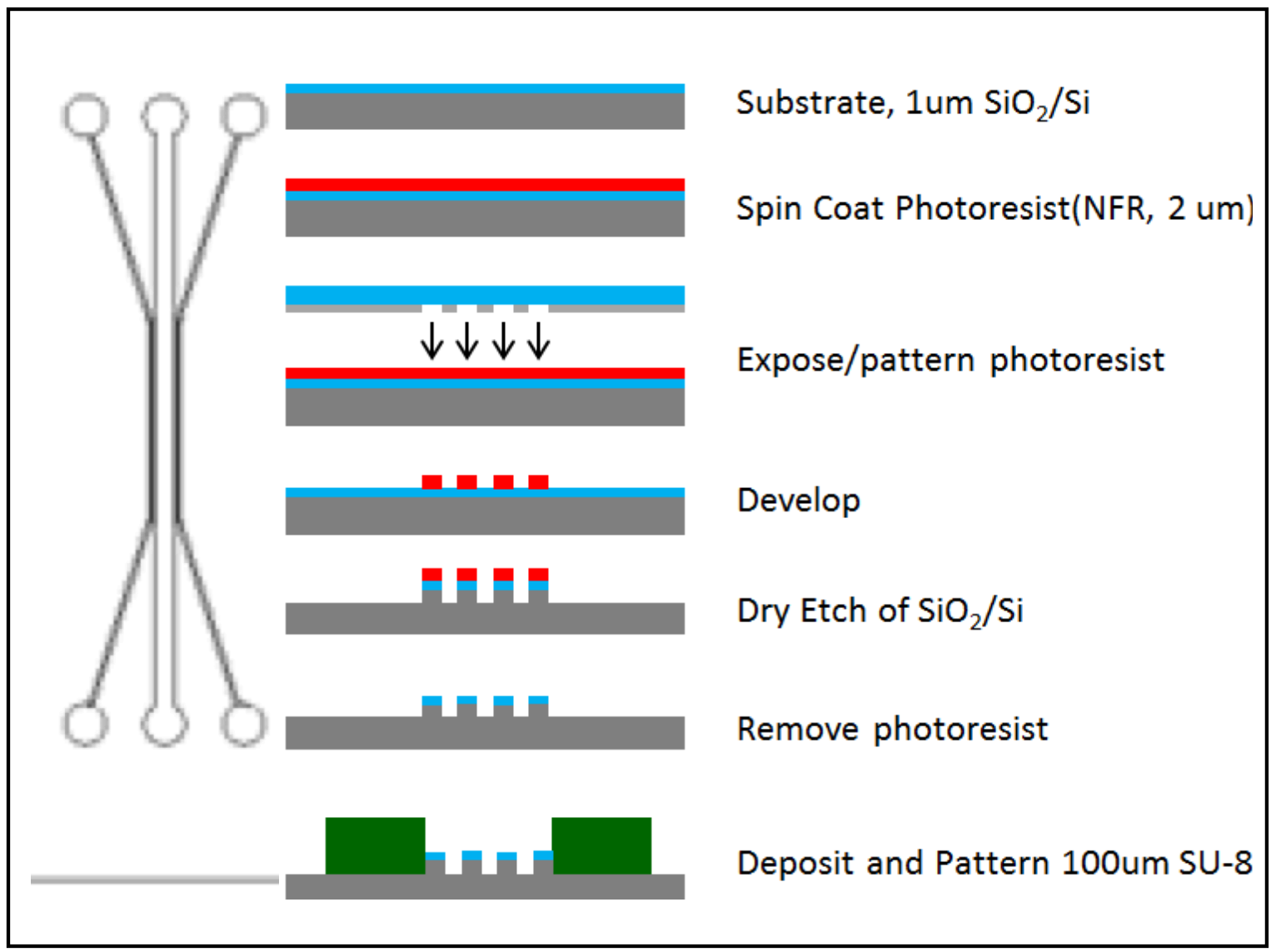

Figure 5.4: Proposed redesign for increased recovery port volumes of the microfluidic device

A CAD layout of the design and parameters for the optimized BTB microfluidic device with the proposed redesign schematic of the BTB microfluidic device (Left) and the process of microfabrication of the porous architecture (Right). 


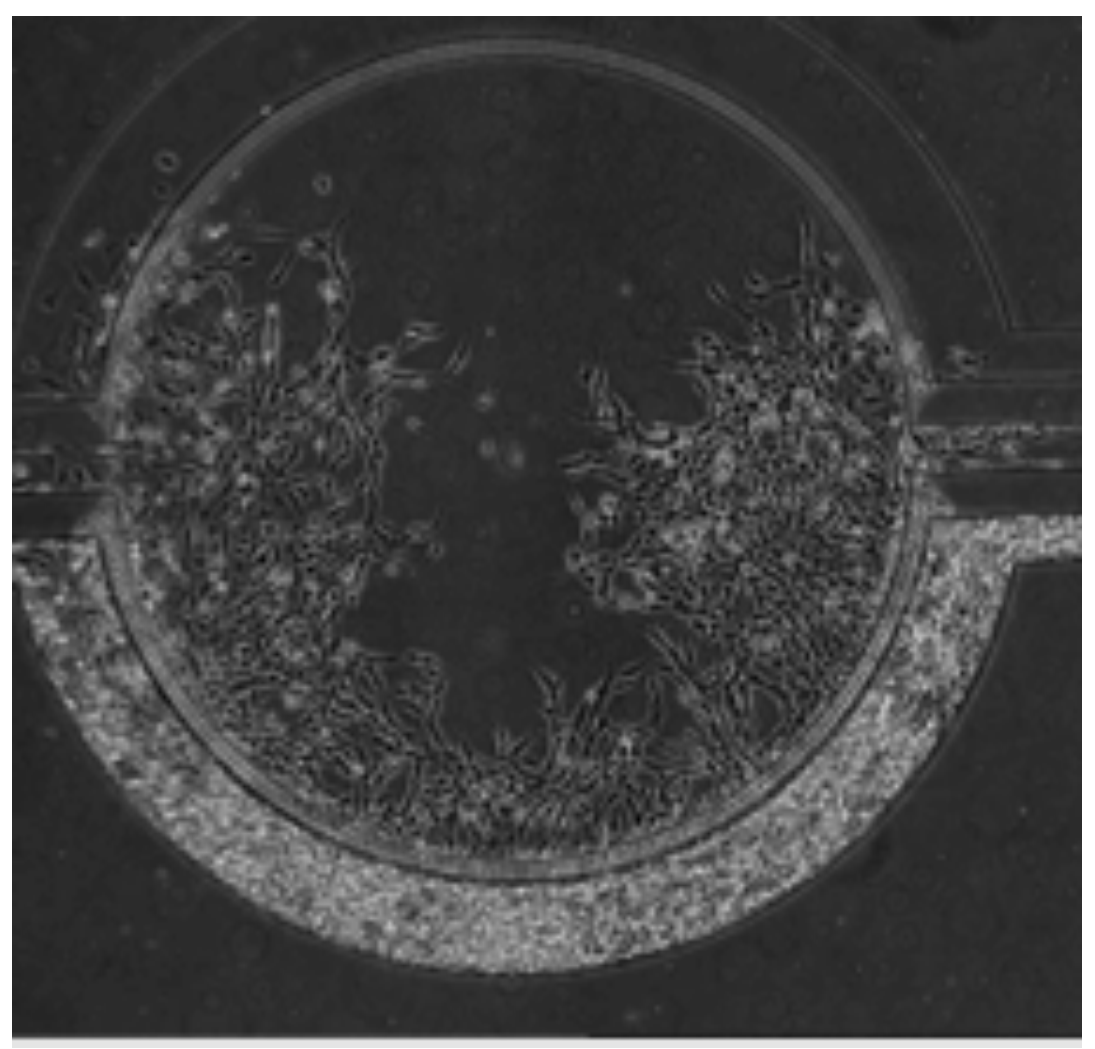

Figure 5.5: Tumor cell extravasation

Tumor cell extravasation across a microfluidic device. 


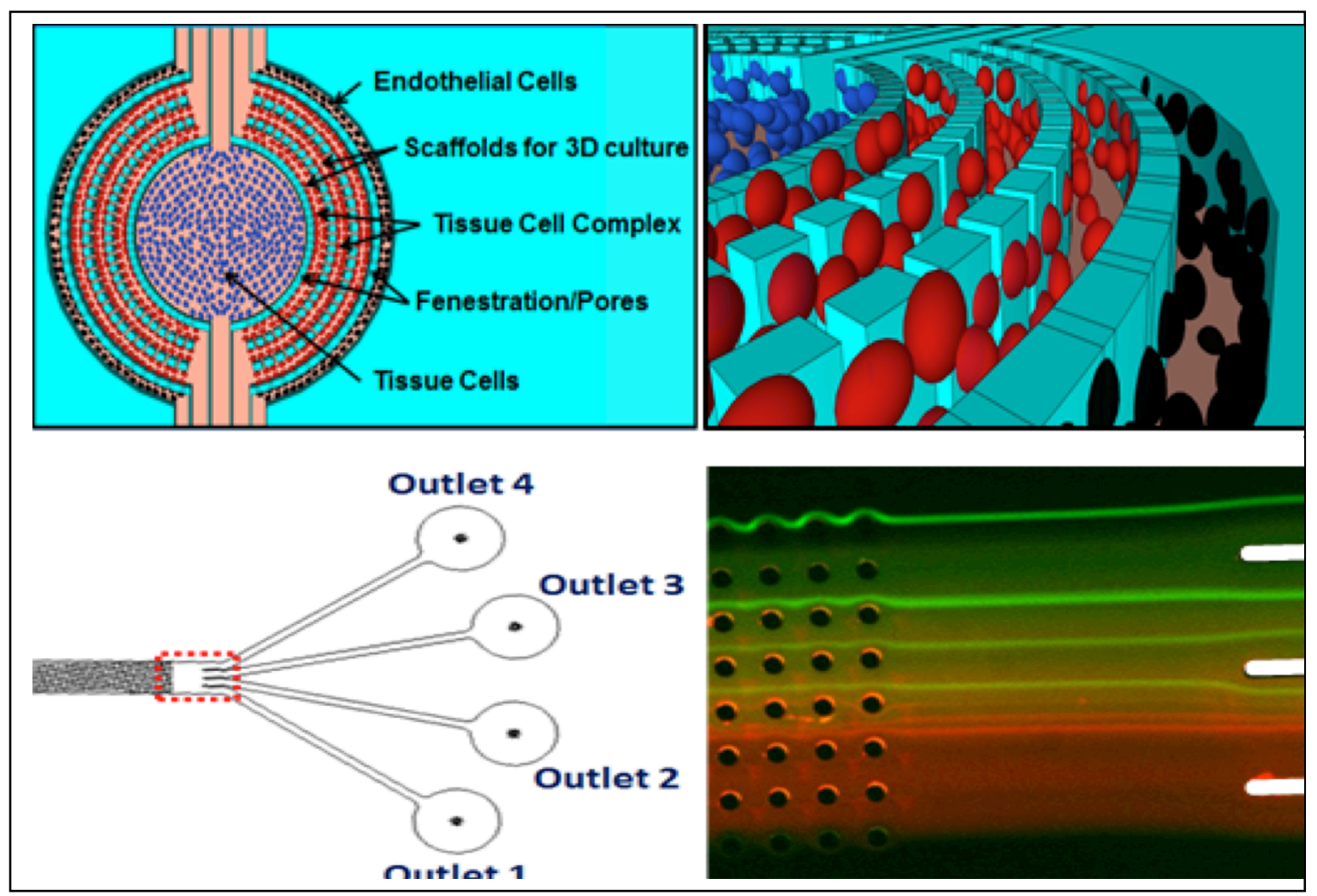

Figure 5.6: Isolation of different cells of varying metastatic potential

Concept of multi-cellular architectures in the central chamber which can separate invading cells by degree of migration (top). Concept of multiple outlets from the central chamber to capture the different cell populations. 


\subsection{References}

Booth, R. and H. Kim (2012). "Characterization of a microfluidic in vitro model of the blood-brain barrier (muBBB)." Lab Chip 12(10): 1784-1792.

Crone, C. and O. Christensen (1981). "Electrical resistance of a capillary endothelium." J Gen Physiol 77(4): 349-371.

Lippmann, E. S., S. M. Azarin, J. E. Kay, R. A. Nessler, H. K. Wilson, A. Al-Ahmad, S. P. Palecek and E. V. Shusta (2012). "Derivation of blood-brain barrier endothelial cells from human pluripotent stem cells." Nat Biotechnol 30(8): 783-791.

Olesen, S. P. and C. Crone (1983). "Electrical resistance of muscle capillary endothelium." Biophys J 42(1): 31-41.

Perriere, N., S. Yousif, S. Cazaubon, N. Chaverot, F. Bourasset, S. Cisternino, X. Decleves, S. Hori, T. Terasaki, M. Deli, J. M. Scherrmann, J. Temsamani, F. Roux and P. O. Couraud (2007). "A functional in vitro model of rat blood-brain barrier for molecular analysis of efflux transporters." Brain Res 1150: 1-13. 


\section{CURRICULUM VITAE}

\section{PERSONAL INFORMATION}

Tori B. Terrell-Hall, B.S.
ADDRESS AND CONTACT INFORMATION:
HOME:
310 Parkwood Lane
Levelland, TX 79336
Cell: (806) 292-7314
Email: torithall@gmail.com
WORK:
West Virginia University
School of Pharmacy
Department of Pharmaceutical Sciences
1 Medical Center Dr.
BMRF Lab 170
Morgantown, WV 26506
Phone: (304)581-1794
Email: tbterrell@mix.wvu.edu

\section{EDUCATION}

\section{INSTITUTION}

West Virginia University

School of Pharmacy,

Department of Pharmaceutical

Sciences

Morgantown, WV 26506

Texas Tech University,

Health Sciences Center,

Graduate School of Biomedical

Sciences

Amarillo, TX 79106

West Texas A\&M University

Canyon, TX 79015

\section{DEGREE/POSITION}

Ph.D., Pharmaceutical and

Pharmacological Sciences

\section{YEAR}

Jan 2014 -

Dec 2016
Ph.D., Pharmaceutical

Sciences
Aug 2012 -

Dec 2013
B.S.

Biology \& Chemistry
Aug 2007 -

May 2011 


\section{HONORS AND AWARDS}

$04 / 2015$

$08 / 2014-05 / 2015$

$04 / 2011$

$08 / 2010-05 / 2011$
Rho Chi National Pharmacy Honor Society Induction, West Virginia University

Chair-Elect, West Virginia University student chapter of AAPS, West Virginia University

Beta Beta Beta Biological National Honor Society, Induction, West Texas A\&M University

Treasurer, Pre-Healthcare Club, West Texas A\&M

University

\section{WORK EXPERIENCE}

01/2014 - Current

Lab Manager - (Department of Pharmaceutical Sciences, School of Pharmacy, West Virginia University, Morgantown, WV). Provide administrative support for the lab through resolving problems, managing employee issues and conflicts, and communicating important trainings and regulatory regulations to the lab. Plan, budget, and order new equipment and compatible software. Oversee lab equipment performances and overall lab productivity. Maintain laboratory supplies inventory by placing orders and sending receipts and invoices to department finance 
officer. Manage undergraduate, graduate, and professional student's projects and experiments.

01/2014 - Current

$08 / 2012-12 / 2013$

$01 / 2012-08 / 2012$

\section{Research Assistant - Dr. Paul Lockman's Lab}

(Department of Pharmaceutical Sciences, School of

Pharmacy, West Virginia University, Morgantown, WV).

Design, conduct, and analyze experiments. Design, write, edit, and review manuscripts.

\section{Research Assistant - Dr. Paul Lockman's Lab}

(Department of Pharmaceutical Sciences, School of Pharmacy, Texas Tech University Health Sciences Center, Amarillo, TX). Designed, conducted, and analyzed experiments. Became proficient in in vitro and in vivo blood-brain barrier models. Designed, wrote, edited, and reviewed manuscripts. Oversaw the ordering for the lab. Managed, communicated, and oversaw the packing of, and moving of, mentor's lab to new university.

Lab Technician - (Department of Pharmaceutical Sciences, School of Pharmacy, Texas Tech University Health Sciences Center, Amarillo, TX). Observed and 
became proficient in various lab techniques. Managed and ordered supplies for the lab.

$03 / 2011-05 / 2011$

Cell Culture Technician - (Department of Life, Earth, and Environmental Sciences, West Texas A\&M University, Canyon, TX). Observed and became proficient in molecular and cellular biology techniques, proper aseptic technique, and sterilization techniques.

$05 / 2008-08 / 2008$

Data Technician - (Baylor Medical Pavilion, Dallas, TX). Observed, compiled, and arranged patient data in publishable figures and graphs regarding current and former patients receiving Infliximab treatment.

\section{CLASSROOM TEACHING EXPERIENCE}

01/2014 - Current

$01 / 2016-05 / 2016$
PHAR 796. Graduate Seminar. Deliver lectures to faculty, graduate, professional, and undergraduate students on CNS and translational pharmacology research. West Virginia University, School of Pharmacy.

PHAR 814. Biochemical Pharmacology. Responsible for directing discussion of first year pharmacy students in 
application of biochemical pharmacology within case study groups, and designing rubrics to score and evaluate students. West Virginia University, School of Pharmacy.

$01 / 2013-03 / 2013$

\section{GPSC 5211. Drug Delivery Systems Part II, Graduate}

Pharmaceutics II. Taught 6 weeks of pharmaceutics lab to Pharm.D. students, focusing on the formulation of medicated lip balm, lozenges, and suckers. Texas Tech University Health Science Center, School of Pharmacy.

\section{RESEARCH TEACHING EXPERIENCE}

01/2014 - Current

Mentorship. Mentoring, supervising, teaching, and training of undergraduate, graduate, and professional students in molecular, cellular, in vitro, in vivo, and oncology based biology research, experimental design, implementation, and analysis. Total: Undergraduate students, 3. Graduate students, 3. Pharm.D. students, 4. West Virginia University, School of Pharmacy.

$08 / 2012-12 / 2013$

Mentorship. Mentored, supervised, taught, and trained graduate students in molecular, cellular, in vitro, in vivo, and oncology based biology research, experimental design, 
implementation, and analysis. Total: Graduate students, 2.

Texas Tech University Health Sciences Center, School of

Pharmacy.

\section{SCIENTIFIC EXPERIENCE}

- Extensive expertise in various molecular biology and biochemical techniques including, but not limited to:

- Protein labeling, DNA preparation / RNA sequencing, cell culture, mammalian and cancer cell transfection, flow cytometry, immunohistochemistry (IHC), immunofluorescence chemistry (IFC), and scintillation counting.

- Histologic processing, cryopreservation, and sectioning of various tissues and organs.

- Technical experience in working with mammalian, cancer, and stem cell lines.

- Extensive experience in the following imaging techniques:

- Confocal, brightfield, fluorescence, multiphoton, inverted live cell confocal, dual upright microscope and stereoscope, laser microdisection scope, and transmission electron microscopes, as well as Nikon NIS 
elements and analysis, 3i SlideBook, and other imaging analysis software packages.

- Extensive experience in chemotherapy, specifically in brain metastases of breast cancer.

- Extensive experience with in vitro models (transwells, BBB chip models) and in vivo mouse models to study the movement and efficacy of chemotherapy in the treatment of brain metastases of breast cancer.

- Extensive expertise in radiopharmaceutical handling and autoradiography to determine drug concentration or distribution.

- Experience with in-situ brain perfusion kinetics and in vitro BBB model kinetics.

- Demonstrated strong leadership in managing various research projects within the lab and among various coworkers.

\section{PROFESSIONAL ACTIVITIES}

\section{PROFESSIONAL ORGANIZATION MEMBERSHIPS}

- American Association for Cancer Research, AACR (2015 - current)

- American Association of Pharmaceutical Scientists, AAPS (2014 - current) 


\section{PROFESSIONAL PUBLICATIONS - PEER REVIEWED}

Terrell-Hall TB, Ammer AG, Griffith JIG, and Lockman PR. (2016) Permeability Across the Blood-Brain Barrier and Blood-Tumor Barrier; A Novel In vitro Model on a Chip. Cancer Growth Metastasis. Submitted.

Terrell-Hall TB, Amrawey FE, Nounou MI, and Lockman PR. (2016) Trastuzumab efficacy in an in vivo and in vitro model of brain metastases of breast cancer. Fluids Barriers CNS. Submitted.

Bohn KA, Adkins, CE, Mittapalli RK, Terrell-Hall TB, Mohammad AS, Shah N, Dolan EL, Nounou MI, Lockman PR. (2016) Semi-automated rapid quantification of brain vessel density utilizing fluorescent microscopy. J Neurosci Methods. 1;270:124-31.

Adkins CE, Mohammad AS, Terrell-Hall TB, Dolan EL, Shah N, Sechrest E, Griffith J, Lockman PR. (2016) Characterization of passive permeability at the blood-tumor barrier in five preclinical models of brain metastases of breast cancer. Clin Exp Metastsis. 33(4):373-83.

Adkins CE, Nounou MI, Hye T, Mohammad AS, Terrell-Hall T, Mohan NK, Eldon MA, Hoch U, Lockman PR. (2015) NKTR-102 Efficacy versus irinotecan in a mouse model of brain metastases of breast cancer. BMC Cancer. 15:685 
Adkins CE, Nounou MI, Mittapalli RK, Terrell-Hall TB, Mohammad AS, Jagannathan R, Lockman PR. (2015) A novel preclinical method to quantitatively evaluate early-stage metastatic events at the murine blood-brain barrier. Cancer Prev Res. 8(1):68-76.

El-Habashy SE, Nazief AM, Adkins CE, Wen MM, El-Kamel AH, Hamdan AM, Hanafy AS, Terrell TO, Mohammad AS, Lockman PR, Nounou MI. (2014) Novel treatment strategies for brain tumors and metastases. Pharm Pat Anal. 3(3):279-96.

Adkins CE, Mittapalli RK, Manda VK, Nounou MI, Mohammad AS, Terrell TB, Celik Y, Groethe TR, Lockman JA, Lockman PR. (2013) P-glycoprotein mediated efflux limits substrate and drug uptake in a preclinical brain metastases of breast cancer model. Frontiers in Pharmacology. 4:136.

Mittapalli RK, Liu X, Adkins CE, Nounou MI, Bohn KA, Terrell TB, Qhattal HS, Geldenhuys WJ, Palmieri D, Steeg PS, Smith QR, Lockman PR. (2013) Nanoparticle Paclitaxel Conjugates Prolong Overall Survival in a Preclinical Brain Metastases of Breast Cancer Model. Mol Canc Ther. 12(11): 2389-99.

\section{BOOK CHAPTERS}

Nounou MI, Adkins CE, Terrell TB, Bohn KA, Lockman PR. 2012. Drug Delivery to the CNS: Breaking Down the Barrier. In Book "Drug Delivery” (Edited by. AK Mitra) Jones and Bartlet Learning, Burlington MA. ISBN - 10: 1284025683, ISBN-13: 978$1284025682,2014$. 


\section{PUBLISHED ABSTRACTS}

Mohammad AS, Adkins CE, Terrell-Hall TB, Sechrest, ER, Dolan E, Griffith J, Shah N, Jagannatha R, Lockman PR. "Demonstration of casual relationship between blood-tumor barrier permeability changes and chemotherapeutic uptake and effect in brain micrometastases of breast cancer.” AACR Annual Meeting. New Orleans, LA. 2016.

Adkins CE, Mohammad AS, Dolan E, Griffith J, Terrell-Hall T, Lockman PR.

"Inhibition of TGFß to prevent brain metastases of breast cancer." AACR Annual Meeting. New Orleans, LA. 2016.

Bohn K, Terrell-Hall TB, Lockman PR, et al. "Vascular remodeling is associated with increased permeability of experimental brain metastases of breast cancer.” AACR Annual Meeting. Philadelphia, PA. 2015.

Bohn K, Sechrest ER, Adkins CE, Mittapalli RK, Nounou MI, Terrell-Hall TB, Mohammad AS, Lockman PR. "Inhibition of VEGF and angiopoietin-2 to reduce brain metastases of breast cancer burden.” AACR Annual Meeting. Philadelphia, PA. 2015. Mohammad AS, Adkins CE, Mittapalli RK, Terrell-Hall TB, Nounou MI, Lockman PR. "Characterization of changes in passive permeability and drug uptake at the blood-tumor barrier in four preclinical models of brain metastases of breast cancer." AACR Annual Meeting. Philadelphia, PA. 2015. 
Nounou MI, Adkins CE, Terrell TO, Mohamed A, Vitalis T, Gabathuler R, and Lockman PR. "Anti-cancer antibody trastuzumab-melanotransferrin conjugate (BT2111) for the treatment of metastatic HER2+ breast cancer tumors in the brain: An in vivo study"; Poster Presentation, The American Association of Cancer Research (AACR) annual meeting, San Diego, CA, USA, April 5-9, 2014.

Nounou MI, Hoch U, Adkins CE, Terrell TO, Villalba H, Eldon ME, and Lockman PR. "Etirinotecan pegol accumulates in breast cancer brain metastases and prolongs survival in an experimental model of brain metastases of human triple negative breast cancer"; Poster Presentation, The American Association of Cancer Research (AACR) annual meeting, San Diego, CA, USA, April 5-9, 2014.

Nounou MI, Adkins CE, Terrell TB, Villalba H, and Lockman PR. "Characterization of changes in passive permeability and drug uptake at the blood-tumor barrier in four preclinical models of brain metastases of breast cancer"; Poster Presentation, The American Association of Pharmaceutical Scientists (AAPS) annual meeting, San Antonio, TX, USA, November 10-14, 2013.

Hoch U, Nounou MI, Adkins CE, Terrell TB, Villalba H, Eldon ME, Perez E, and Lockman PR. "Etirinotecan pegol prolongs survival in an experimental model of brain metastasis of human triple negative breast cancer"; Poster Presentation, European Cancer Congress 2013 (ECCO-ESMO-ESTRO), Amsterdam, Netherlands, September 27thOctober 1st 2013. 
Gabathuler R, Vitalis TZ, Nounou MI, Iqbal U, Moreno M, Adkins CE, Terrell TO, Smith QR, Jefferies WA, and Lockman PR; " BT2111, a New Anti-Cancer Agent composed of trastuzumab and Transcend a Vector for Brain Delivery for the Treatment of Metastatic Her2+ Breast Cancer"; Poster Presentation, AACR-NCI-EORTC International Conference on Molecular Targets and Cancer Therapeutics, Boston, MA, USA, October 19-23, 2013.

Hoch U, Nounou M, Adkins CE, Terrell TB, Villalba H, Eldon E, Lockman PR. "Etirinotecan pegol prolongs survival in an experimental model of brain metastasis of human triple negative breast cancer". 17th ECCO - 38th ESMO, Amsterdam, Netherlands. 2013.

Nounou MI, Bohn KA, Adkins CE, Terrell TB, Bansal A, Smith QR, Lockman PR. "Effect of Bevacizumab on vascular permeability and drug uptake in brain metastases of breast cancer". Annual Meeting of the American Association of Pharmaceutical Scientists, Chicago, IL 2012. 